\title{
Algoritmos ABC em Environmental Stress Screening
}

Luis Gabriel Marques Reginato

\author{
DisSERTAÇÃO APRESENTADA \\ AO \\ Instituto De Matemática e EstatísticA \\ DA \\ Universidade DE SÃo PAUlo \\ PARA \\ OBTENÇÃO DO TÍTULO \\ $\mathrm{DE}$ \\ Mestre em CiênCIAS \\ Programa: Estatística \\ Orientador: Prof. Dr. Luís Gustavo Esteves \\ São Paulo, março de 2015
}




\section{Algoritmos ABC em \\ Environmental Stress Screening}

Esta versão da dissertação contém as correções e alterações sugeridas pela Comissão Julgadora durante a defesa da versão original do trabalho, realizada em 06/03/2015. Uma cópia da versão original está disponível no

Instituto de Matemática e Estatística da Universidade de São Paulo.

Comissão Julgadora:

- Prof. Dr. Luís Gustavo Esteves - IME-USP

- Prof. Dr. Sérgio Wechsler - IME-USP

- Prof. Dr. Rafael Izbicki - UFSCAR 
"O valor das coisas não está no tempo que elas duram, mas na intensidade com que acontecem. Por isso, existem momentos inesquecíveis, coisas inexplicáveis e pessoas incomparáveis."

Fernando Pessoa 
A minha mãe e minha vó. 


\section{Agradecimentos}

Agradeço

ao Professor Luís Gustavo Esteves, por me orientar e sempre me fazer acreditar mais em mim do que eu mesmo, pelos constantes ensinamentos e pela capacidade de transferir conhecimento sem subestimar os alunos;

aos Professores Sérgio Wechsler, do IME, e Rafael Izbicki, da UFSCAR, membros da banca de defesa, por seus valiosos comentários e sugestões que contribuíram para a melhoria deste trabalho;

novamente ao Professor Sérgio, e aos Professores Carlinhos e Cláudia Peixoto, do IME, e ao Professor Luís Eduardo Afonso, da FEA, pelas orientações em momentos distintos da minha trajetória;

aos irmãos jacobianos (Davi, Denis, Emerson, Fernando, Hommenig e Paulo), por sua amizade que começou na graduação e não terminará nesta vida;

a Rafael Bassi Stern e Renata Trevisan Brunelli, por terem cordial e pacientemente contribuído com discussões e insights dos mais variados;

aos demais colegas de graduação (IME e FEA) e mestrado, por terem propiciado momentos constantes de inspiração e aprendizado;

aos gestores, demais pares e equipe do Banco Itaú, por suportarem minha decisão de fazer o mestrado e pela convivência diária de muito respeito e cordialidade;

ao meu pai, Caio, meu irmão, João Paulo, e demais familiares por constantemente servirem de inspiração e referência;

à minha querida noiva Juliana Barby Simão, por querer me ajudar em todos os momentos e por sua capacidade de contribuir com todos os aspectos deste trabalho;

à minha vó Norma, pela imensa sapiência e por diversas vezes ter sido mais mãe e assim tantas vezes me ensinar mais do que me mimar;

à minha mãe, Sonia, pelas constantes orientação, paciência, capacidade de ouvir e ensinar, e por ser fundamental em todos os momentos importantes da minha vida. 


\section{Resumo}

Reginato, L. G. M. Algoritmos ABC em Environmental Stress Screening. 2015. 78 f. Dissertação de Mestrado - Instituto de Matemática e Estatística, Universidade de São Paulo, 2015.

É comum, em problemas de inferência bayesiana, deparar-se com uma distribuição a posteriori para o parâmetro de interesse, $\theta$, que seja intratável analitica ou computacionalmente. Como a priori é uma escolha do pesquisador, tal situação ocorre por conta da intratabilidade da função de verossimilhança. Por meio de algoritmos $\mathrm{ABC}$, é possível simular-se uma amostra da distribuição a posteriori, sem a utilização da função de verossimilhança.

Neste trabalho, aplica-se o ABC no contexto de Environmental Stress Screening - ESS. ESS é um procedimento de estresse, em um processo de produção industrial, que visa evitar que peças de qualidade inferior sejam utilizadas no produto final. A partir de uma abordagem bayesiana do ESS, depara-se com uma verossimilhança (e, consequentemente, uma posteriori) intratável para o vetor de parâmetros de interesse. Utiliza-se, então, o ABC para obtenção de uma amostra da posteriori e calcula-se o tempo ótimo de duração de um futuro procedimento de estresse a partir da simulação feita.

É também proposta uma generalização do problema de ESS para a situação em que existem $k$ tipos de peças no processo de produção. Quantifica-se o problema e, novamente, aplica-se um algoritmo ABC para a obtenção de uma simulação da posteriori, bem como calcula-se o tempo ótimo de duração de um futuro teste de estresse.

Palavras-chave: Algoritmos ABC, Environmental Stress Screening, inferência bayesiana, simulação. 


\section{Abstract}

Reginato, L. G. M. ABC algorithms in Environmental Stress Screening. 2015. 78 f. Dissertação de Mestrado - Instituto de Matemática e Estatística, Universidade de São Paulo, 2015.

In Bayesian inference problems, it is common to obtain a posterior distribution for the parameter of interest, $\theta$, which is analytically or computationally intractable. Since the priori is chosen by the researcher, this situation arises from the intractability of the likelihood function. Through ABC algorithms it is possible to simulate a sample from the posterior distribution, without the analytical use of the likelihood function.

In this work ABC is applied in the context of Environmental Stress Screening - ESS. ESS is a stress procedure, in an industrial production process, which aims to avoid low quality parts to be used in the final product. Under a Bayesian approach to ESS, an intractable likelihood (consequently, a posterior) is obtained for the paramater of interest. ABC is used to simulate a sample from the posterior and the optimal duration for a next stress procedure is calculated afterwards.

A generalization of the ESS is also proposed considering that there are $k$ types of parts in the production process. Again, ABC is used to simulate a sample from the posterior, and it is calculated the optimal duration for a next stress procedure.

Keywords: ABC algorithms, Environmental Stress Screening, Bayesian inference, simulation. 


\section{Sumário}

Lista de Figuras $\quad$ xi

Lista de Tabelas $\quad$ xiii

1 Introdução $\quad 1$

1.1 Organização do trabalho . . . . . . . . . . . . . . . . . . . . . 2

2 Algoritmos ABC $\quad 3$

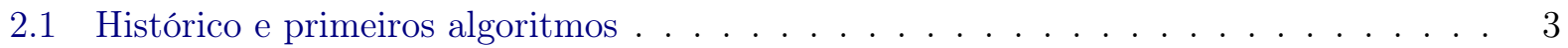

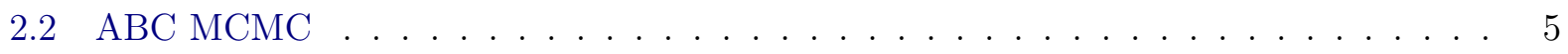

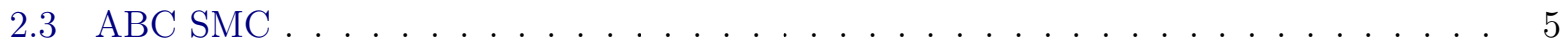

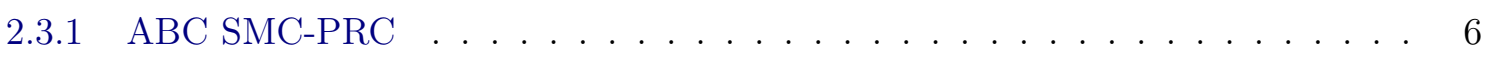

$2.3 .2 \mathrm{ABC} \mathrm{SMC}-\mathrm{PMC} \ldots \ldots \ldots \ldots \ldots \ldots$

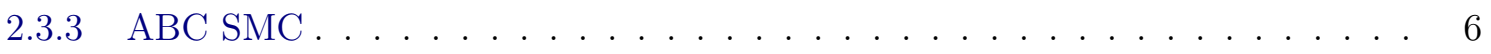

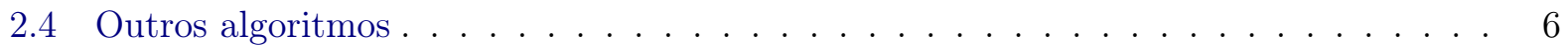

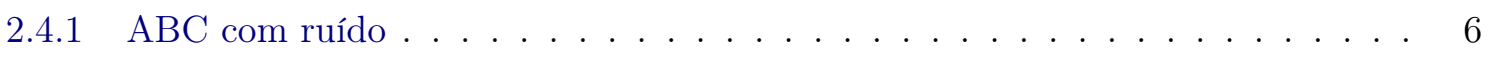

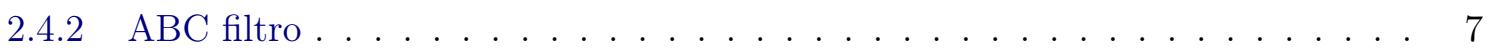

3 Environmental Stress Screening $\quad 9$

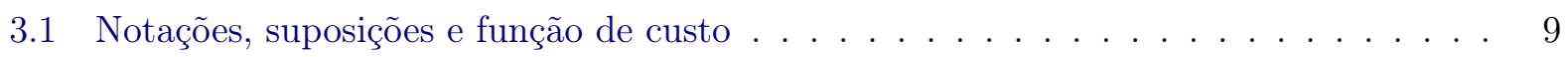

3.2 Modelagem bayesiana . . . . . . . . . . . . . . . . . . . . . . 10

4 Aplicação de ABC em ESS: caso de 2 tipos de peças $\quad 13$

4.1 Exemplos numéricos para $N$ pequeno . . . . . . . . . . . . . . . . . . 14

4.1 .1 Comparação com distribuições exatas . . . . . . . . . . . . . . . . . 14

4.1 .2 Algoritmo Rejeição $2 \ldots \ldots$. . . . . . . . . . . . . . . 16

4.2 Exemplos numéricos para $N$ grande $\ldots \ldots \ldots \ldots \ldots \ldots \ldots$

4.2 .1 Influência de $\varepsilon \ldots \ldots \ldots \ldots \ldots$

4.2 .2 Função de custo . . . . . . . . . . . . . . . . . . . . . . . . . . . . . . . 19

5 Aplicação para $k$ tipos de peças $\quad 23$

5.1 Modelagem bayesiana . . . . . . . . . . . . . . . . . . . 23

5.1 .1 Distribuição a priori dos parâmetros . . . . . . . . . . . . . . . 23

5.1 .2 Função de verossimilhança . . . . . . . . . . . . . . . . . . . . . 24

5.1 .3 Função de custo . . . . . . . . . . . . . . . . . . . . . 26

5.2 Aplicação do ABC: 3 tipos de peças . . . . . . . . . . . . . . . . . 27

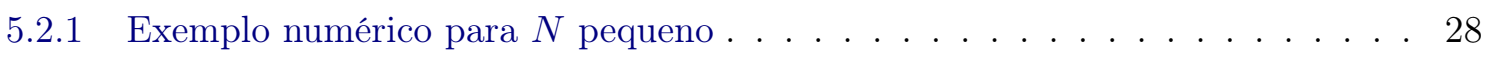


5.2 .2 Comparação com distribuições exatas . . . . . . . . . . . . . . . . . 28

5.2 .3 Algoritmo Rejeição $2 \ldots$. . . . . . . . . . . . . . . . . . . . . . . . 34

5.3 Exemplos numéricos para $N$ grande $\ldots \ldots \ldots \ldots \ldots \ldots$

5.3 .1 Função de custo . . . . . . . . . . . . . . . . . . . . . . 38

6 Conclusões $\quad 39$

6.1 Sugestões para pesquisas futuras . . . . . . . . . . . . . . . . . . . . 39

$\begin{array}{lll}\text { A Desenvolvimento da Expressão } 3.7 & 41\end{array}$

B Posterioris marginais de $\alpha, p, \lambda_{b}$ e $\lambda_{r}$ para 2 tipos de peças 43

B.1 Posteriori marginal de $\alpha \ldots \ldots \ldots \ldots \ldots \ldots \ldots \ldots$

B.2 Posteriori marginal de $p \ldots \ldots \ldots \ldots \ldots \ldots \ldots \ldots$

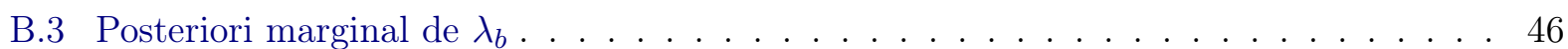

B.4 Posteriori marginal de $\lambda_{r} \ldots \ldots \ldots \ldots \ldots \ldots \ldots \ldots$

C Desenvolvimento da Expressão 5.5 49

D Posterioris marginais de $\alpha, p_{1}, p_{2}, p_{3}, \lambda_{1}, \lambda_{2}$ e $\lambda_{3}$ para 3 tipos de peças $\quad 51$

D.1 Posteriori marginal de $\alpha \ldots \ldots \ldots \ldots \ldots \ldots \ldots$

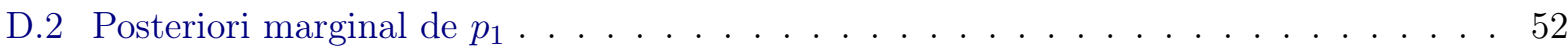

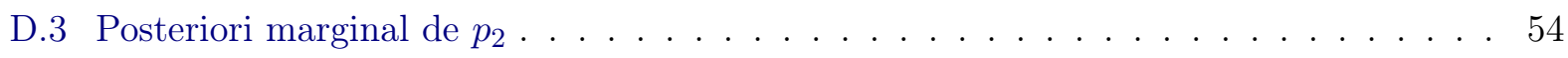

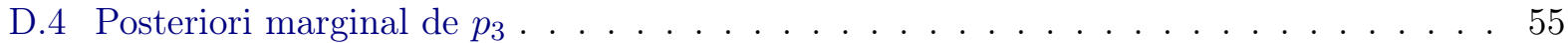

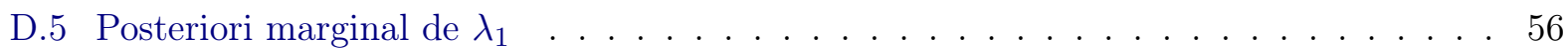

D.6 Posteriori marginal de $\lambda_{2} \ldots \ldots \ldots \ldots \ldots \ldots \ldots \ldots$

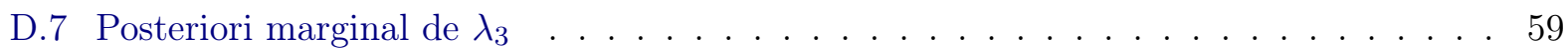

$\begin{array}{ll}\text { Referências Bibliográficas } & 61\end{array}$ 


\section{Lista de Figuras}

4.1 Histograma dos valores simulados de $\alpha, p, \lambda_{b}$ e $\lambda_{r} \ldots \ldots \ldots \ldots \ldots \ldots$

4.2 Densidade acumulada de $\alpha$ (em vermelho) e densidade simulada (em preto) . . . . . 15

4.3 Densidade acumulada de $p$ (em vermelho) e densidade simulada (em preto). . . . . . 15

4.4 Densidade acumulada de $\lambda_{b}$ (em vermelho) e densidade simulada (em preto). . . . . 16

4.5 Densidade acumulada de $\lambda_{r}$ (em vermelho) e densidade simulada (em preto). . . . . 16

4.6 Histograma da distância euclidiana entre os elementos de $\mathbb{T}_{10}^{4}$ e $(4,4,1,1) \quad \ldots$. . . 17

4.7 Densidades simuladas de $\alpha, p, \lambda_{b}$ e $\lambda_{r}$ para diferentes valores de $\varepsilon \ldots \ldots \ldots$

4.8 Função de risco a priori . . . . . . . . . . . . . . . . . . . . . . . . . . . 19

4.9 Histograma dos valores simulados de $\alpha, p, \lambda_{b}$ e $\lambda_{r} \ldots \ldots \ldots \ldots \ldots$

4.10 Densidades acumuladas a posteriori de $\alpha, p, \lambda_{b}$ e $\lambda_{r}$ simuladas a partir do algoritmo Rejeição 2 para diferentes valores de $\varepsilon \ldots \ldots \ldots \ldots . \ldots \ldots . \ldots \ldots$

4.11 Função de risco a posteriori . . . . . . . . . . . . . . . . . . . . . . 21

5.1 Histograma dos valores simulados de $\alpha, p_{1}, p_{2}, p_{3}, \lambda_{1}, \lambda_{2}$ e $\lambda_{3} \ldots \ldots \ldots \ldots$

5.2 Densidade acumulada de $\alpha$ (em vermelho) e densidade simulada (em preto) . . . . . 30

5.3 Densidade acumulada de $p_{1}$ (em vermelho) e densidade simulada (em preto). . . . . . 30

5.4 Densidade acumulada de $p_{2}$ (em vermelho) e densidade simulada (em preto). . . . . . 31

5.5 Densidade acumulada de $p_{3}$ (em vermelho) e densidade simulada (em preto). . . . . . 32

5.6 Densidade acumulada de $\lambda_{1}$ (em vermelho) e densidade simulada (em preto). . . . . 32

5.7 Densidade acumulada de $\lambda_{2}$ (em vermelho) e densidade simulada (em preto). . . . . 33

5.8 Densidade acumulada de $\lambda_{3}$ (em vermelho) e densidade simulada (em preto). . . . . 34

5.9 Histograma da distância euclidiana entre os elementos de $\mathbb{T}_{8}^{5}$ e $(3,1,2,1,1) \ldots \ldots$

5.10 Densidades simuladas de $\alpha, p_{1}, p_{2}, p_{3}, \lambda_{1}, \lambda_{2}$ e $\lambda_{3}$ para diferentes valores de $\varepsilon \ldots 35$

5.11 Função de risco a priori . . . . . . . . . . . . . . . . . . . . 36

5.12 Histograma dos valores simulados de $\alpha, p_{1}, p_{2}, p_{3}, \lambda_{1}, \lambda_{2}$ e $\lambda_{3} \ldots \ldots \ldots \ldots$

5.13 Função de risco a posteriori . . . . . . . . . . . . . . . . . . 38 


\section{Lista de Tabelas}

3.1 Quantidade de parcelas na expressão 3.7 dados valores de $x, y, z$ e $m$. . . . . . 12 


\section{Capítulo 1}

\section{Introdução}

Na condução de uma análise estatística, como em problemas de testes de hipótese e estimação, pode-se optar por alguma abordagem, dentre as quais as mais comuns são a clássica (ou frequentista) e bayesiana (ou subjetivista). Na abordagem clássica, o parâmetro de interesse, $\theta$, considerado fixo e desconhecido, é usualmente estimado encontrando-se $\widehat{\theta}$ que minimiza (dentre o universo de valores possíveis de $\theta$ ) alguma função de perda envolvendo $\theta$ e os dados. Tal minimização envolve apenas a distribuição dos dados condicional a $\theta$.

Do ponto de vista bayesiano, os parâmetros - assim como os dados - são considerados variáveis aleatórias. Desta forma, o exercício de inferência baseia-se na distribuição de probabilidade dos parâmetros após a observação dos dados, chamada de distribuição a posteriori (ou simplesmente posteriori). A obtenção da posteriori se baseia no famoso teorema de Bayes, pelo qual $f(\theta \mid x) \propto$ $L(\theta \mid x) f(\theta)$. É necessário, portanto, que se estabeleça uma distribuição a priori (ou simplesmente priori) para $\theta$, dada por $f(\theta)$, bem como que se defina a função de verossimilhança de $\theta$ fixados os valores observados, dada por $L(\theta \mid x)$.

A priori representa numericamente a opinião do pesquisador a respeito de $\theta$, e assumimos que seu tratamento analítico é possível. Porém, o mesmo não se pode afirmar da função de verossimilhança: Marin et al. (2011) afirmam que a função de verossimilhança pode ser intratável por razões matemáticas (não existe de forma fechada como uma função de $\theta$ ) ou por razões computacionais (seu cálculo é muito custoso ou até inviável). Desta forma, o avanço recente dos métodos computacionais de simulação levou ao desenvolvimento de técnicas para realizar inferência estatística em problemas nos quais a posteriori (ou a verossimilhança) não é analiticamente tratável, dentre as quais destacam-se os algoritmos ABC (Approximate Bayesian computation ou computação Bayesiana aproximada).

Segundo Bonassi (2013), a ideia chave do método ABC é simular dados da posteriori substituindo o cálculo da verossimilhança por algumas etapas de simulação do modelo. O mesmo autor afirma que a técnica permite grande flexibilidade podendo ser aplicada a qualquer problema do qual seja possível simular o modelo de interesse.

Neste trabalho, iremos aplicar o ABC no contexto de ESS: Environmental Stress Screening. ESS é um procedimento frequentemente utilizado em indústrias visando detectar peças defeituosas em um processo de produção. Tal procedimento evita, segundo Reddy e Dietrich (1994), que tais defeitos causem falhas precoces nas peças e no produto final, que resultam em custos significativos tanto para o produtor quanto para o consumidor final.

Conforme Barlow et al. (1990), uma questão relevante no contexto do ESS é o tempo de duração do procedimento. Em Barlow et al. (1994), os autores apresentam uma quantificação do procedi- 
mento, a partir de uma abordagem bayesiana, para o caso em que há 2 tipos de peças, boas ou ruins. Veremos que, dependendo de certos fatores (como o número de peças envolvidas no procedimento), a posteriori resultante mostra-se intratável analiticamente, motivando a aplicação do ABC e permitindo-se calcular o tempo ótimo de duração do estresse.

Por fim, iremos propor uma quantificação do procedimento para um caso mais geral, no qual há $k$ tipos de peças, que se diferenciam de acordo com seu nível de qualidade. Novamente obteremos uma posteriori intratável e, a partir do ABC, obtemos uma amostra da posteriori e calculamos o tempo ótimo de duração do estresse.

\subsection{Organização do trabalho}

O trabalho está dividido em 6 capítulos, mais o apêndice. No capítulo 2, apresentamos as definições de algoritmos ABC e alguns exemplos. Conceitos de Environmental Stress Screening (ESS) são introduzidos no capítulo 3, no qual também quantificamos o problema motivando o uso do ABC para sua resolução.

No capítulo 4, resolvemos o problema tradicional - onde as peças do processo de produção podem ser qualificadas como de boa ou de má qualidade - do ponto de vista teórico, bem como damos um exemplo prático de aplicação com dados numéricos utilizando o ABC. Uma extensão do problema para $k$ tipos de falha - ou seja, as peças podem ter vários níveis de qualidade - é apresentada no capítulo 5, bem como sua resolução teórica e um exemplo prático para o caso $k=3$. Por fim, apresentamos as conclusões no capítulo 6 . 


\section{Capítulo 2}

\section{Algoritmos ABC}

\subsection{Histórico e primeiros algoritmos}

Enquanto metodologia, o ABC (approximate Bayesian computation - ou computação Bayesiana aproximada) é mencionado desde o início da década de 1980, quando Rubin (1984) discutia um argumento pedagógico e filosófico pelo qual "estatística Bayesiana e métodos de Monte Carlo são idealmente apropriados à tarefa de passar vários modelos por um conjunto de dados" (Marin et al, 2011).

Fundamentalmente, o ABC consiste em simular observações da posteriori sem necessidade do uso analítico da função de verossimilhança. Portanto, sua utilidade se evidencia em problemas de inferência bayesiana nos quais a verossimilhança não é tratável analiticamente ou mesmo computacionalmente.

O algoritmo Rejeição 1, introduzido em Rubin (1984) e apresentado a seguir como em Marin et al. (2011), é o algoritmo mais básico em que tal ideia é praticada, conforme Campos e Wechsler (2012). A partir da priori $f(\theta)$ escolhida e dos dados observados $x$, sorteia-se um candidato $\theta^{\prime}$ (de maneira geral, no passo inicial deste e de algoritmos similares, $\theta^{\prime}$ recebe o nome de "candidato", uma vez que precisaremos decidir posteriormente se o aceitamos de fato como um valor observado da posteriori de $\theta)$.

Sorteia-se, então, uma observação $y$ a partir de $f\left(y \mid \theta^{\prime}\right)$. É no passo seguinte que o conceito de rejeição se aplica. Os valores observados $x$ servirão de referência para a decisão de aceitar ou não o candidato $\theta^{\prime}$. Caso obtenha-se $y=x$, aceita-se $\theta^{\prime}$ como um valor observado da posteriori $f(\theta \mid x)$. Caso contrário, rejeita-se $\theta^{\prime}$.

O procedimento é repetido até que se obtenha $n$ valores (onde $n$ é previamente definido pelo pesquisador) de $\theta^{\prime}$ aceitos, conforme esquematizado a seguir.

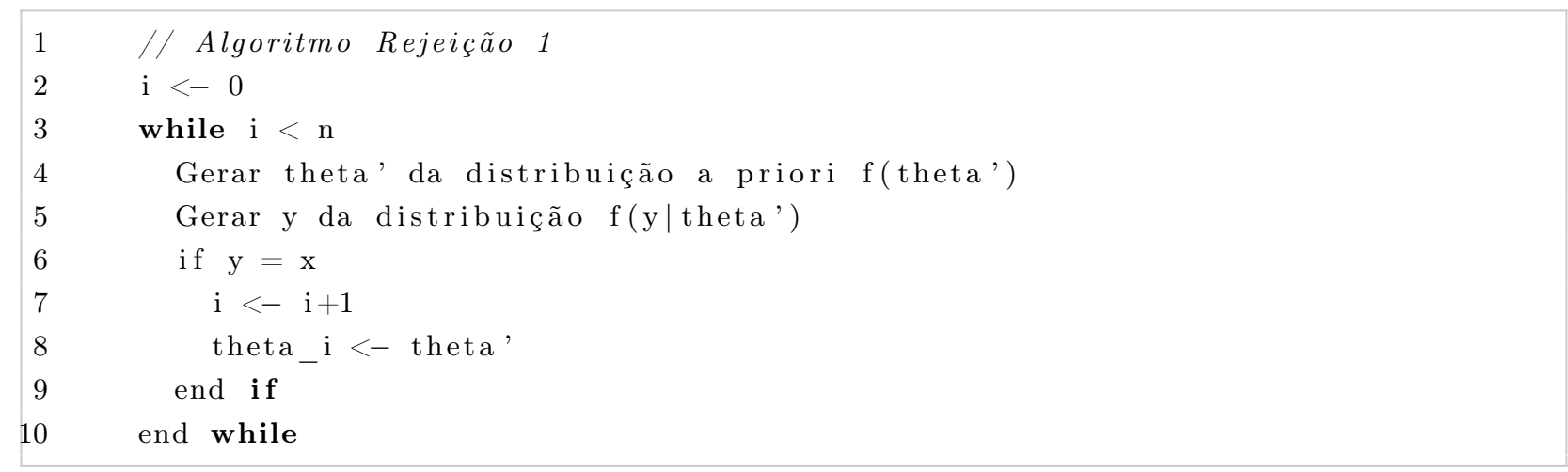

Neste algoritmo, é necessário supor que $x \in \mathbb{D} \subseteq \mathbb{R}^{n}$, onde $\mathbb{D}$ é um conjunto finito ou enumerável, limitando sua utilização a problemas dessa natureza. Nos casos em que $|D|$ é infinito ou muito 
grande, pode-se demorar muito para obter a condição $y=x$, tornando o algoritmo ineficiente.

O resultado final do algoritmo Rejeição 1 é, portanto, um vetor $\left(\theta_{1}, \ldots, \theta_{n}\right)$, onde seus elementos são independentes e identicamente distribuídos conforme a posteriori de $\theta$, pois, $\forall i \in\{1, \ldots, n\}$,

$$
f\left(\theta_{i}\right) \propto f(\theta) f(y \mid \theta) \mathbb{1}_{(y=x)}=f(\theta) f(x \mid \theta) \propto f(\theta \mid x),
$$

onde $\mathbb{1}_{(a=b)}=1$, se $a=b$, e $\mathbb{1}_{(a=b)}=0$, caso contrário.

De maneira geral, os trabalhos que formalizaram o método e o próprio nome $\mathrm{ABC}$ abordaram problemas no campo de genética populacional. Em Tavaré et al. (1997), os autores apresentam um algoritmo no qual, a cada etapa, é simulado um candidato $\theta^{\prime}$ proveniente da priori de $\theta$. Neste algoritmo, aceita-se $\theta^{\prime}$ se, e somente se, $\mathbb{P}\left(S=s \mid \theta^{\prime}\right)>c U$, onde $S$ é uma estatística dos dados e $s$ é o seu valor observado, $U \sim U[0,1]$ e $c$ é uma constante satisfazendo $c \geq \max _{\theta} \mathbb{P}(S=s \mid \theta)$.

O algoritmo de Tavaré et al. (1997) se limita a casos simples em que $\mathbb{P}(S=s \mid \theta)$ pode ser calculada e maximizada em $\theta$. Pritchard et al. (1999) introduziram um algoritmo de rejeição em que, após simular-se o candidato $\theta^{\prime}$ a partir da priori, simula-se um valor $y$ de $f\left(y \mid \theta^{\prime}\right)$ e aceita-se $\theta^{\prime}$ caso $d(S(y), S(x)) \leq \varepsilon$, onde $d$ é alguma função de comparação (ou distância) e $\varepsilon$ é um valor arbitrário que determina o limite aceitável da distância entre a estatística $S(y)$, obtida a partir da simulação, e $S(x)$, obtida por meio dos dados observados. Produziu-se, assim, o primeiro algoritmo ABC genuíno (Marin et al, 2011).

Este algoritmo, denominado Rejeição 2, é uma extensão do algoritmo Rejeição 1, podendo ser aplicado em situações nas quais o espaço amostral é contínuo, ou seja, $x \in \mathbb{D} \subseteq \mathbb{R}^{n}$.

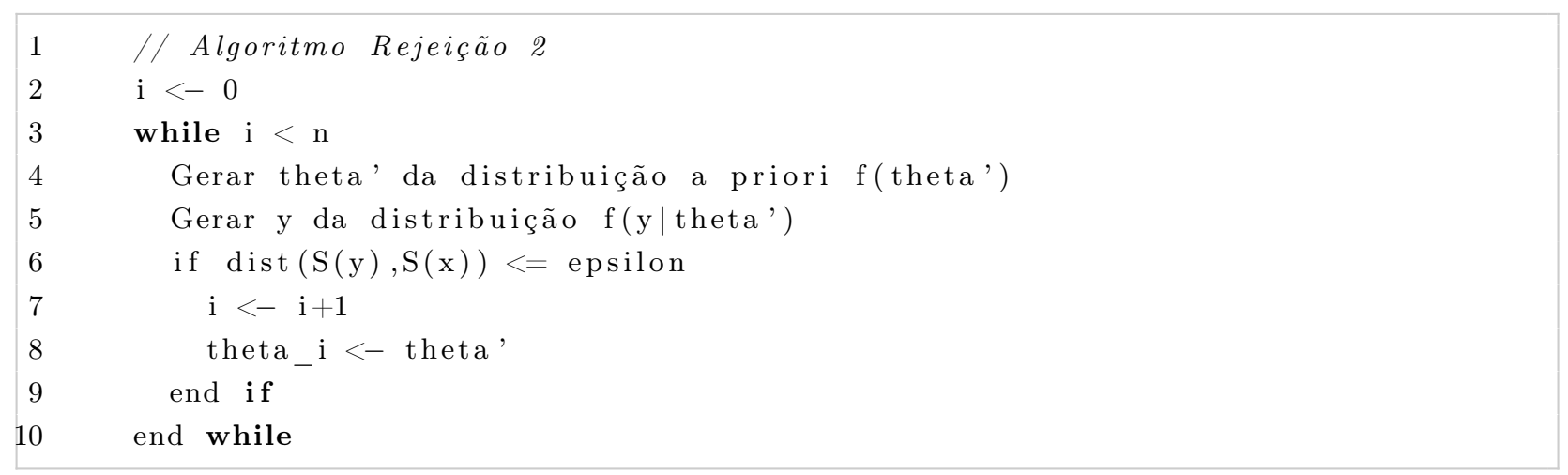

Assim como no algoritmo Rejeição 1, resulta do algoritmo Rejeição 2 um vetor $\left(\theta_{1}, \ldots, \theta_{n}\right)$. Conforme Marin et al. (2011), o fundamento por trás do ABC é que usando uma estatística representativa $S$ associada a um pequeno limite de tolerância $\varepsilon$ deve-se obter uma boa aproximação da posteriori, uma vez que, $\forall i \in\{1, \ldots, n\}$,

$$
f_{\varepsilon}\left(\theta_{i}\right) \propto \int_{\mathbb{D}} f_{\varepsilon}\left(\theta_{i}, y\right) \mathbb{1}_{y \in A_{\varepsilon, x}} \mathrm{~d} y=\int_{\mathbb{D}} f(\theta) f(y \mid \theta) \mathbb{1}_{y \in A_{\varepsilon, x}} \mathrm{~d} y \approx f(\theta) f(x \mid \theta) \propto f(\theta \mid x),
$$

onde $A_{\varepsilon, x}=\{y \in \mathbb{D} \mid d(S(y), S(x)) \leq \varepsilon\}$.

Bonassi (2013) argumenta que, embora o ABC possua grande flexibilidade, um potencial problema seriam as altas taxas de rejeição dos algoritmos, decorrentes de: (i) a necessidade de $\varepsilon$ ser pequeno o suficiente para que a posteriori simulada seja uma boa aproximação de $p(\theta \mid x)$, e (ii) 
o fato de que a posteriori $p(\theta \mid x)$ pode estar concentrada em regiões do espaço paramétrico completamente distintas da priori. Assim, modificações nos algoritmos básicos ABC foram surgindo, incluindo ajustes por meio de técnicas de regressão (Beaumont et al, 2002) e esquemas de amostragem automáticos (Marjoram et al, 2003).

\section{$2.2 \quad$ ABC MCMC}

Existem situações em que se torna necessária a utilização de algoritmos ABC mais sofisticados. Por exemplo, há casos em que a simulação a partir da priori $f(\theta)$ pode ser ineficiente e levar a valores simulados em regiões de baixa probabilidade a posteriori, uma vez que a escolha da priori não leva em conta os dados (Marin et al, 2011). Uma maneira de lidar com este problema é vista em Marjoram et al. (2003). Os autores propõem o algoritmo $A B C-M C M C$, no qual se introduz uma cadeia de Markov associada a um núcleo Markoviano para convergência dos valores simulados a regiões mais esperadas da densidade a posteriori.

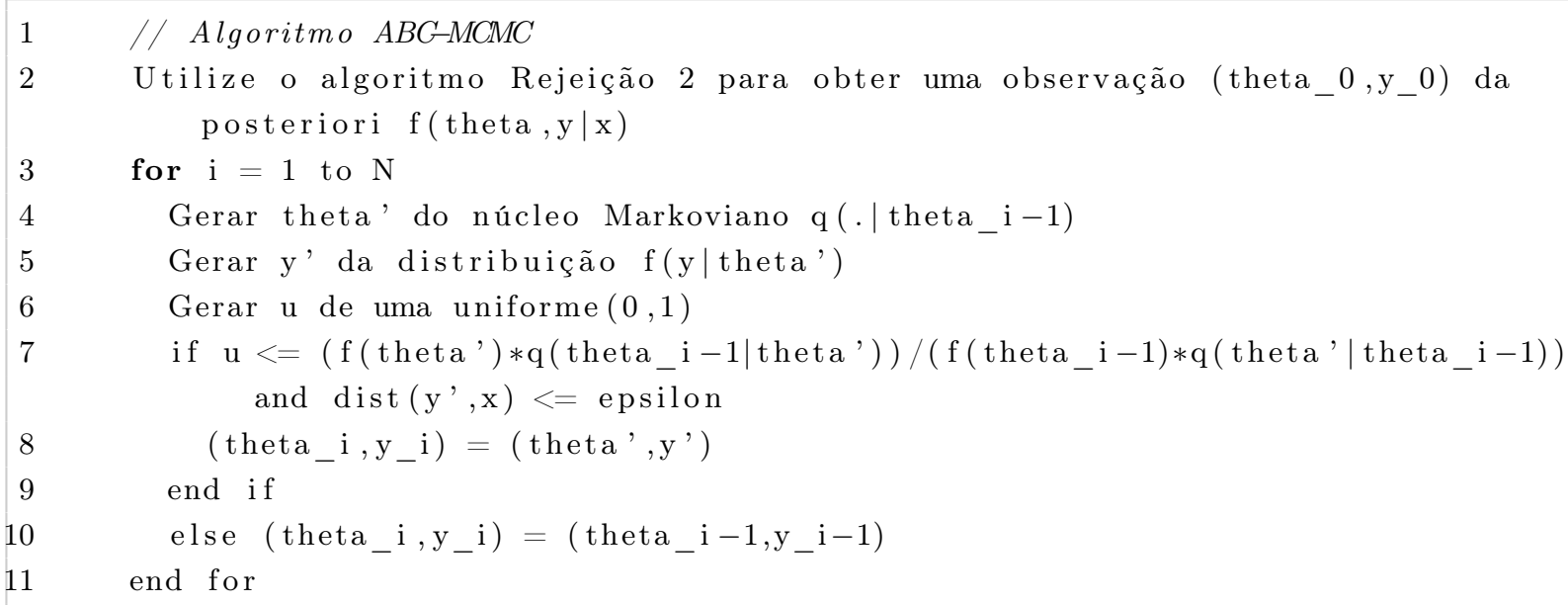

Note que continua sendo necessária a definição dos valores arbitrários $\varepsilon$ e da função de distância d. Na linha 7 do algoritmo tem-se a aplicação do núcleo markoviano, no qual aceita-se a mudança de estado na cadeia de Markov no caso em que $u \leq \frac{f\left(\theta^{\prime}\right) * q\left(\theta_{i-1} \mid \theta^{\prime}\right)}{f\left(\theta_{i-1}\right) * q\left(\theta^{\prime} \mid \theta_{i-1}\right)}$, onde $u \sim U[0,1]$. Ou seja, o algoritmo garante que estamos sempre nos aproximando de regiões mais prováveis da posteriori.

\section{$2.3 \quad$ ABC SMC}

No algoritmo apresentado em 2.2, trabalhamos com candidatos únicos $\theta^{\prime}$ a cada iteração. Uma alternativa a esta abordagem é trabalhar com um pool de candidatos (chamados de partículas) simultaneamente. Conforme o trabalho de Turner e Van Zandt (2012), as partículas são perturbadas e filtradas a cada passo do algoritmo, aproximando o pool de partículas cada vez mais de uma observação da posteriori desejada. A este tipo de abordagem dá-se o nome de Sequential Monte Carlo ABC, ou ABC SMC. Nas seções a seguir, apresentamos, brevemente, alguns exemplos de algoritmos que utilizam esta técnica. 


\subsubsection{ABC SMC-PRC}

O trabalho de Sisson et al. (2007) desenvolveu um algoritmo de controle de rejeição parcial (partial rejection control - PRC), sendo o primeiro algoritmo ABC a utilizar um filtro de partícula (Turner e Van Zandt, 2012).

Neste algoritmo, é requerido um núcleo de transição progressivo $\left(q_{p}\left(\cdot \mid \theta^{\prime}\right)\right)$ e um outro regressivo $\left(q_{r}\left(\cdot \mid \theta^{\prime \prime}\right)\right)$. Usa-se o núcleo progressivo para passar de $\theta^{\prime}$ a $\theta^{\prime \prime}$ e, a partir de $\theta^{\prime \prime}$, simulamos $X$ e comparamos com os dados por meio da função de distância. Aceitamos $\theta^{\prime \prime}$ se a distância for menor que algum $\varepsilon$, e atribuímos um peso $w$ a $\theta^{\prime \prime}$ por meio da relação $w=\frac{f\left(\theta^{\prime \prime}\right) q_{r}\left(\theta^{\prime} \mid \theta^{\prime \prime}\right)}{f\left(\theta^{\prime}\right) q_{p}\left(\theta^{\prime \prime} \mid \theta^{\prime}\right)}$. Repete-se o processo até obtermos $N$ partículas novas satisfazendo a relação de distância.

O processo acima é similar ao algoritmo apresentado em 2.2. Porém, repetimos o processo diversas vezes, e a cada iteração sorteamos as novas partículas por meio do peso atribuído na iteração anterior. Isto garante a obtenção de amostras distribuídas nas regiões mais prováveis da posteriori de $\theta$, evitando que a cadeia fique presa em regiões de baixa probabilidade caso utilize-se o algoritmo apresentado em 2.2. A eficiência da simulação irá depender fortemente da escolha das funções de transição bem como da priori $f$.

\subsubsection{ABC SMC-PMC}

O algoritmo ABC população Monte Carlo (Monte Carlo population - PMC) utiliza um esquema de ponderação diferente do SMC-PRC apresentado na seção anterior. No SMC-PMC, é necessário apenas um núcleo gaussiano de transição $q\left(\cdot \mid \theta^{\prime}\right)$, que depende da variância das partículas observadas na iteração anterior. Tal abordagem permite melhorar a eficiência do algoritmo e diminuir o tempo para obtenção de amostras da posteriori. Detalhamentos deste algoritmo podem ser encontrados em Beaumont et al. (2009) e Beaumont (2010).

\subsubsection{ABC SMC}

Nos casos em que $\theta$ não pode ter suporte ilimitado (por exemplo, quando $\theta$ é a probabilidade de referência em uma distribuição binomial), é introduzido no trabalho de Toni et al. (2009) um algoritmo similar ao SMC-PMC, com a diferença que o núcleo $q\left(\cdot \mid \theta^{\prime}\right)$ não é necessariamente gaussiano.

\subsection{Outros algoritmos}

Outros tipos de algoritmos de simulação aproximada, que não fazem uso da distribuição a posteriori e que, portanto, se encaixam na definição de algoritmos $\mathrm{ABC}$, foram propostos e alguns exemplos estão apresentados nas subseções a seguir.

\subsubsection{ABC com ruído}

O trabalho de Wilkinson (2009) apresenta um algoritmo que substitui o erro de aproximação do algoritmo $\mathrm{ABC}$ por uma inferência exata a partir de uma aproximação controlada da função objetivo, basicamente uma convolução do objetivo com um núcleo arbitrário. Maiores detalhes deste algoritmo podem ser encontrados nos trabalhos de Marin et al. (2011) e Fearnhead e Prangle (2012). 


\subsubsection{ABC filtro}

O trabalho de Jasra et al. (2012) introduz um algoritmo ABC com filtro via modelos de Markov ocultos (ou hidden Markov models - HMM). Os autores utilizam um algoritmo sequencial de Monte Carlo para amostrar e estimar a posteriori a partir de uma aproximação ABC da densidade alvo. 


\section{Capítulo 3}

\section{Environmental Stress Screening}

O procedimento de Environmental Stress Screening (ESS) consiste em submeter determinadas peças a um processo de estresse - geralmente, pela variação extrema de temperatura ou outros fatores - visando antecipar falhas dessas unidades, permitindo identificação e eliminação das peças de qualidade inferior e evitando assim sua utilização no produto final. O benefício deste método é claro, uma vez que o custo de se estressar as peças é inferior àquele decorrente de falhas que venham a ocorrer após a confecção do equipamento final, conforme apontado em Barlow et al. (1994).

O ESS vem sendo usado com sucesso na indústria para combater a mortalidade precoce das peças finais (Yang 2002). Segundo Barlow et al. (1994), um planejamento de ESS deve ser realizado levando em conta três aspectos principais: o tipo de estresse, a intensidade do estresse e a duração do procedimento.

Neste trabalho, abordaremos mais detalhadamente a questão da duração do procedimento. Utilizaremos, para este fim, uma quantificação do processo introduzida em Perlstein et al. (1987) e, posteriormente, abordada do ponto de vista bayesiano nos trabalhos de Barlow et al. (1990) e Barlow et al. (1994).

Por meio da análise bayesiana sugerida em Barlow et al. (1994), é possível obter informações a respeito da proporção de peças de cada nível de qualidade, bem como da taxa de falha de cada tipo de peça e, principalmente, inferir a duração ótima do tempo de duração do teste de estresse para futuros experimentos. Tal inferência é importante, uma vez que há uma série de custos envolvidos no processo de estressar as peças e, portanto, o tempo ótimo de duração deve ser aquele que minimiza os custos do processo.

\subsection{Notações, suposições e função de custo}

Iremos trabalhar, neste capítulo, com as suposições apresentadas em Barlow et al. (1994). Primeiramente, os autores consideram um lote com $N$ peças, dentre as quais uma proporção $p$ possui nível de qualidade ruim, que posteriormente levariam a uma falha do equipamento caso fossem utilizadas. As peças de qualidade ruim não podem ser detectadas por um processo de controle de qualidade tradicional, pois possuem a mesma aparência daquelas de qualidade boa. Esta é a mesma configuração do problema apresentada em Perlstein et al. (1987). As peças serão submetidas a um processo de estresse, da seguinte forma:

1. Amostragem de um lote de peças produzidas;

2. Submissão das peças amostradas a um procedimento de estresse, de intensidade e tempo pré-definidos; 
3. Após o final do teste, verificação de quais peças sobreviveram ao teste e quais não sobreviveram;

4. Verificação da qualidade das peças que não sobreviveram.

Os autores consideram que a intensidade do estresse pode ser interpretada como um fator constante de aceleração do tempo, dado por $l$, sendo $l>0$. Na prática, isto significa que a taxa de falha das peças de qualidade ruim, em condições normais, é dada por $\lambda_{r}^{\prime}$, e sob condições de estresse, dada por $\lambda_{r}=l \lambda_{r}^{\prime}$. Por sua vez, a taxa de falha das peças de qualidade boa, em condições normais, é dada por $\lambda_{b}^{\prime}$, e sob condições de estresse, dada por $\lambda_{b}=l \lambda_{b}^{\prime}$. Pressupõe-se que $\lambda_{r}^{\prime}>\lambda_{b}^{\prime}$ $\mathrm{e}$, portanto, $\lambda_{r}>\lambda_{b}$.

Conforme citado anteriormente, existem custos envolvidos no processo de estressar as peças. Os custos são tanto operacionais (custos do estresse em si) quanto de "decisões erradas". Denota-se por $c_{1}$ o custo de se estressar uma peça de nível ruim e esta sobreviver ao estresse, e por $c_{2}$ o custo de se estressar uma peça de nível bom e esta falhar. Portanto, $c_{1}$ e $c_{2}$ representam, de certa maneira, os custos de "se tomar decisões incoerentes" no processo. Para os custos operacionais, $c_{3}$ representa o custo de se estressar uma peça qualquer e esta falhar durante o processo e $c_{4}$ é o custo de se estressar uma peça qualquer e esta sobreviver.

Em geral, tem-se um prejuízo maior ao liberar peças de nível ruim (pois estas farão parte do produto final) do que ao falhar uma peça de nível bom, de tal maneira que supõe-se $c_{1}>c_{2}$. Em alguns casos, pode-se considerar $c_{2}=0$, quando não há diferença entre as peças de nível ruim e aquelas de nível bom que, porém, falham ao estresse. Também é assumido, por simplicidade, que os custos $c_{3}$ e $c_{4}$ não dependem da qualidade da peça nem do tempo de duração do processo $t$.

Tendo em vista o objetivo de determinar o tempo ótimo de duração de um estresse, Barlow et al. (1994) obtêm a expressão 3.1, que representa o custo do experimento por peça, dados os valores de $p, \lambda_{b}$ e $\lambda_{r}$, bem como do tempo de duração $t$.

$$
C\left(t, p, \lambda_{b}, \lambda_{r}\right)=p\left[\left(1-e^{-\lambda_{r} t}\right) c_{3}+e^{-\lambda_{r} t}\left(c_{1}+c_{4}\right)\right]+(1-p)\left[\left(1-e^{-\lambda_{b} t}\right)\left(c_{2}+c_{3}\right)+e^{-\lambda_{b} t} c_{4}\right]
$$

Deve-se salientar que a intensidade do estresse está diretamente associada aos níveis das taxas de falha $\lambda_{b}$ e $\lambda_{r}$.

\subsection{Modelagem bayesiana}

Seguindo a modelagem apresentada em Barlow et al. (1994), é assumido que, a priori, $p$ segue uma distribuição Beta de parâmetros $(a, b)$, onde $a>0$ e $b>0$. Para as taxas de falha, $\lambda_{b}$ possui uma distribuição exponencial de parâmetro $\theta, \operatorname{com} \theta>0$, e $\lambda_{r}$, dado $\lambda_{b}$, possui uma distribuição exponencial de parâmetro $\tau(\tau>0)$, deslocada por $\lambda_{b}$. Conforme apontado pelos autores, o uso da distribuição exponencial para as taxas de falha pressupõe que as peças, após o processo de estresse, não envelhecem, mantendo constantes suas taxas de falha.

Assim:

$$
f(p)=\frac{\Gamma(a+b)}{\Gamma(a) \Gamma(b)} p^{a-1}(1-p)^{b-1} \mathbb{1}_{(0 \leq p \leq 1)}
$$

e

$$
f\left(\lambda_{b}, \lambda_{r}\right)=\theta \tau e^{-\lambda_{b}(\theta-\tau)} e^{-\lambda_{r} \tau} \mathbb{1}_{\left(\lambda_{r}>\lambda_{b}>0\right)}
$$


Supondo que as taxas $\lambda_{b}$ e $\lambda_{r}$ são independentes de $p$, chega-se a:

$$
f\left(p, \lambda_{b}, \lambda_{r}\right)=f(p) f\left(\lambda_{b}, \lambda_{r}\right)=\theta \tau \frac{\Gamma(a+b)}{\Gamma(a) \Gamma(b)} p^{a-1}(1-p)^{b-1} e^{-\lambda_{b}(\theta-\tau)} e^{-\lambda_{r} \tau} \mathbb{1}_{(0 \leq p \leq 1)} \mathbb{1}_{\left(\lambda_{r}>\lambda_{b}>0\right)}
$$

Iremos incorporar os dados obtidos no procedimento de estresse e faremos a análise sob a ótica da inferência Bayesiana. Novamente, utilizamos as notações apresentadas em Barlow et al. (1994) para quantificação dos dados observados. Assim, define-se:

- $x$ : quantidade de peças que falharam durante o estresse e que foram classificadas como de qualidade ruim após a autópsia,

- $y$ : quantidade de peças que falharam durante o estresse e que foram classificadas como de qualidade boa após a autópsia,

- z: quantidade de peças que falharam durante o estresse e que não foram classificadas após a autópsia (isto é, não foi possível verificar o nível de qualidade delas),

- $m$ : quantidade de peças que sobreviveram ao estresse, sendo $m=N-x-y-z$.

Considera-se, portanto, a possibilidade de que não seja possível verificar a qualidade de algumas peças que falham no processo. A probabilidade de não ser possível verificar o nível de qualidade de alguma peça que falhou no estresse é assumida igual para todas as peças, independentemente da qualidade da peça, e dada por $\alpha(0<\alpha<1)$, que será assumida, a priori, como uma uniforme contínua em $[0,1]$.

Para cada peça do lote associa-se as seguintes probabilidades:

- $\mathbb{P}($ falhar no estresse e ser qualificada como de nível ruim $)=(1-\alpha) p\left(1-e^{-\lambda_{r} t}\right)$

- $\mathbb{P}($ falhar no estresse e ser qualificada como de nível bom $)=(1-\alpha)(1-p)\left(1-e^{-\lambda_{b} t}\right)$

- $\mathbb{P}($ falhar no estresse e não ser possível ver seu nível $)=\alpha p\left(1-e^{-\lambda_{r} t}\right)+\alpha(1-p)\left(1-e^{-\lambda_{b} t}\right)$

- $\mathbb{P}($ sobreviver ao estresse $)=p e^{-\lambda_{r} t}+(1-p) e^{-\lambda_{b} t}$

Assim, a verossimilhança gerada pelos dados é dada por:

$$
\begin{array}{r}
L\left(\alpha, p, \lambda_{b}, \lambda_{r} \mid x, y, z, m\right) \propto\left[(1-\alpha) p\left(1-e^{-\lambda_{r} t}\right)\right]^{x}\left[(1-\alpha)(1-p)\left(1-e^{-\lambda_{b} t}\right)\right]^{y} \\
{\left[\alpha p\left(1-e^{-\lambda_{r} t}\right)+\alpha(1-p)\left(1-e^{-\lambda_{b} t}\right)\right]^{z}\left[p e^{-\lambda_{r} t}+(1-p) e^{-\lambda_{b} t}\right]^{m}}
\end{array}
$$

Pode-se provar que o modelo proposto não apresenta a propriedade de identificabilidade, ou seja, não vale que, se $L\left(\alpha_{1}, p_{1}, \lambda_{b 1}, \lambda_{r 1} \mid x, y, z, m\right)=L\left(\alpha_{2}, p_{2}, \lambda_{b 2}, \lambda_{r 2} \mid x, y, z, m\right), \forall(x, y, z, m) \in \mathbb{N}^{4}$, tais que $x+y+z+m=N$, então $\left(\alpha_{1}, p_{1}, \lambda_{b 1}, \lambda_{r 1}\right)=\left(\alpha_{2}, p_{2}, \lambda_{b 2}, \lambda_{r 2}\right)$. 


\begin{tabular}{c|c|c|c|c}
\hline$x$ & $y$ & $z$ & $m$ & parcelas \\
\hline 1 & 1 & 1 & 1 & $\mathbf{3 2}$ \\
2 & 2 & 2 & 2 & $\mathbf{2 7 0}$ \\
5 & 5 & 5 & 5 & $\mathbf{1 3 . 3 9 2}$ \\
10 & 10 & 10 & 10 & $\mathbf{1 . 5 0 9 . 3 5 4}$ \\
20 & 20 & 20 & 20 & $\mathbf{9 . 7 1 4 . 7 5 1 . 9 5 6}$ \\
\hline
\end{tabular}

Tabela 3.1: Quantidade de parcelas na expressão 3.7 dados valores de $x, y, z$ e $m$.

A expressão da densidade conjunta a posteriori é dada por:

$$
f\left(\alpha, p, \lambda_{b}, \lambda_{r} \mid x, y, z, m\right)=\mathbb{K} f\left(p, \lambda_{b}, \lambda_{r}\right) L\left(\alpha, p, \lambda_{b}, \lambda_{r} \mid x, y, z, m\right)
$$

Na expressão anterior, $\mathbb{K}$ é a constante de normalização da densidade. Após o desenvolvimento, chega-se à seguinte expressão:

$$
\begin{array}{r}
f\left(\alpha, p, \lambda_{b}, \lambda_{r} \mid x, y, z, m\right) \propto \\
\propto \alpha^{z}(1-\alpha)^{x+y} \sum_{i=0}^{x} \sum_{j=0}^{y} \sum_{l=0}^{m} \sum_{k=0}^{z} \sum_{r=0}^{k} \sum_{s=0}^{z-k}\left(\begin{array}{c}
x \\
i
\end{array}\right)\left(\begin{array}{c}
y \\
j
\end{array}\right)\left(\begin{array}{c}
m \\
l
\end{array}\right)\left(\begin{array}{c}
z \\
k
\end{array}\right)\left(\begin{array}{c}
k \\
r
\end{array}\right)\left(\begin{array}{c}
z-k \\
s
\end{array}\right)(-1)^{x+y+z-i-j-r-s} \\
p^{a+x+l+k-1}(1-p)^{b+y+z+m-l-k-1} e^{-\lambda_{r} t\left(\frac{\tau}{t}+x-i+k-r+l\right)} e^{-\lambda_{b} t\left(\frac{(\theta-\tau)}{t}+y-j+z-k-s+m-l\right)} \\
\mathbb{1}_{(0 \leq \alpha \leq 1)} \mathbb{1}_{(0 \leq p \leq 1)} \mathbb{1}_{\left(\lambda_{r}>\lambda_{b}>0\right)}
\end{array}
$$

O detalhamento deste desenvolvimento encontra-se no apêndice A.

Nota-se que a manipulação da expressão 3.7 torna-se mais complicada quanto maior o valor de $N$. Isso é devido, especialmente, ao número de parcelas envolvidas no conjunto de somatórias da expressão 3.7, dado por $(x+1)(y+1)(m+1)\left[2^{z}+z(z+1)\right]$. É possível ver, na tabela 3.1, alguns exemplos da quantidade de parcelas envolvidas dados valores de $x, y, z$ e $m$.

Em 3.1, foi apresentado o cálculo do custo por peça de um processo de estresse. Caso não tenha sido realizado nenhum processo de estresse, pode-se determinar o valor de $t$ que minimiza a expressão 3.1 por meio das distribuições a priori de $p, \lambda_{b}$ e $\lambda_{r}$. Tal cálculo pode ser feito via simulação, gerando valores de $p, \lambda_{b}$ e $\lambda_{r}$ e calculando $\mathbb{E}\left[C\left(t, p, \lambda_{b}, \lambda_{r}\right)\right]$ por meio de um procedimento de Monte Carlo. Caso se utilize as prioris apresentadas em 3.2, Barlow et al. (1994) apresentam analiticamente a expressão da esperança, como função de $t$ :

$$
\mathbb{E}[C(t)]=c_{3}+c_{2} \frac{b}{a+b}+\left(c_{1}+c_{4}-c_{3}\right) \frac{a}{a+b} \frac{\theta \tau}{(\tau+t)(\theta+t)}+\left(c_{4}-c_{2}-c_{3}\right) \frac{b}{a+b} \frac{\theta}{\theta+\tau}
$$

O tempo ótimo obtido a priori pode ser utilizado para a realização do primeiro procedimento de estresse. Após o procedimento, e observados $x, y, z$ e $m$, é possível utilizar um algoritmo do tipo ABC para simular observações da posteriori de $\left(p, \lambda_{b}, \lambda_{r}\right)$, pois verifica-se que a expressão 3.7 é de difícil tratamento analítico. Assim, pode-se novamente utilizar um procedimento de Monte Carlo para encontrar $t$ que minimiza 3.1, obtendo-se assim o tempo ótimo, a posteriori, de duração para futuros testes de estresse. Esse estudo é detalhado no capítulo seguinte. 


\section{Capítulo 4}

\section{Aplicação de ABC em ESS: caso de 2 tipos de peças}

O uso de algum algoritmo do tipo $\mathrm{ABC}$ para o problema descrito no capítulo 3 é adequado, uma vez que a posteriori obtida em 3.7 é de complicado tratamento analítico. Tal dificuldade decorre não só pela quantidade de parcelas envolvidas na expressão, bem como pela presença dos coeficientes binomiais, uma vez que envolvem o cálculo de fatoriais - inviável para valores muito grandes.

Algum algoritmo do tipo Markov Chain Monte Carlo (MCMC), como Gibbs ou MetropolisHastings, não é adequado para este problema, pois seu uso depende da possibilidade de simularmos as distribuições condicionais dos parâmetros, o que também se mostra inviável dada a expressão 3.7 .

No cenário de ESS, as probabilidades associadas a cada tipo possível de peça descritas na seção 3.2 podem ser vistas como componentes de um vetor de parâmetros, dado por $[(1-\alpha) p(1-$ $\left.\left.e^{-\lambda_{r} t}\right),(1-\alpha)(1-p)\left(1-e^{-\lambda_{b} t}\right), \alpha p\left(1-e^{-\lambda_{r} t}\right)+\alpha(1-p)\left(1-e^{-\lambda_{b} t}\right), p e^{-\lambda_{r} t}+(1-p) e^{-\lambda_{b} t}\right]$, de uma distribuição multinomial. Assim, a verossimilhança em 3.5 corresponde ao núcleo de uma distribuição multinomial com este vetor de parâmetros. Observe que a soma das probabilidades envolvidas é igual a 1.

Primeiramente, usaremos o algoritmo Rejeicão 1, conforme apresentado no capítulo 2, com o intuito de gerarmos uma amostra de tamanho $n$ do vetor de parâmetros $\left(\alpha, p, \lambda_{b}, \lambda_{r}\right)$. Para utilização do algoritmo, deve-se estipular os valores dos parâmetros a priori $a, b, \theta$ e $\tau$. O algoritmo é detalhado a seguir.

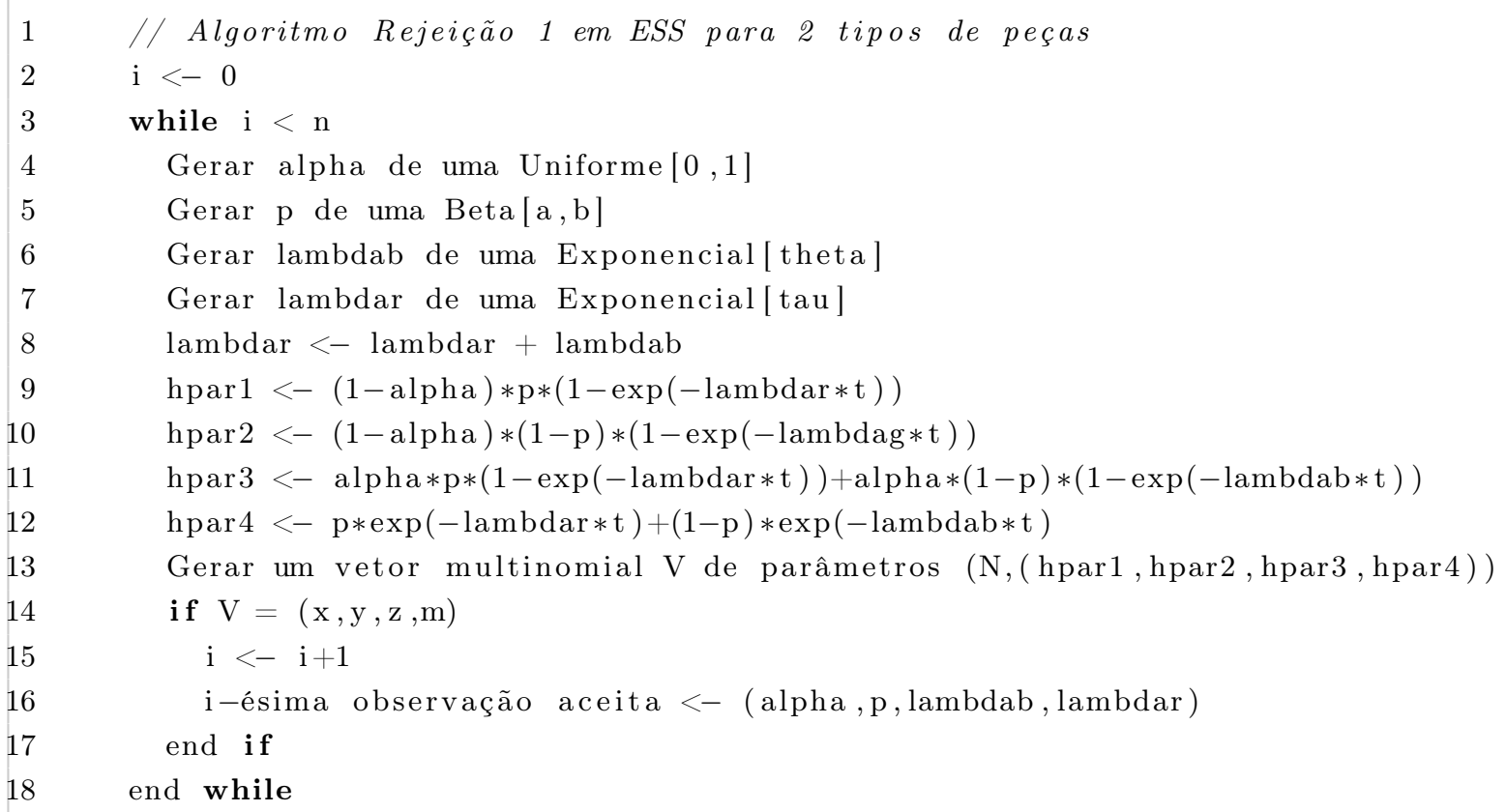


Na sequência, avaliamos a performance do algoritmo Rejeição 1 a partir de um exemplo numérico de teste de estresse com uma quantidade reduzida de peças.

\subsection{Exemplos numéricos para $N$ pequeno}

Vamos considerar uma situação hipotética, na qual uma amostra de 10 peças foi submetida ao estresse por um período de 10 unidades de tempo. Após o término, 4 falharam e seu nível de qualidade era bom, 4 falharam e seu nível de qualidade era ruim, 1 falhou e não foi possível identificar seu nível de qualidade e 1 sobreviveu ao estresse. Assim, o número de parcelas da expressão 3.7 é dado por $(4+1)(4+1)(1+1)\left[2^{1}+1(1+1)\right]=200$. Para a distribuição a priori, utilizamos $a=1$, $b=2, \theta=2$ e $\tau=3$.

A figura 4.1 apresenta os histogramas de 10.000 valores simulados de $\alpha, p, \lambda_{b}$ e $\lambda_{r}$, por meio do algoritmo Rejeição 1. Com estes valores simulados, é possível ainda obter informações a respeito da distribuição a posteriori destes parâmetros, tais como média, mediana, variância, etc.
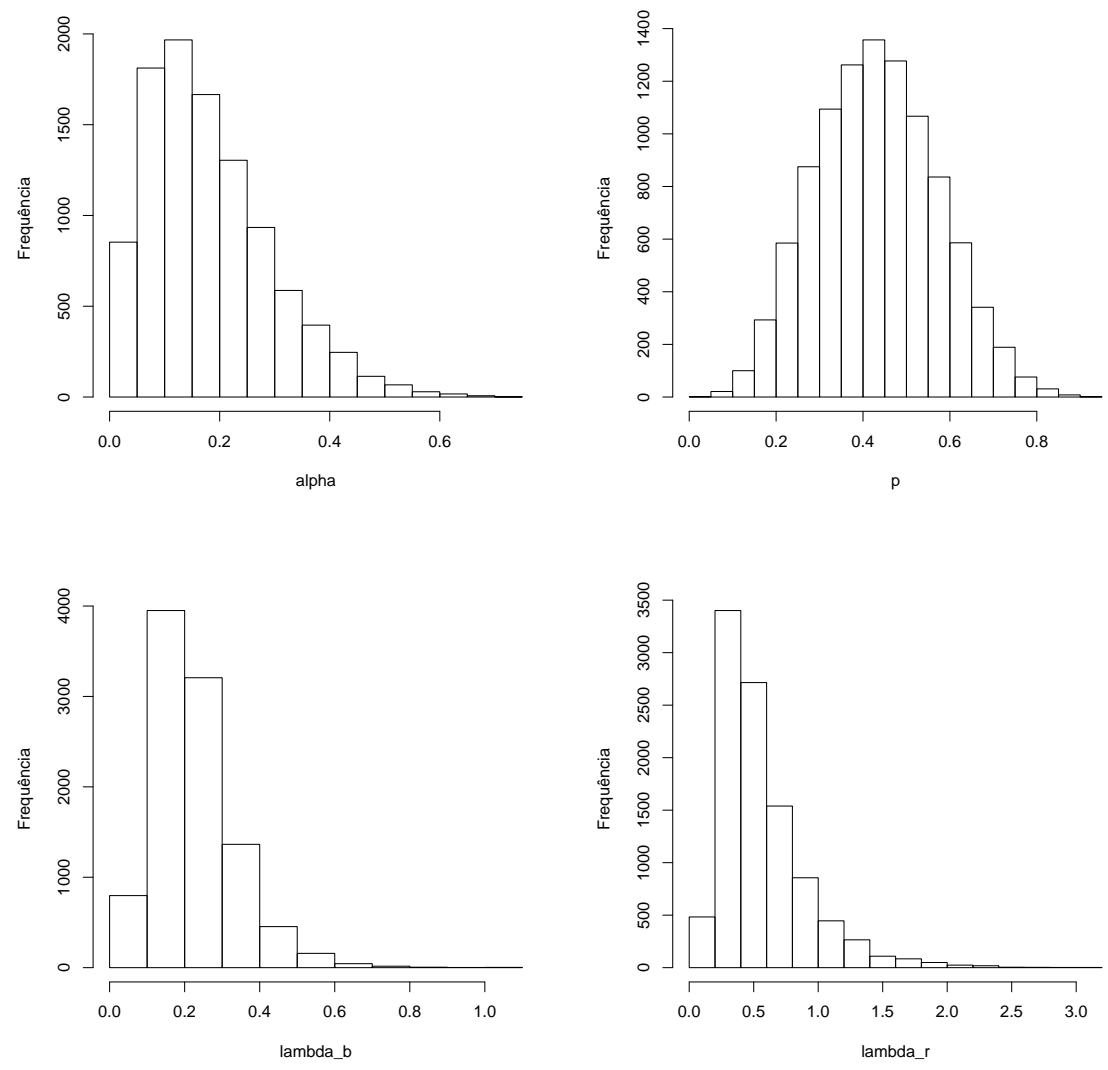

Figura 4.1: Histograma dos valores simulados de $\alpha, p, \lambda_{b}$ e $\lambda_{r}$.

\subsubsection{Comparação com distribuições exatas}

Para verificar a adequação do algoritmo 1, vamos utilizar as distribuições marginais a posteriori exatas dos parâmetros $\alpha, p, \lambda_{b}$ e $\lambda_{r}$. As expressões abaixo foram obtidas a partir da expressão 3.7 e o detalhamento dos cálculos se encontra no apêndice B.

Para $\alpha$, temos que:

$$
f(\alpha \mid x, y, z, m) \propto \alpha^{z}(1-\alpha)^{x+y} \mathbb{1}_{(0 \leq \alpha \leq 1)}
$$

Calculamos o valor da expressão 4.1 para 10.000 pontos distribuídos em $(0,1)$ e normalizamos 
para que a soma dos valores fosse igual a 1, aproximando, assim, a uma densidade. Por fim, acumulamos os valores das densidades para comparar com a distribuição acumulada da amostra dos valores de $\alpha$ obtidos através do algoritmo Rejeição 1, como descrito em 4.1. A comparação é ilustrada na figura 4.2 .

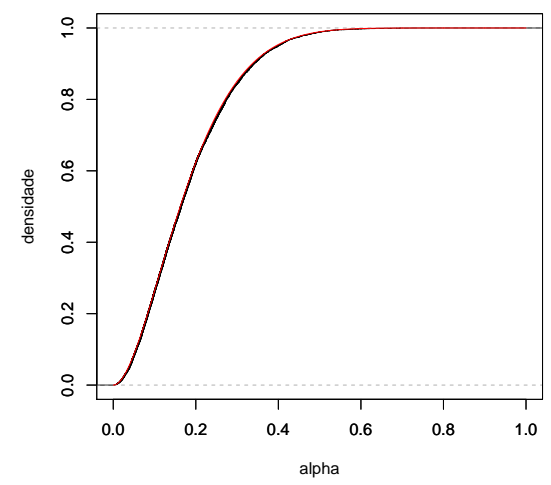

Figura 4.2: Densidade acumulada de $\alpha$ (em vermelho) e densidade simulada (em preto).

Analogamente, procede-se à avaliação para os demais parâmetros. Para a proporção $p$ :

$$
\begin{aligned}
f(p \mid x, y, z, m) \propto \sum_{i=0}^{x} & \sum_{j=0}^{y} \sum_{l=0}^{m} \sum_{k=0}^{z} \sum_{r=0}^{k} \sum_{s=0}^{z-k}\left(\begin{array}{l}
x \\
i
\end{array}\right)\left(\begin{array}{c}
y \\
j
\end{array}\right)\left(\begin{array}{c}
m \\
l
\end{array}\right)\left(\begin{array}{c}
z \\
k
\end{array}\right)\left(\begin{array}{c}
k \\
r
\end{array}\right)\left(\begin{array}{c}
z-k \\
s
\end{array}\right)(-1)^{x+y+z-i-j-r-s} \\
& \frac{p^{a+x+l+k-1}(1-p)^{b+y+z+m-l-k-1}}{[\tau+t(x-i+k-r+l)][\theta+t(x-i+y-j+z-s-r+m)]} \mathbb{1}_{(0 \leq p \leq 1)}
\end{aligned}
$$

Seguindo o feito $\operatorname{com} \alpha$, a figura 4.3 apresenta as densidades acumulada e simulada de $p$.

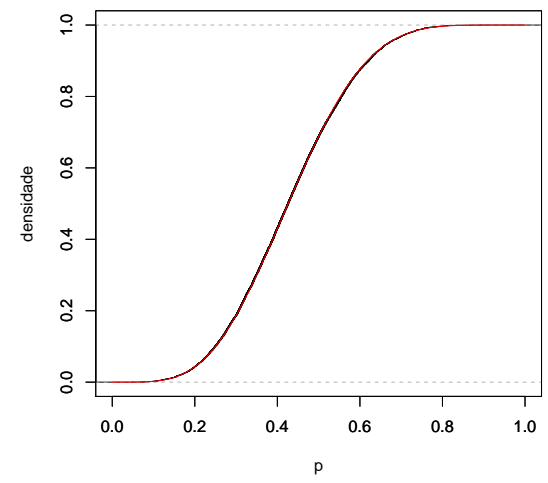

Figura 4.3: Densidade acumulada de $p$ (em vermelho) e densidade simulada (em preto).

Para $\lambda_{b}$ :

$$
\begin{aligned}
f\left(\lambda_{b} \mid x, y, z, m\right) \propto & \sum_{i=0}^{x} \sum_{j=0}^{y} \sum_{l=0}^{m} \sum_{k=0}^{z} \sum_{r=0}^{k} \sum_{s=0}^{z-k}\left(\begin{array}{c}
x \\
i
\end{array}\right)\left(\begin{array}{c}
y \\
j
\end{array}\right)\left(\begin{array}{c}
m \\
l
\end{array}\right)\left(\begin{array}{c}
z \\
k
\end{array}\right)\left(\begin{array}{c}
k \\
r
\end{array}\right)\left(\begin{array}{c}
z-k \\
s
\end{array}\right)(-1)^{x+y+z-i-j-r-s} \\
& \frac{\Gamma(a+x+k+l) \Gamma(b+y+z+m-k-l)}{\Gamma(a+x+b+y+z+m)} \frac{e^{-\lambda_{b} t\left(\frac{\theta}{t}+x+y+z+m-i-j-r-s\right)}}{\tau+t(x-i+k-r+l)} \mathbb{1}_{\left(0<\lambda_{b}\right)}
\end{aligned}
$$




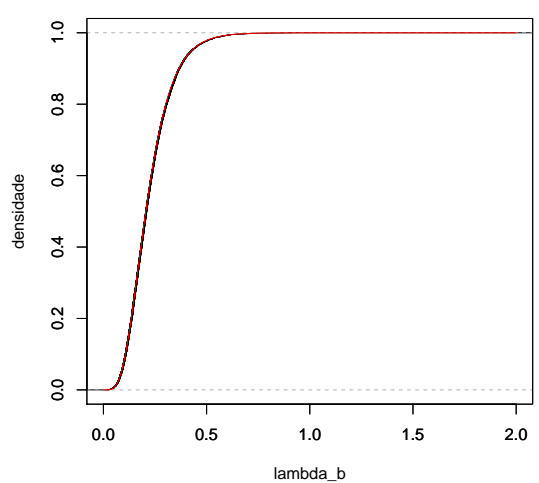

Figura 4.4: Densidade acumulada de $\lambda_{b}$ (em vermelho) e densidade simulada (em preto).

Finalmente, para $\lambda_{r}$ :

$$
\begin{gathered}
f\left(\lambda_{r} \mid x, y, z, m\right) \propto \sum_{i=0}^{x} \sum_{j=0}^{y} \sum_{l=0}^{m} \sum_{k=0}^{z} \sum_{r=0}^{k} \sum_{s=0}^{z-k}\left(\begin{array}{c}
x \\
i
\end{array}\right)\left(\begin{array}{c}
y \\
j
\end{array}\right)\left(\begin{array}{c}
m \\
l
\end{array}\right)\left(\begin{array}{c}
z \\
k
\end{array}\right)\left(\begin{array}{c}
k \\
r
\end{array}\right)\left(\begin{array}{c}
z-k \\
s
\end{array}\right)(-1)^{x+y+z-i-j-r-s} \\
\frac{\Gamma(a+x+k+l) \Gamma(b+y+z+m-k-l)}{\Gamma(a+x+b+y+z+m)} \frac{e^{-\lambda_{r} t\left(\frac{\tau}{t}+x-i+k-r+l\right)}\left[1-e^{-\lambda_{r} t\left(\frac{\theta-\tau}{t}+y-j+z-k-s+m-l\right)}\right]}{\theta-\tau+t(y-j+z-k-s+m-l)} \mathbb{1}_{\left(0<\lambda_{r}\right)}
\end{gathered}
$$

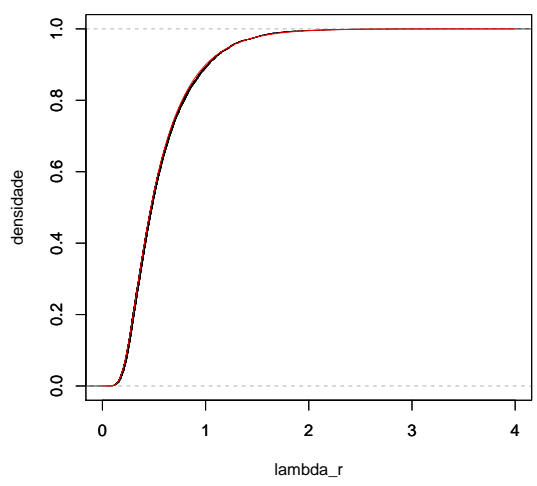

Figura 4.5: Densidade acumulada de $\lambda_{r}$ (em vermelho) e densidade simulada (em preto).

Para os 4 parâmetros, pode-se verificar que a simulação realizada gerou uma amostra bastante fiel à distribuição exata do parâmetro.

\subsubsection{Algoritmo Rejeição 2}

No caso do algoritmo Rejeição 1, aceitamos apenas os candidatos para os quais o vetor simulado foi exatamente igual ao vetor observado no teste de estresse, igual a $(4,4,1,1)$. Iremos agora utilizar o algoritmo Rejeição 2 para avaliar, visualmente, a influência do limite de tolerância $\varepsilon$ no resultado do algoritmo. A função de comparação será a distância euclidiana. 


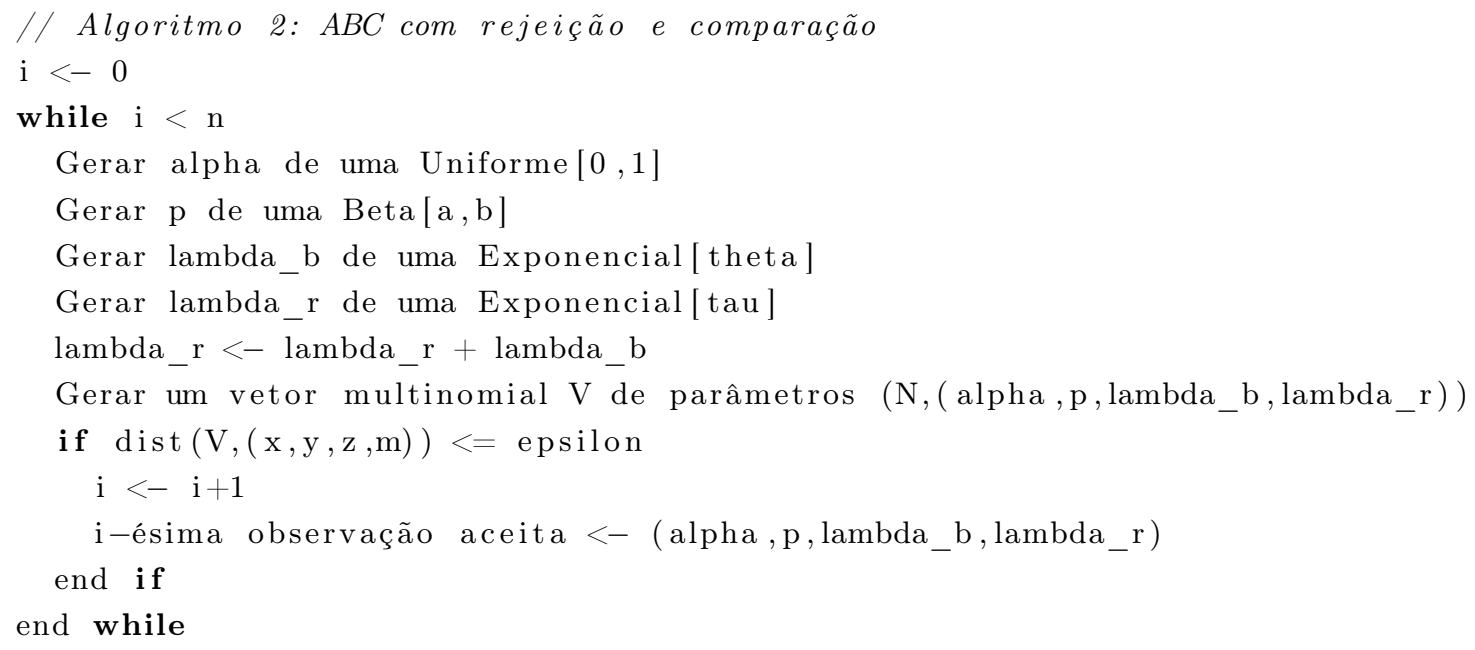

O número de elementos do conjunto $\mathbb{T}_{N}^{4}=\left\{\left(a_{1}, a_{2}, a_{3}, a_{4}\right) \in \mathbb{N}^{4}: a_{1}+a_{2}+a_{3}+a_{4}=N\right\}$ é dado por $\left(\begin{array}{c}N+3 \\ 3\end{array}\right)=(N+3)(N+2)(N+1) / 6, \forall N \in \mathbb{N}$. Desta forma, para $N=10$, existem 286 possíveis resultados distintos para o resultado do vetor $(x, y, z, m)$ simulado. A figura 4.6 apresenta o histograma da distância euclidiana entre os elementos de $\mathbb{T}_{10}^{4}$ e o vetor observado $(4,4,1,1)$.

Temos que, para $\varepsilon=1$, apenas 1 candidato ( $0,3 \%$ do total de 286 possibilidades) seria aceito pelo algoritmo, justamente o vetor observado $(4,4,1,1)$ (caso equivalente ao algoritmo Rejeição 1). Para $\varepsilon=3$, já seriam aceitos $15 \%$ dos candidatos, enquanto que para $\varepsilon=6$ a aceitação é de $71 \%$. Desta forma, fica clara a relação entre a escolha de $\varepsilon$ e a eficiência, em termos de tempo, do algoritmo.

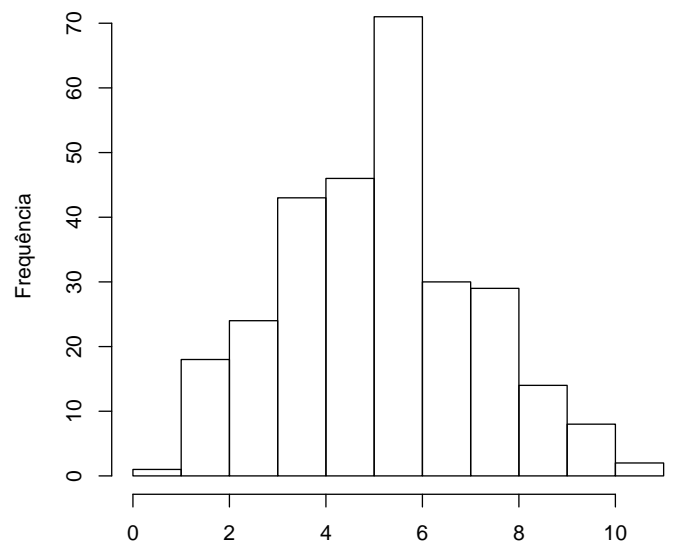

Figura 4.6: Histograma da distância euclidiana entre os elementos de $\mathbb{T}_{10}^{4}$ e $(4,4,1,1)$

A figura 4.7 apresenta as densidades acumuladas simuladas dos parâmetros, variando-se o valor de $\varepsilon$, para $n=10000$.

As linhas apresentadas em laranja representam a densidade acumulada a priori dos parâmetros. Verifica-se que, para $\alpha$ e $p$, quanto menor o valor de $\varepsilon$, maior a proximidade da densidade simulada com a densidade a posteriori exata (em vermelho). Para os parâmetros $\lambda_{b}$ e $\lambda_{r}$, verifica-se que apenas quando $\varepsilon=1$ (caso em que apenas o vetor simulado é igual ao observado, conforme figura 4.6), 

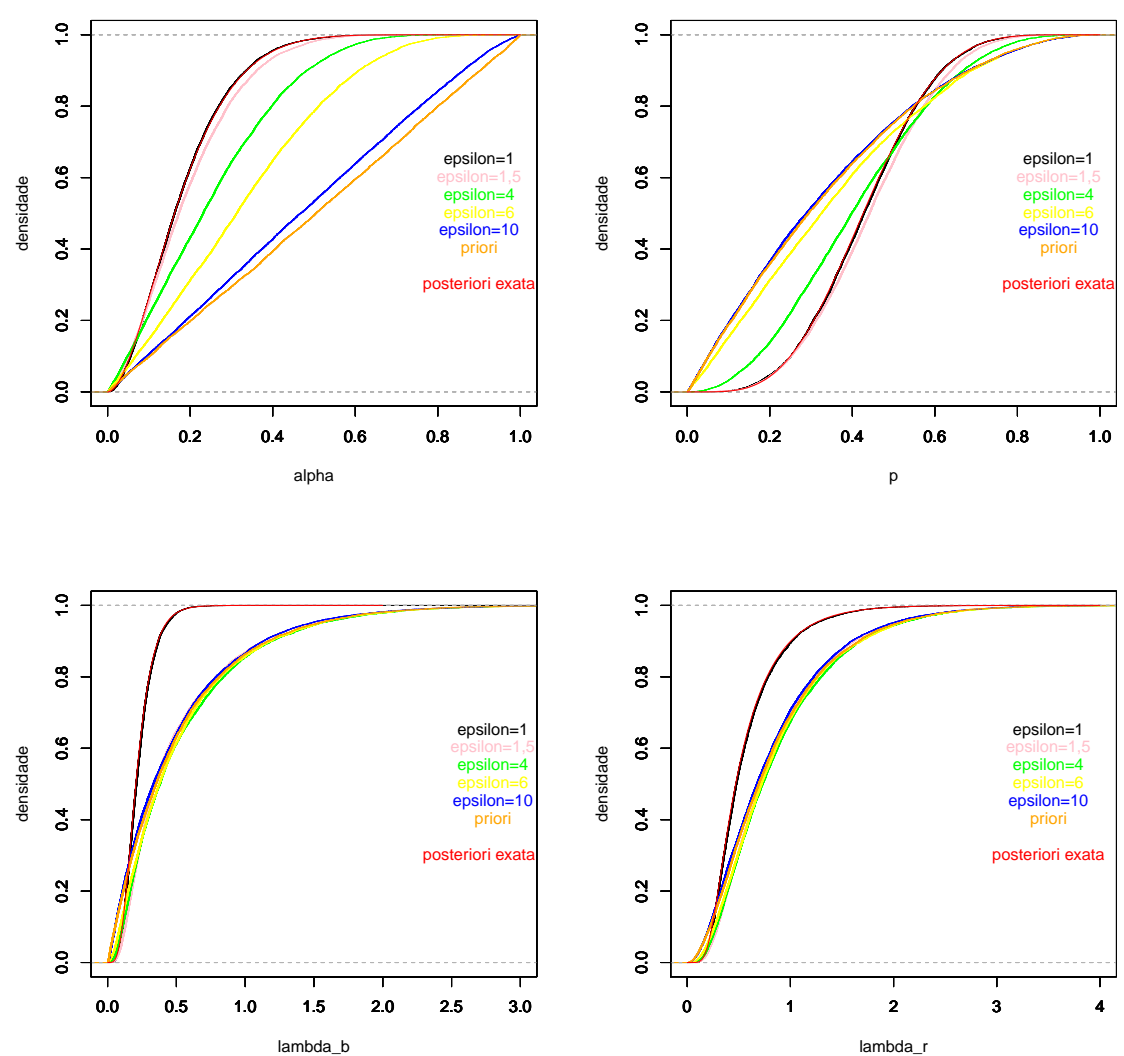

Figura 4.7: Densidades simuladas de $\alpha, p, \lambda_{b}$ e $\lambda_{r}$ para diferentes valores de $\varepsilon$

a distribuição fica similar à posteriori. Como os dados considerados no exemplo de ESS são discretos, ao menos no caso em que $N$ é pequeno o algoritmo demonstra pouca sensibilidade ao valor de $\varepsilon$.

\subsection{Exemplos numéricos para $N$ grande}

Para $N$ grande, o algoritmo Rejeição 1 torna-se ineficiente, pois a chance de sorteamos exatamente o vetor observado reduz-se consideravelmente. Assim, faz-se necessário o uso do algoritmo Rejeição 2, menos restrito, aceitando vetores simulados próximos ao observado.

Para nosso exemplo, utilizaremos como função de comparação a distância euclidiana, e estabelecemos $\varepsilon=0,01 * N$. Vamos considerar a seguinte situação: um lote de 1000 peças será submetido ao estresse. Para a distribuição a priori, utilizamos $a=1, b=2, \theta=2$ e $\tau=3$. Para os custos, consideraremos os valores apresentados em Barlow et al. (1994), onde $c_{1}=100, c_{2}=20, c_{3}=1 \mathrm{e}$ $c_{4}=0,01$.

Considerando-se tais prioris, vamos calcular, por meio de uma simulação de Monte Carlo, o valor da expressão 3.8 para qualquer valor de $t$. Com isso, pode-se encontrar o valor ótimo de $t$ a priori que minimiza o valor esperado da função de custo. A figura 4.8 apresenta os valores esperados (a priori) da função de custo calculados para $t$ variando de 0 a 30 unidades de tempo.

$\mathrm{O}$ valor de $t$ que minimiza o valor esperado da função de custo, a priori, é de 10,6 unidades de tempo. Pode-se provar que este valor é mínimo para qualquer $t>0$. Desta forma, o procedimento de estresse será realizado durante 10,6 unidades de tempo. Após o término do procedimento de estresse, observou-se que 167 peças não sobreviveram ao estresse e seu nível de qualidade foi verificado como 


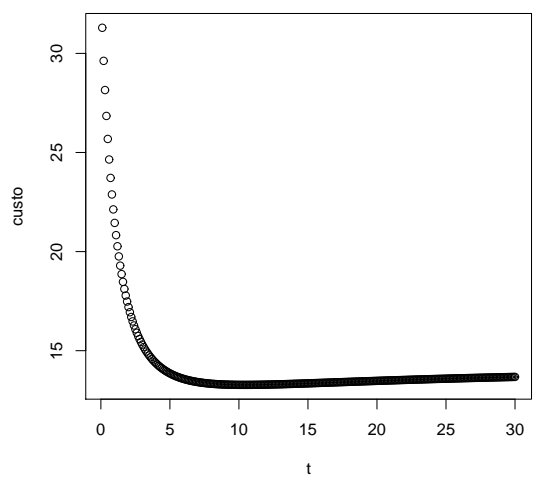

Figura 4.8: Função de risco a priori

ruim, 332 peças não sobreviveram e verificou-se seu nível de qualidade como bom. Outras 498 peças não sobreviveram ao estresse, porém não foi possível verificar seu nível de qualidade e, por fim, observou-se que 3 peças sobreviveram ao estresse. Em resumo, $x=167, y=332, z=498$ e $m=3$. Neste caso, a quantidade de parcelas a serem somadas de acordo com a expressão 3.7 da posteriori conjunta dos parâmetros seria incalculável, devido ao fator $2^{z}$ presente na expressão.

Desta forma, para gerar observações desta posteriori, iremos utilizar o ABC. A figura 4.9 apresenta os histogramas de 10.000 valores simulados de $\alpha, p, \lambda_{b}$ e $\lambda_{r}$ por meio do Algoritmo Rejeição 2. Com estes valores simulados, é possível obter informações a respeito da distribuição a posteriori destes parâmetros, tais como média, mediana, variância, etc.

\subsubsection{Influência de $\varepsilon$}

Para verificar a influência de $\varepsilon$ no resultado final da posteriori, aplicamos o algoritmo Rejeição 2 conforme a seção 4.2 , porém para valores distintos de $\varepsilon$ variando de 10 até 1000 . Na figura 4.10 apresentamos as densidades acumuladas dos parâmetros, a posteriori, simuladas a partir de cada valor de $\varepsilon$, bem como as densidades acumuladas a partir da distribuição a priori.

Pode-se verificar que, para os 4 parâmetros, quanto menor o valor de $\varepsilon$, mais a densidade acumulada simulada "se afasta" da densidade acumulada a priori, intuitivamente aproximando-se da densidade a posteriori exata.

\subsubsection{Função de custo}

Na figura 4.11, apresentamos graficamente os valores esperados da função de custo por peça (conforme expressão 3.1), calculados via método de Monte Carlo, para valores de $t$ variando de 0 a 30 unidades de tempo. Tal cálculo é efetuado a partir da amostra obtida pelo algoritmo descrito na seção 4.2. Conclui-se então que o $t$ ótimo de estresse (a posteriori), correspondente ao mínimo dos valores esperados observados na figura, é de 5,4 unidades de tempo. Este valor é o mínimo da função de custo para qualquer $t>0$.

Portanto, para o exemplo considerado, a utilização do algoritmo $\mathrm{ABC}$ viabiliza a simulação da posteriori dos parâmetros $p, \lambda_{r}$ e $\lambda_{b}$, a partir da informação obtida com o teste de estresse das 1.000 peças. Por fim, a revisão da incerteza sobre tais parâmetros leva a uma redução de $51 \%$ no 

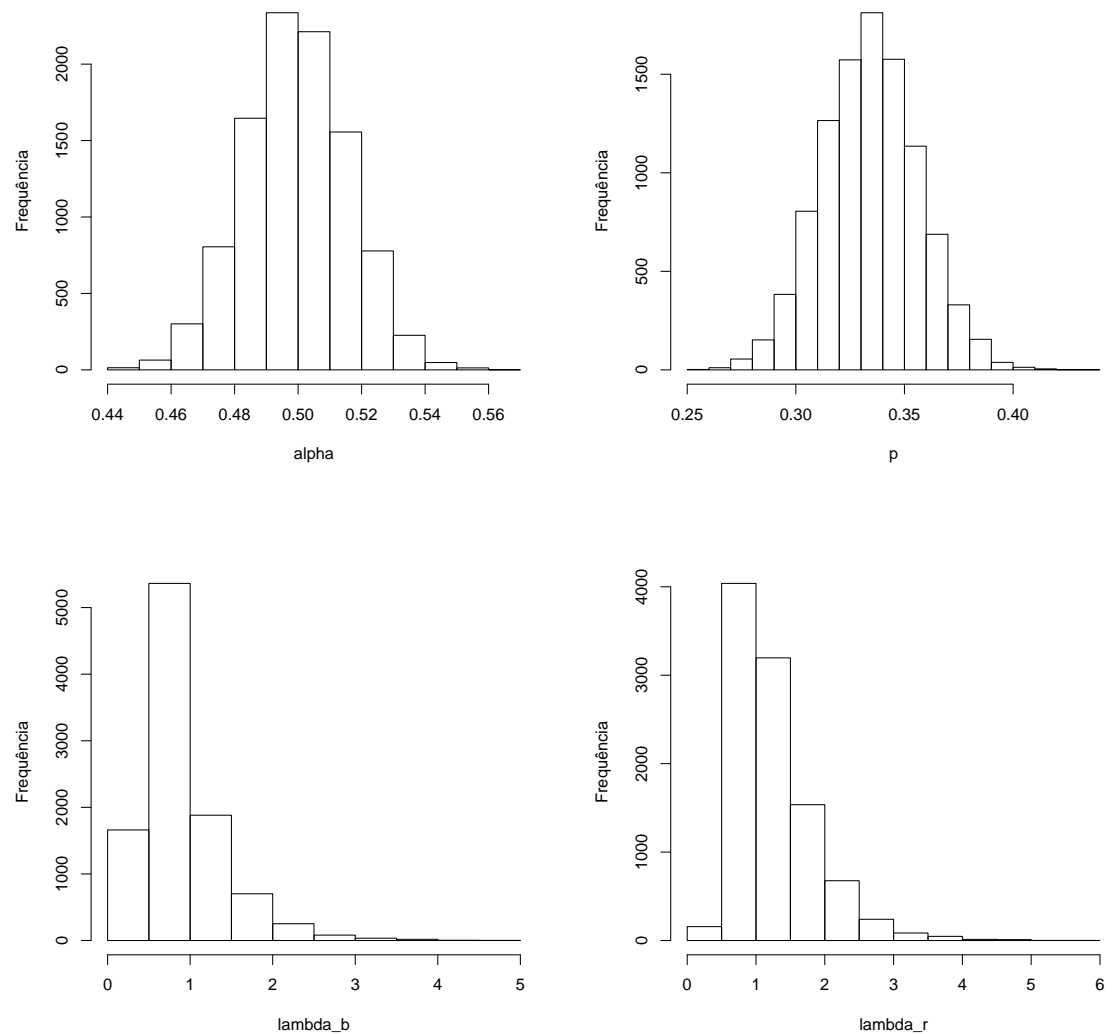

Figura 4.9: Histograma dos valores simulados de $\alpha, p, \lambda_{b} e \lambda_{r}$.

tempo necessário para a duração do estresse, de 10,6 unidades de tempo a priori para 5,4 unidades de tempo a posteriori. 

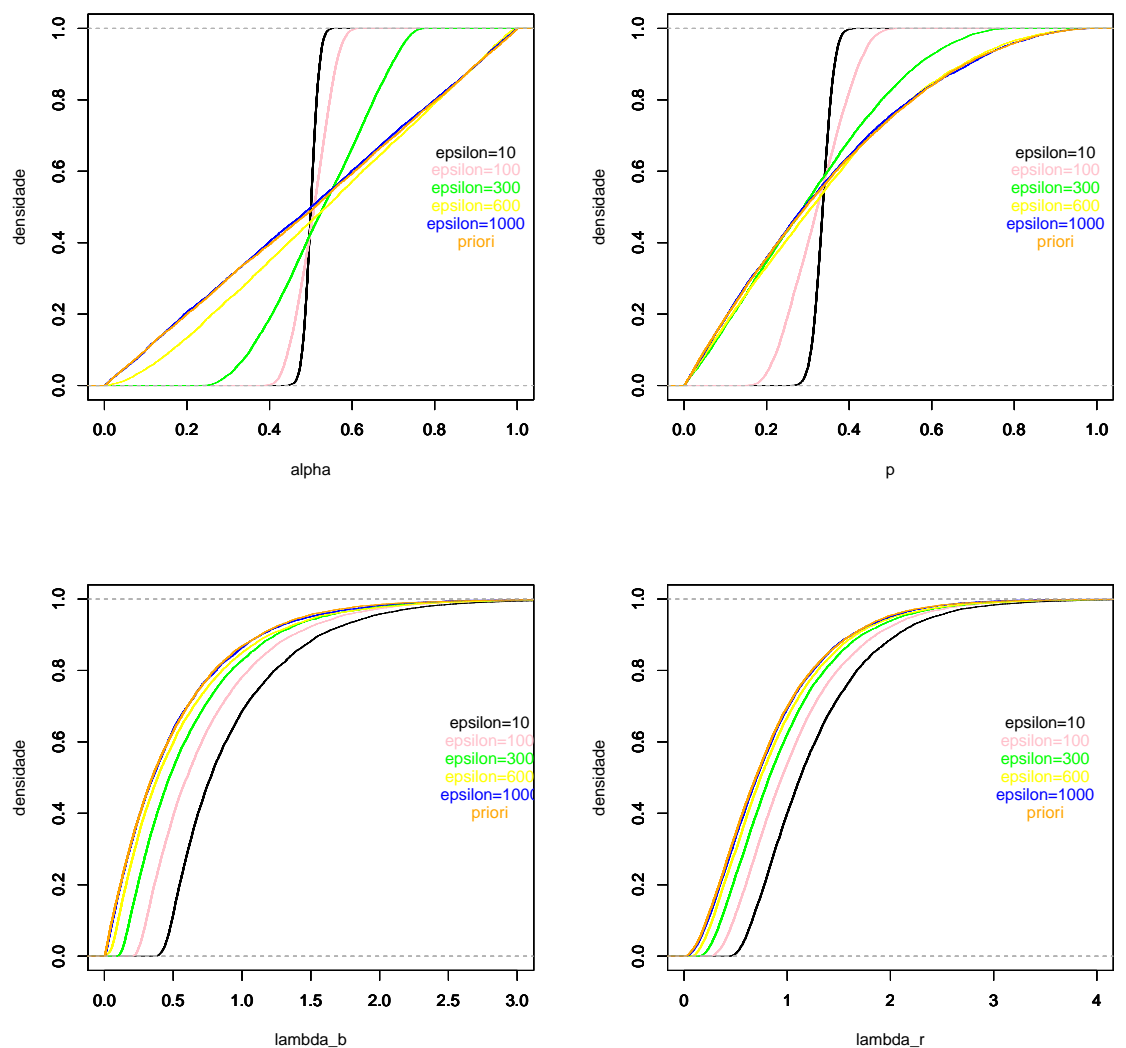

Figura 4.10: Densidades acumuladas a posteriori de $\alpha, p, \lambda_{b}$ e $\lambda_{r}$ simuladas a partir do algoritmo Rejeição 2 para diferentes valores de $\varepsilon$

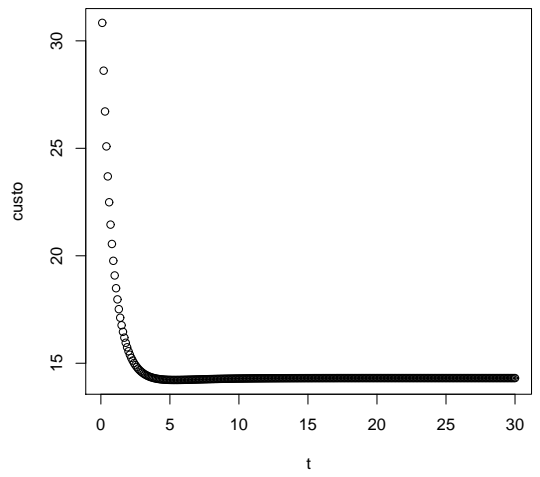

Figura 4.11: Função de risco a posteriori 


\section{Capítulo 5}

\section{Aplicação para $k$ tipos de peças}

No capítulo anterior, estudamos o processo de ESS num cenário com 2 tipos de peças, boas ou ruins. Neste capítulo, propomos um modelo mais geral, no qual consideraremos que cada peça pode ser classificada em um dentre $k$ tipos possíveis. A cada tipo possível de peça está associado um mesmo nível de qualidade, ou seja, duas peças do mesmo tipo possuem a mesma taxa de falha.

Assim, em um lote qualquer de peças, vamos supor que a proporção de peças do tipo 1 é dada por $p_{1}$, a proporção de peças do tipo 2 é dada por $p_{2}$, e assim por diante, com $p_{k}=1-\sum_{i=1}^{k-1} p_{i}$ representando a proporção de peças do $k$-ésimo tipo.

Com relação às taxas de falha, vamos considerar que $\lambda_{1}$ representa a taxa de falha das peças do tipo $1, \lambda_{2}$ representa a taxa de falha das peças do tipo 2 , e assim por diante, até $\lambda_{k}$, que representa a taxa de falha das peças do tipo $k$. Consideraremos também uma relação de ordem nas taxas de falha, ou seja, as peças de tipo 1 representam as peças de melhor qualidade (menor taxa de falha), as peças de tipo 2 representam as peças de segunda melhor qualidade (segunda menor taxa de falha), até as peças de tipo $k$, que representam as peças de pior qualidade (maior taxa de falha). Com isso, temos $\lambda_{1}<\lambda_{2}<\ldots<\lambda_{k}$.

\subsection{Modelagem bayesiana}

\subsubsection{Distribuição a priori dos parâmetros}

Assumiremos que o vetor de parâmetros $\left(p_{1}, \ldots, p_{k-1}\right)$ segue, a priori, uma distribuição Dirichlet de parâmetros $\left(\alpha_{1}, \ldots, \alpha_{k}\right)$, onde $\alpha_{i}>0, \forall i \in\{1, \ldots, k\}$. Ou seja:

$f\left(p_{1}, \ldots, p_{k-1}\right)=\frac{\Gamma\left(\alpha_{1}+\ldots+\alpha_{k}\right)}{\Gamma\left(\alpha_{1}\right) \ldots \Gamma\left(\alpha_{k}\right)} p_{1}^{\alpha_{1}-1} \ldots p_{k-1}^{\alpha_{k-1}-1}\left(1-\sum_{i=1}^{k-1} p_{i}\right)^{\alpha_{k}-1} \mathbb{1}_{\left(0 \leq p_{i} \leq 1, \forall i \in\{1, \ldots, k-1\}\right)} \mathbb{1}_{\left(\sum_{i=1}^{k-1} p_{i} \leq 1\right)}$

Para as taxas de falha, consideramos que, a priori, seguem distribuições condicionais exponenciais deslocadas. Temos que $\lambda_{1}$ é exponencial de parâmetro $\theta_{1}, \theta_{1}>0$, e, para $1<i \leq k$, temos que $\lambda_{i}$, dado $\lambda_{1}, \ldots, \lambda_{i-1}$, é exponencial de parâmetro $\theta_{i}$, deslocada por $\lambda_{i-1}$. Estes deslocamentos resultam em $\lambda_{1}<\ldots<\lambda_{k}$, garantindo a ordenação das taxas de falha. Assim como no caso de 2 tipos de peça, tal especificação significa que, dado o valor mínimo de uma taxa de falha, o processo de estressar as peças não altera tal taxa de falha por conta da propriedade de "falta de memória" da distribuição exponencial. 
Assim:

$$
f\left(\lambda_{1}, \ldots, \lambda_{k}\right)=f\left(\lambda_{1}\right) f\left(\lambda_{2} \mid \lambda_{1}\right) \ldots f\left(\lambda_{k} \mid \lambda_{k-1}\right)=\theta_{1} e^{-\theta_{1} \lambda_{1}} \theta_{2} e^{-\theta_{2}\left(\lambda_{2}-\lambda_{1}\right)} \ldots \theta_{k} e^{-\theta_{k}\left(\lambda_{k}-\lambda_{k-1}\right)} \mathbb{1}_{\left(0<\lambda_{1}<\ldots<\lambda_{k}\right)}
$$

Assumindo independência entre $\left(p_{1}, \ldots, p_{k-1}\right)$ e $\left(\lambda_{1}, \ldots, \lambda_{k}\right)$, temos que a priori conjunta é dada por:

$$
\begin{array}{r}
f\left(p_{1}, \ldots, p_{k-1}, \lambda_{1}, \ldots, \lambda_{k}\right) \propto p_{1}^{\alpha_{1}-1} \ldots p_{k-1}^{\alpha_{k-1}-1}\left(1-\sum_{i=1}^{k-1} p_{i}\right)^{\alpha_{k}-1} e^{-\theta_{1} \lambda_{1}} e^{-\theta_{2}\left(\lambda_{2}-\lambda_{1}\right)} \ldots e^{-\theta_{k}\left(\lambda_{k}-\lambda_{k-1}\right)} \\
\mathbb{1}_{\left(0 \leq p_{i} \leq 1, \forall i \in\{1, \ldots, k-1\}\right)} \mathbb{1}_{\left(\sum_{i=1}^{k-1} p_{i} \leq 1\right)} \mathbb{1}_{\left(0<\lambda_{1}<\ldots<\lambda_{k}\right)}
\end{array}
$$

\subsubsection{Função de verossimilhança}

Vamos considerar o experimento em que um lote de $N$ peças será submetido a um estresse de duração de $T$ unidades de tempo. Após o término do estresse, podemos observar, para cada peça, se esta falhou ou não e, em caso de falha, podemos submeter a peça a uma autópsia para verificar qual o seu nível de qualidade. Assim como no capítulo 4, vamos considerar que o processo de autópsia pode não permitir, com probabilidade $\alpha$, identificar a qualidade de uma peça que falhou.

Consideraremos, para as peças submetidas ao processo de estresse, os vetores aleatórios $Y_{1}, Y_{2}, \ldots, Y_{N}$, em que $Y_{j}=\left(Y_{j 1}, Y_{j 2}, Y_{j 3}\right), \forall j \in\{1, \ldots, N\}$, onde

- $Y_{j 1}=\left\{\begin{array}{l}1, \text { se a } j \text {-ésima peça falhou durante o processo de estresse } \\ 0, \text { caso contrário. }\end{array}\right.$

- $Y_{j 2}=\left\{\begin{array}{l}1, \text { se foi possível verificar a qualidade da } j \text {-ésima peça } \\ 0, \text { caso contrário. }\end{array}\right.$

- $Y_{j 3}=$ tipo da $j$-ésima peça, com $Y_{j 3} \in\{1, \ldots, k\}$.

Suponha que $Y_{1}, \ldots, Y_{N}$, dado $\theta=\left(\alpha, p_{1}, \ldots, p_{k-1}, \lambda_{1}, \ldots, \lambda_{k}\right)$, são condicionalmente independentes e identicamente distribuídos. Desta forma, temos as seguintes probabilidades, $\forall j \in\{1, \ldots, N\}$ :

- $\mathbb{P}\left(Y_{j}=(1,1, i) \mid \theta\right)=p_{i}\left(1-e^{-\lambda_{i} t}\right)(1-\alpha), \forall i \in\{1, \ldots, k\}$

- $\mathbb{P}\left(Y_{j}=(1,0, i) \mid \theta\right)=p_{i}\left(1-e^{-\lambda_{i} t}\right) \alpha, \forall i \in\{1, \ldots, k\}$

- $\mathbb{P}\left(Y_{j}=(0,0, i) \mid \theta\right)=p_{i} e^{-\lambda_{i} t}, \forall i \in\{1, \ldots, k\}$

Para as probabilidades acima, consideramos que $p_{k}=1-\sum_{i=1}^{k-1} p_{i}$. Vale ressaltar que, em alguns casos, a terceira componente do vetor não é registrada. Isto ocorre caso a peça sobreviva ao estresse, 
ou caso a peça falhe no estresse porém não seja possível identificar seu tipo pela autópsia.

Assim, a partir do experimento, pode-se registrar $D=\left(x_{1}, \ldots, x_{k}, z, m\right)$, onde:

- $x_{1}=\sum_{j=1}^{N} \mathbb{1}_{\left(Y_{j}=(1,1,1)\right)}$ : número de peças que falharam no estresse e foram verificadas como do tipo 1 ;

- $x_{k}=\sum_{j=1}^{N} \mathbb{1}_{\left(Y_{j}=(1,1, k)\right)}$ : número de peças que falharam no estresse e foram verificadas como do tipo $k$;

- $z=\sum_{j=1}^{N} \mathbb{1}_{\left(\left(Y_{j 1}, Y_{j 2}\right)=(1,0)\right)}$ : número de peças que falharam no estresse porém não foram identificadas na autópsia;

- $m=\sum_{j=1}^{N} \mathbb{1}_{\left(\left(Y_{j 1}, Y_{j 2}\right)=(0,0)\right)}$ : número de peças que sobreviveram ao estresse.

Portanto $D \in\left\{\left(a_{1}, \ldots, a_{k}, a_{k+1}, a_{k+2}\right) \in \mathbb{N}^{k+2}: a_{1}+\ldots+a_{k}+a_{k+1}+a_{k+2}=N\right\}$.

Temos que a verossimilhança dos dados é dada por:

$$
\begin{aligned}
& L\left(\theta \mid\left(x_{1}, \ldots, x_{k}, z, m\right)\right)=\mathbb{P}\left(D=\left(x_{1}, \ldots, x_{k}, z, m\right) \mid p_{1}, \ldots, p_{k-1}, \lambda_{1}, \ldots, \lambda_{k}\right) \propto \\
& \propto \prod_{i=1}^{k}\left[p_{i}\left(1-e^{-\lambda_{i} t}\right)(1-\alpha)\right]^{x_{i}}\left[\sum_{i=1}^{k} p_{i}\left(1-e^{-\lambda_{i} t}\right) \alpha\right]^{z}\left[\sum_{i=1}^{k} p_{i} e^{-\lambda_{i} t}\right]^{m}
\end{aligned}
$$

Assumimos, a priori, que $\alpha$ possui distribuição uniforme em $[0,1]$.

Enfim, chegamos à expressão da função densidade a posteriori dos parâmetros, dada por:

$$
\begin{aligned}
& f\left(\alpha, p_{1}, \ldots, p_{k-1}, \lambda_{1}, \ldots, \lambda_{k} \mid x_{1}, \ldots, x_{k}, z, m\right) \propto \\
& \propto \alpha^{z}(1-\alpha)^{\sum_{i=1}^{k} x_{i}} \sum_{i_{1}=0}^{x_{1}} \ldots \sum_{i_{k}=0}^{x_{k}} \sum_{|\beta|=z} \sum_{j_{1}=0}^{\beta_{1}} \ldots \sum_{j_{k}=0}^{\beta_{k}} \sum_{|\gamma|=m}\left(\begin{array}{c}
x_{1} \\
i_{1}
\end{array}\right) \ldots\left(\begin{array}{c}
x_{k} \\
i_{k}
\end{array}\right)\left(\begin{array}{c}
z \\
|\beta|
\end{array}\right)\left(\begin{array}{c}
\beta_{1} \\
j_{1}
\end{array}\right) \ldots\left(\begin{array}{c}
\beta_{k} \\
j_{k}
\end{array}\right)\left(\begin{array}{c}
m \\
|\gamma|
\end{array}\right) \\
& p_{1}^{\left(\alpha_{1}+x_{1}+\beta_{1}+\gamma_{1}-1\right)} \ldots p_{k}^{\left(\alpha_{k}+x_{k}+\beta_{k}+\gamma_{k}-1\right)}(-1)^{\sum_{l=1}^{k}\left(x_{l}+\beta_{l}-i_{l}-j_{l}\right)} \\
& e^{-\lambda_{1} t\left(\frac{\theta_{1}-\theta_{2}}{t}+x_{1}-i_{1}+\beta_{1}-j_{1}+\gamma_{1}\right)} \ldots e^{-\lambda_{k-1} t\left(\frac{\theta_{k-1}-\theta_{k}}{t}+x_{k-1}-i_{k-1}+\beta_{k-1}-j_{k-1}+\gamma_{k-1}\right)} e^{-\lambda_{k} t\left(\frac{\theta_{k}}{t}+x_{k}-i_{k}+\beta_{k}-j_{k}+\gamma_{k}\right)} \\
& \mathbb{1}_{\left(0 \leq p_{i} \leq 1, \forall i \in\{1, \ldots, k\}\right)} \mathbb{1}_{\left(\sum_{i=1}^{k} p_{i}=1\right)} \mathbb{1}_{\left(0<\lambda_{1}<\ldots<\lambda_{k}\right)} \mathbb{1}_{(0<\alpha<1)}
\end{aligned}
$$


O detalhamento desta expressão encontra-se no apêndice C. Nesta expressão, temos que $|\gamma|=m$ representa o conjunto $\left\{\left(\gamma_{1}, \ldots, \gamma_{k}\right) \in \mathbb{N}^{k}: \sum_{i=1}^{k} \gamma_{i}=m\right\}$, bem como $\left(\begin{array}{c}m \\ |\gamma|\end{array}\right)=\frac{m !}{\gamma_{1} ! \ldots \gamma_{k} !}$. Também consideramos $p_{k}=1-\sum_{i=1}^{k-1} p_{i}$

\subsubsection{Função de custo}

Para o caso de $k$ tipos de falha, não estamos classificando as peças binariamente como boas ou ruins, e sim ordenando seu nível de qualidade pelas respectivas taxas de falha. Com esta generalização, propomos uma estrutura de custos da seguinte forma: $c_{i}$ representa o custo de se estressar uma peça do tipo $i$ e esta sobreviver e $d_{i}$ representa o custo de se estressar uma peça do tipo $i$ e esta falhar, com $i$ variando de 1 a $k$.

É intuitivo supor então que $c_{1}<c_{2}<\ldots<c_{k}$, pois quanto pior a qualidade da peça, maior o custo desta sobreviver ao processo. E supomos também que $d_{1}>d_{2}>\ldots>d_{k}$, pois quanto melhor a qualidade da peça, maior o custo desta falhar no estresse. Também vamos considerar que $e_{1}$ é o custo de se estressar qualquer peça e esta sobreviver, e que $e_{2}$ é o custo de se estressar qualquer peça e esta falhar.

Caso apenas as peças do tipo 1 sejam consideradas de qualidade aceitável, e todas as peças de qualquer outro tipo forem consideradas indesejáveis no processo final, poderíamos considerar $c_{1}=0$ e $d_{2}=d_{3}=\ldots=d_{k}=0$. Obteríamos, assim, uma estrutura de custos similar à apresentada no capítulo 3 para o caso de 2 tipos de peças.

A partir dos custos $c_{1}, \ldots, c_{k}, d_{1}, \ldots, d_{k}, e_{1}, e_{2}$, temos que o custo total de um teste de estresse de duração $T=t$, é dado por:

$$
C_{t}\left(Y_{1}, \ldots, Y_{N}\right)=\sum_{i=1}^{k}\left[\left(d_{i}+e_{2}\right) \sum_{j=1}^{N} \mathbb{1}_{\left(\left(Y_{j 1}, Y_{j 3}\right)=(1, i)\right)}\right]+\sum_{i=1}^{k}\left[\left(c_{i}+e_{1}\right) \sum_{j=1}^{N} \mathbb{1}_{\left(\left(Y_{j 1}, Y_{j 3}\right)=(0, i)\right)}\right]
$$

Assim, a esperança condicional, dado $\theta$, do custo de um teste de estresse de duração $T=t$, é dada por:

$$
\begin{array}{r}
E\left[C_{t} \mid \theta\right]= \\
=\sum_{i=1}^{k}\left[\left(d_{i}+e_{2}\right) \sum_{j=1}^{N} \mathbb{P}\left(Y_{j 1}=1, Y_{j 3}=i \mid \theta\right)\right]+\sum_{i=1}^{k}\left[\left(c_{i}+e_{1}\right) \sum_{j=1}^{N} \mathbb{P}\left(Y_{j 1}=0, Y_{j 3}=i \mid \theta\right)\right]= \\
=\sum_{i=1}^{k}\left[\left(d_{i}+e_{2}\right) N p_{i}\left(1-e^{-\lambda_{i} t}\right)\right]+\sum_{i=1}^{k}\left[\left(c_{i}+e_{1}\right) N p_{i} e^{-\lambda_{i} t}\right]= \\
=N \sum_{i=1}^{k} p_{i}\left[\left(d_{i}+e_{2}\right)\left(1-e^{-\lambda_{i} t}\right)+\left(c_{i}+e_{1}\right) e^{-\lambda_{i} t}\right]
\end{array}
$$


Seja $\bar{C}_{t}$ o custo médio por peça. Assim:

$$
E\left[\bar{C}_{t} \mid \theta\right]=E\left[\frac{C_{t}}{N} \mid \theta\right]=\sum_{i=1}^{k} p_{i}\left[\left(d_{i}+e_{2}\right)\left(1-e^{-\lambda_{i} t}\right)+\left(c_{i}+e_{1}\right) e^{-\lambda_{i} t}\right]
$$

Finalmente, temos que

$$
E\left[\bar{C}_{t}\right]=E\left[E\left[\bar{C}_{t} \mid \theta\right]\right]
$$

Conforme feito no capítulo 4 para 2 tipos de peças, a partir das expressões 5.8 e 5.9, podemos obter o valor de $t$ ótimo que minimize o custo médio por peça a partir da distribuição de $\theta$. A partir da priori de $\theta$, pode-se obter um $t$ ótimo (a priori) para a duração de um primeiro teste de estresse e, a partir dos dados observados, pode-se obter a posteriori de $\theta$ e, novamente, o tempo $t$ ótimo (a posteriori) de duração para futuros testes de estresse. Nesse caso, devemos determinar $t$ tal que $E\left[\bar{C}_{t} \mid D\right]$ seja mínimo.

\subsection{Aplicação do ABC: 3 tipos de peças}

Iremos agora apresentar aplicações dos algoritmos ABC para trabalhar com a expressão 5.5, distribuição a posteriori de $\theta$ dada a informação amostral. Os exemplos numéricos considerarão que $k=3$, embora todos os resultados possam, teoricamente, ser aplicados para qualquer valor de $k \geq 2$.

O algoritmo a seguir representa a aplicação do algoritmo Rejeição 1, apresentado em 2.1, para o caso de 3 tipos de peças. Para simular $\left(p_{1}, p_{2}\right)$ a priori, é preciso simular a distribuição Dirichlet, que faremos por meio do seguinte resultado:

Lema 1 Sejam $W_{1}, \ldots, W_{k}$ variáveis aleatórias independentes, com $W_{1} \sim \operatorname{Gama}\left(\alpha_{1}, \beta\right), \ldots, W_{k} \sim$ $\operatorname{Gama}\left(\alpha_{k}, \beta\right)$. Seja $S=\sum_{i=1}^{k} W_{i}$. Então $W=\left(\frac{W_{1}}{S}, \ldots, \frac{W_{k-1}}{S}\right) \sim \operatorname{Dirichlet}\left(\alpha_{1}, \ldots, \alpha_{k}\right)$.

A prova do lema 1 pode ser encontrada em Devroye (1986).

Para simular $n$ observações a partir do algoritmo Rejeição 1, é necessário estabelecer a priori os valores de $t, \alpha_{1}, \alpha_{2}, \alpha_{3}, \theta_{1}, \theta_{2}$ e $\theta_{3}$.

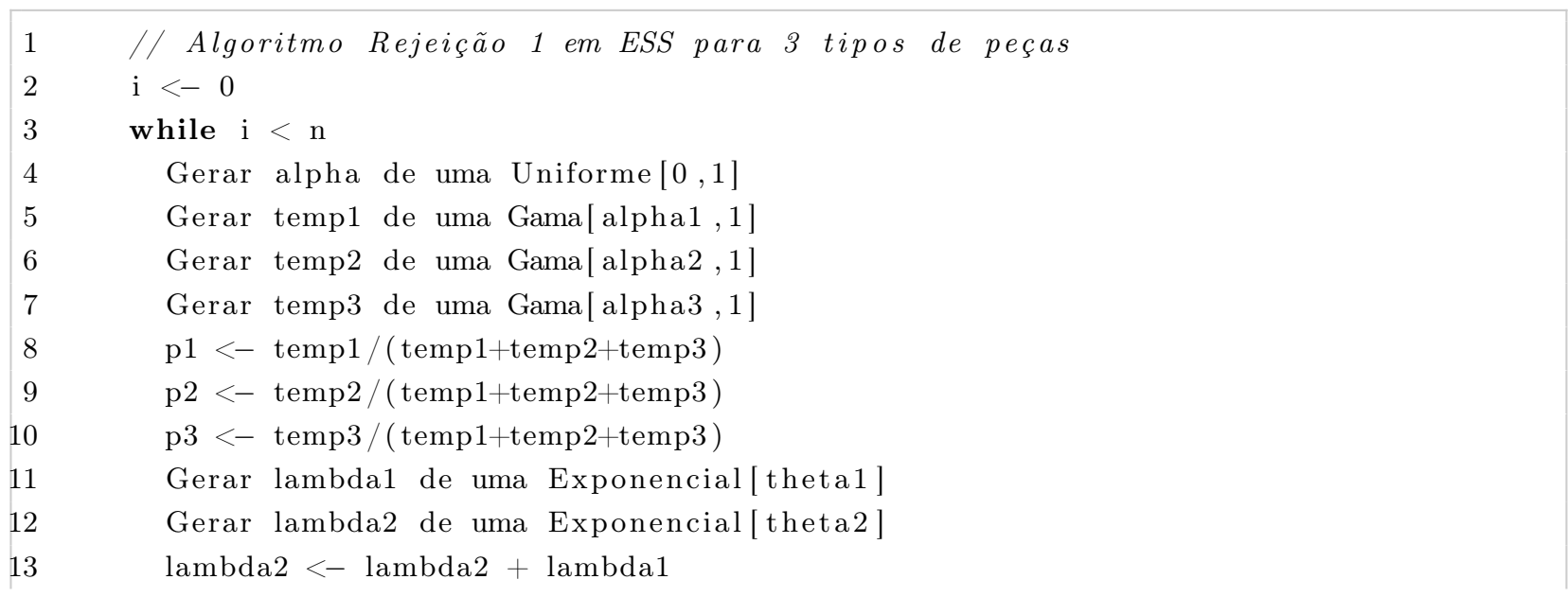


14 Gerar lambda3 de uma Exponencial[theta3]

$15 \quad$ lambda $3<-$ lambda $3+$ lambda 2

$16 \quad$ hpar $1<-(1-$ alpha $) * \mathrm{p} 1 *(1-\exp (-\operatorname{lambda} 1 * \mathrm{t}))$

$17 \quad \operatorname{hpar} 2<-(1-$ alpha $) * \mathrm{p} 2 *(1-\exp (-\operatorname{lambda} 2 * \mathrm{t}))$

$18 \quad \operatorname{hpar} 3<-(1-$ alpha $) * \mathrm{p} 3 *(1-\exp (-\operatorname{lambda} 3 * \mathrm{t}))$

$19 \operatorname{hpar} 4<-$ alpha $* \mathrm{p} 1 *(1-\exp (-\operatorname{lambda} 1 * \mathrm{t}))+\operatorname{alpha} * \mathrm{p} 2 *(1-\exp (-\operatorname{lambda} 2 * \mathrm{t}))+$ alpha $*$ $\mathrm{p} 3 *(1-\exp (-\operatorname{lambda} 3 * \mathrm{t}))$

$20 \operatorname{hpar} 5<-\mathrm{p} 1 * \exp (-\operatorname{lambda} 1 * \mathrm{t})+\mathrm{p} 2 * \exp (-\operatorname{lambda} 2 * \mathrm{t})+\mathrm{p} 3 * \exp (-\operatorname{lambda} 3 * \mathrm{t})$

21 Gerar um vetor multinomial V de parâmetros (N,(hpar1, hpar2, hpar3, hpar4, $\operatorname{hpar} 5)$ )

22 if $\mathrm{V}=(\mathrm{x} 1, \mathrm{x} 2, \mathrm{x} 3, \mathrm{z}, \mathrm{m})$

$23 \quad \mathrm{i}<-\mathrm{i}+1$

24

i-ésima observação aceita <- (alpha,p1,p2,p3, lambda1, lambda2, lambda3)

end if

end while

\subsubsection{Exemplo numérico para $N$ pequeno}

Vamos considerar que 8 peças foram submetidas ao processo de estresse de duração de 10 unidades de tempo. Após o término do teste, foram obtidos os seguintes valores: $x_{1}=3, x_{2}=1$, $x_{3}=2, z=1$ e $m=1$, ou seja, $D=(3,1,2,1,1)$. Por meio do algoritmo Rejeição 1 em ESS para 3 tipos de peças, obtemos uma amostra de 10.000 observações da distribuição a posteriori do vetor $\left(\alpha, p_{1}, p_{2}, p_{3}, \lambda_{1}, \lambda_{2}, \lambda_{3}\right)$. Utilizamos os valores $\alpha_{1}=3, \alpha_{2}=2, \alpha_{3}=1, \theta_{1}=2, \theta_{2}=3, \theta_{3}=4 \mathrm{e}$ $t=10$.

A figura 5.1 apresenta os histogramas dos valores simulados de $\alpha, p_{1}, p_{2}, p_{3}=1-p_{1}-p_{2}, \lambda_{1}$, $\lambda_{2}$ e $\lambda_{3}$.

\subsubsection{Comparação com distribuições exatas}

Na seção 4.1.1, utilizamos a expressão 3.7 para obter os núcleos das distribuições marginais unidimensionais a posteriori de $\alpha, p, \lambda_{b}$ e $\lambda_{r}$. Utilizando o mesmo raciocínio, iremos obter, a partir de 5.5, as expressões das posterioris marginais unidimensionais de $\alpha, p_{1}, p_{2}, p_{3}, \lambda_{1}, \lambda_{2}$ e $\lambda_{3}$, para o caso em que $k=3$. A partir destas expressões, compararemos os resultados obtidos nas simulações com os valores exatos para o caso particular apresentado em 5.2.1.

Primeiramente, iremos encontrar a posteriori de $\alpha$. A partir da expressão 5.5, temos que:

$$
f\left(\alpha \mid x_{1}, x_{2}, x_{3}, z, m\right) \propto \alpha^{z}(1-\alpha)^{\left(x_{1}+x_{2}+x_{3}\right)} \mathbb{1}_{(0 \leq \alpha \leq 1)}
$$

Seguindo o mesmo feito em 4.1.1, comparamos os valores da expressão $5.10 \mathrm{com}$ os valores simulados de acordo com o procedimento descrito em 5.2.1. A figura 5.2 apresenta esta comparação.

Pode-se verificar que a simulação apresentou uma amostra bastante fiel à distribuição exata do parâmetro.

A seguir, as expressões a posteriori de $p_{1}, p_{2}$ e $p_{3}$, bem como as figuras comparativas da distribuição a posteriori com as simulações. 

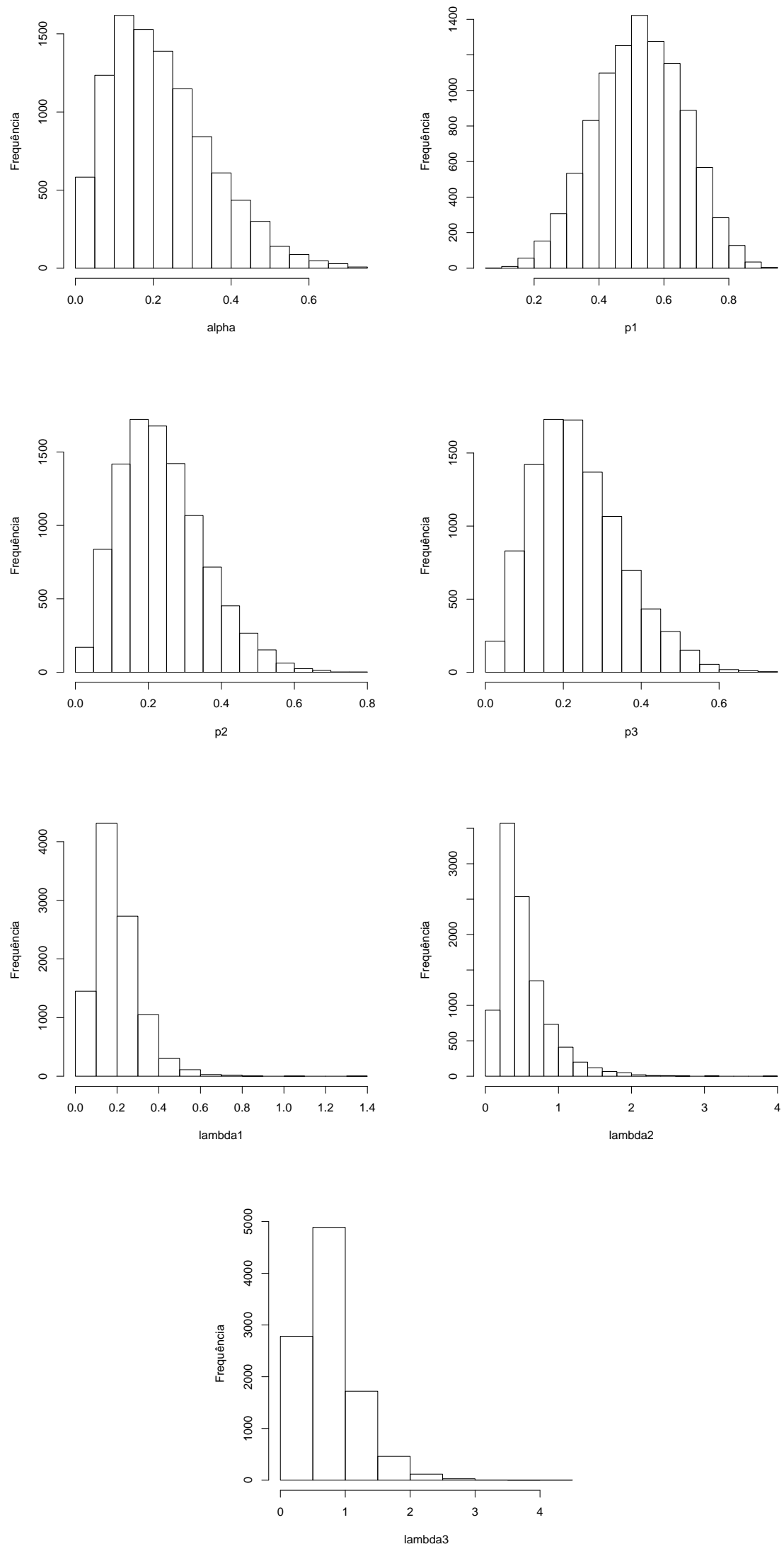

Figura 5.1: Histograma dos valores simulados de $\alpha, p_{1}, p_{2}, p_{3}, \lambda_{1}, \lambda_{2}$ e $\lambda_{3}$. 


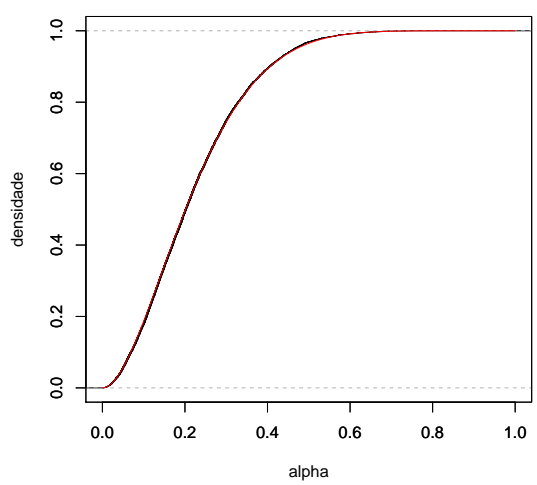

Figura 5.2: Densidade acumulada de $\alpha$ (em vermelho) e densidade simulada (em preto).

$$
f\left(p_{1} \mid x_{1}, x_{2}, x_{3}, z, m\right) \propto
$$

$$
\begin{array}{r}
\propto \sum_{i_{1}=0}^{x_{1}} \sum_{i_{2}=0}^{x_{2}} \sum_{i_{3}=0}^{x_{3}} \sum_{|\beta|=z} \sum_{j_{1}=0}^{\beta_{1}} \sum_{j_{2}=0}^{\beta_{2}} \sum_{j_{3}=0}^{\beta_{3}} \sum_{|\gamma|=m}\left(\begin{array}{c}
x_{1} \\
i_{1}
\end{array}\right)\left(\begin{array}{c}
x_{2} \\
i_{2}
\end{array}\right)\left(\begin{array}{c}
x_{3} \\
i_{3}
\end{array}\right)\left(\begin{array}{c}
z \\
|\beta|
\end{array}\right)\left(\begin{array}{c}
\beta_{1} \\
j_{1}
\end{array}\right)\left(\begin{array}{c}
\beta_{2} \\
j_{2}
\end{array}\right)\left(\begin{array}{c}
\beta_{3} \\
j_{3}
\end{array}\right)\left(\begin{array}{c}
m \\
|\gamma|
\end{array}\right) \\
\frac{p_{1}^{\left(a_{1}+x_{1}+\beta_{1}+\gamma_{1}-1\right)}\left(1-p_{1}\right)^{\left(x_{2}+x_{2}+\beta_{2}+\gamma_{2}+a_{3}+x_{3}+\beta_{3}+\gamma_{3}-1\right)}}{\Gamma\left(a_{1}-j_{1}+x_{2}-i_{2}+\beta_{2}-j_{2}+x_{3}-i_{3}+\beta_{3}-j_{3}\right)} \frac{\Gamma\left(a_{2}+x_{2}+\beta_{2}+\gamma_{2}\right) \Gamma\left(a_{3}+x_{3}+\beta_{3}+\gamma_{3}\right)}{t^{3}\left(\frac{\theta_{3}}{t}+x_{3}-i_{3}+\beta_{3}-j_{3}+\gamma_{3}\right)\left(\frac{\theta_{2}}{t}+x_{2}-i_{2}+\beta_{2}-j_{2}+\gamma_{2}+x_{3}-i_{3}+\beta_{3}-j_{3}+\gamma_{3}\right)} \\
\frac{1}{\left(\frac{\theta_{1}}{t}+x_{1}-i_{1}+\beta_{1}-j_{1}+\gamma_{1}+x_{2}-i_{2}+\beta_{2}-j_{2}+\gamma_{2}+x_{3}-i_{3}+\beta_{3}-j_{3}+\gamma_{3}\right)} \mathbb{1}_{\left(0 \leq p_{1} \leq 1\right)}
\end{array}
$$

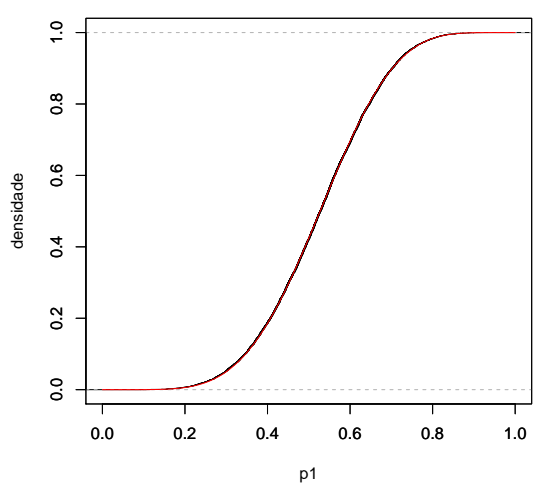

Figura 5.3: Densidade acumulada de $p_{1}$ (em vermelho) e densidade simulada (em preto). 


$$
\begin{array}{r}
\propto \sum_{i_{1}=0}^{x_{1}} \sum_{i_{2}=0}^{x_{2}} \sum_{i_{3}=0}^{x_{3}} \sum_{|\beta|=z} \sum_{j_{1}=0}^{\beta_{1}} \sum_{j_{2}=0}^{\beta_{2}} \sum_{j_{3}=0}^{\beta_{3}} \sum_{|\gamma|=m}\left(\begin{array}{c}
x_{1} \\
i_{1}
\end{array}\right)\left(\begin{array}{c}
x_{2} \\
i_{2}
\end{array}\right)\left(\begin{array}{c}
x_{3} \\
i_{3}
\end{array}\right)\left(\begin{array}{c}
z \\
|\beta|
\end{array}\right)\left(\begin{array}{c}
\beta_{1} \\
j_{1}
\end{array}\right)\left(\begin{array}{c}
\beta_{2} \\
j_{2}
\end{array}\right)\left(\begin{array}{c}
\beta_{3} \\
j_{3}
\end{array}\right)\left(\begin{array}{c}
m \\
|\gamma|
\end{array}\right) \\
\frac{p_{2}^{\left(a_{2}+x_{2}+\beta_{2}+\gamma_{2}-1\right)}\left(1-p_{2}\right)^{\left(x_{1}+i_{1}+x_{1}+\beta_{1}+\gamma_{1}+a_{3}+x_{3}+\beta_{3}+\gamma_{3}-1\right)}}{\Gamma\left(a_{1}+x_{2}-j_{2}+x_{3}-i_{3}+\beta_{3}-j_{3}\right)} \frac{\Gamma\left(a_{1}+x_{1}+\beta_{1}+\gamma_{1}\right) \Gamma\left(a_{3}+x_{3}+\beta_{3}+\gamma_{3}\right)}{t^{3}\left(\frac{\theta_{3}}{t}+x_{3}-i_{3}+\beta_{3}-j_{3}+\gamma_{3}\right)\left(\frac{\theta_{2}}{t}+x_{2}-i_{2}+\beta_{2}-j_{2}+\gamma_{2}+x_{3}-i_{3}+\beta_{3}-j_{3}+\gamma_{3}\right)} \\
\frac{1}{\left(\frac{\theta_{1}}{t}+x_{1}-i_{1}+\beta_{1}-j_{1}+\gamma_{1}+x_{2}-i_{2}+\beta_{2}-j_{2}+\gamma_{2}+x_{3}-i_{3}+\beta_{3}-j_{3}+\gamma_{3}\right)} \mathbb{1}_{\left(0 \leq p_{2} \leq 1\right)}
\end{array}
$$

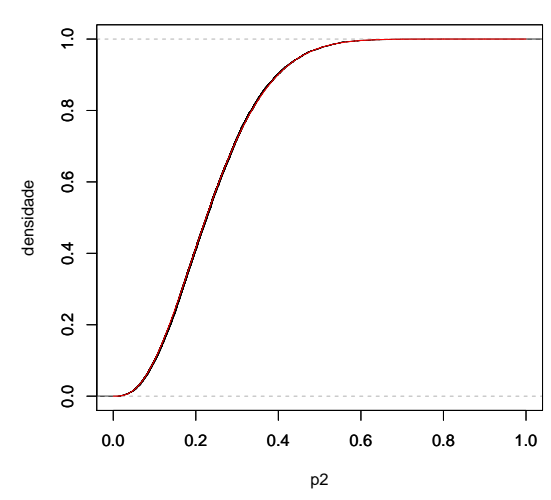

Figura 5.4: Densidade acumulada de $p_{2}$ (em vermelho) e densidade simulada (em preto).

$f\left(p_{3} \mid x_{1}, x_{2}, x_{3}, z, m\right) \propto$

$$
\begin{array}{r}
\propto \sum_{i_{1}=0}^{x_{1}} \sum_{i_{2}=0}^{x_{2}} \sum_{i_{3}=0}^{x_{3}} \sum_{|\beta|=z} \sum_{j_{1}=0}^{\beta_{1}} \sum_{j_{2}=0}^{\beta_{2}} \sum_{j_{3}=0}^{\beta_{3}} \sum_{|\gamma|=m}\left(\begin{array}{c}
x_{1} \\
i_{1}
\end{array}\right)\left(\begin{array}{c}
x_{2} \\
i_{2}
\end{array}\right)\left(\begin{array}{c}
x_{3} \\
i_{3}
\end{array}\right)\left(\begin{array}{c}
z \\
|\beta|
\end{array}\right)\left(\begin{array}{c}
\beta_{1} \\
j_{1}
\end{array}\right)\left(\begin{array}{c}
\beta_{2} \\
j_{2}
\end{array}\right)\left(\begin{array}{c}
\beta_{3} \\
j_{3}
\end{array}\right)\left(\begin{array}{c}
m \\
|\gamma|
\end{array}\right) \\
\frac{p_{3}^{\left(a_{3}+x_{3}+\beta_{3}+\gamma_{3}-1\right)}\left(1-p_{3}\right)^{\left(x_{1}-i_{1}+\beta_{1}-j_{1}+x_{2}-i_{2}+\beta_{2}-j_{2}+x_{3}+x_{3}+a_{2}+x_{2}+\beta_{2}+\gamma_{2}-1\right)}}{\Gamma\left(a_{1}+x_{3}\right)} \frac{\Gamma\left(a_{1}+x_{1}+\beta_{1}+\gamma_{1}\right) \Gamma\left(a_{2}+x_{2}+\beta_{2}+\gamma_{2}\right)}{t^{3}\left(\frac{\theta_{3}}{t}+x_{3}-i_{3}+\beta_{3}-j_{3}+\gamma_{3}\right)\left(\frac{\theta_{2}}{t}+x_{2}-i_{2}+\beta_{2}-j_{2}+\gamma_{2}+x_{3}-i_{3}+\beta_{3}-j_{3}+\gamma_{3}\right)} \\
\frac{1}{\left(\frac{\theta_{1}}{t}+x_{1}-i_{1}+\beta_{1}-j_{1}+\gamma_{1}+x_{2}-i_{2}+\beta_{2}-j_{2}+\gamma_{2}+x_{3}-i_{3}+\beta_{3}-j_{3}+\gamma_{3}\right)} \mathbb{1}_{\left(0 \leq p_{3} \leq 1\right)}
\end{array}
$$

Por fim, as expressões a posteriori de $\lambda_{1}, \lambda_{2}$ e $\lambda_{3}$, bem como as figuras comparativas da distribuição a posteriori com as simulações. 


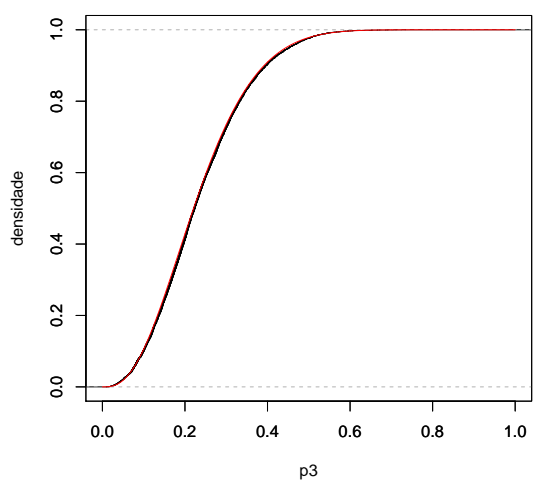

Figura 5.5: Densidade acumulada de $p_{3}$ (em vermelho) e densidade simulada (em preto).

$$
\begin{aligned}
& f\left(\lambda_{1} \mid x_{1}, x_{2}, x_{3}, z, m\right) \propto \\
& \propto \sum_{i_{1}=0}^{x_{1}} \sum_{i_{2}=0}^{x_{2}} \sum_{i_{3}=0}^{x_{3}} \sum_{|\beta|=z} \sum_{j_{1}=0}^{\beta_{1}} \sum_{j_{2}=0}^{\beta_{2}} \sum_{j_{3}=0}^{\beta_{3}} \sum_{|\gamma|=m}\left(\begin{array}{c}
x_{1} \\
i_{1}
\end{array}\right)\left(\begin{array}{c}
x_{2} \\
i_{2}
\end{array}\right)\left(\begin{array}{c}
x_{3} \\
i_{3}
\end{array}\right)\left(\begin{array}{c}
z \\
|\beta|
\end{array}\right)\left(\begin{array}{c}
\beta_{1} \\
j_{1}
\end{array}\right)\left(\begin{array}{c}
\beta_{2} \\
j_{2}
\end{array}\right)\left(\begin{array}{c}
\beta_{3} \\
j_{3}
\end{array}\right)\left(\begin{array}{c}
m \\
|\gamma|
\end{array}\right) \\
& (-1)^{\left(x_{1}-i_{1}+\beta_{1}-j_{1}+x_{2}-i_{2}+\beta_{2}-j_{2}+x_{3}-i_{3}+\beta_{3}-j_{3}\right)} \frac{\Gamma\left(a_{1}+x_{1}+\beta_{1}+\gamma_{1}\right) \Gamma\left(a_{2}+x_{2}+\beta_{2}+\gamma_{2}\right) \Gamma\left(a_{3}+x_{3}+\beta_{3}+\gamma_{3}\right)}{\Gamma\left(a_{1}+x_{1}+\beta_{1}+\gamma_{1}+a_{2}+x_{2}+\beta_{2}+\gamma_{2}+a_{3}+x_{3}+\beta_{3}+\gamma_{3}\right)} \\
& e^{-\lambda_{1} t\left(\frac{\theta_{1}}{t}+x_{1}-i_{1}+\beta_{1}-j_{1}+\gamma_{1}+x_{2}-i_{2}+\beta_{2}-j_{2}+\gamma_{2}+x_{3}-i_{3}+\beta_{3}-j_{3}+\gamma_{3}\right)} \\
& \overline{t^{2}\left(\frac{\theta_{3}}{t}+x_{3}-i_{3}+\beta_{3}-j_{3}+\gamma_{3}\right)\left(\frac{\theta_{2}}{t}+x_{2}-i_{2}+\beta_{2}-j_{2}+\gamma_{2}+x_{3}-i_{3}+\beta_{3}-j_{3}+\gamma_{3}\right)} \\
& \mathbb{1}_{\left(\lambda_{1}>0\right)}
\end{aligned}
$$

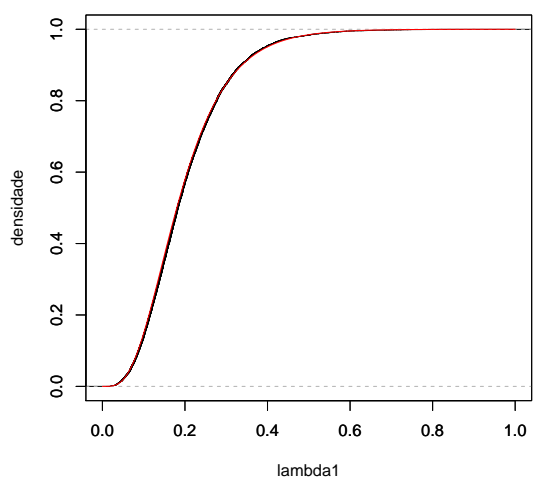

Figura 5.6: Densidade acumulada de $\lambda_{1}$ (em vermelho) e densidade simulada (em preto). 


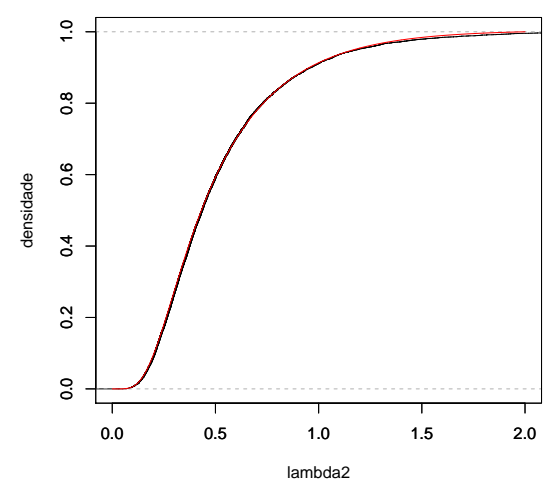

Figura 5.7: Densidade acumulada de $\lambda_{2}$ (em vermelho) e densidade simulada (em preto).

$$
\begin{array}{r}
\propto \sum_{i_{1}=0}^{x_{1}} \sum_{i_{2}=0}^{x_{2}} \sum_{i_{3}=0}^{x_{3}} \sum_{|\beta|=z} \sum_{j_{1}=0}^{\beta_{1}} \sum_{j_{2}=0}^{\beta_{2}} \sum_{j_{3}=0}^{\beta_{3}} \sum_{|\gamma|=m}\left(\begin{array}{c}
x_{1} \\
i_{1}
\end{array}\right)\left(\begin{array}{c}
x_{2} \\
i_{2}
\end{array}\right)\left(\begin{array}{c}
x_{3} \\
i_{3}
\end{array}\right)\left(\begin{array}{c}
z \\
|\beta|
\end{array}\right)\left(\begin{array}{c}
\beta_{1} \\
j_{1}
\end{array}\right)\left(\begin{array}{c}
\beta_{2} \\
j_{2}
\end{array}\right)\left(\begin{array}{c}
\beta_{3} \\
j_{3}
\end{array}\right)\left(\begin{array}{c}
m \\
|\gamma|
\end{array}\right) \\
(-1)^{\left(x_{1}-i_{1}+\beta_{1}-j_{1}+x_{2}-i_{2}+\beta_{2}-j_{2}+x_{3}-i_{3}+\beta_{3}-j_{3}\right)} \frac{\Gamma\left(a_{1}+x_{1}+\beta_{1}+\gamma_{1}\right) \Gamma\left(a_{2}+x_{2}+\beta_{2}+\gamma_{2}\right) \Gamma\left(a_{3}+x_{3}+\beta_{3}+\gamma_{3}\right)}{\Gamma\left(a_{1}+x_{1}+\beta_{1}+\gamma_{1}+a_{2}+x_{2}+\beta_{2}+\gamma_{2}+a_{3}+x_{3}+\beta_{3}+\gamma_{3}\right)} \\
\frac{e^{-\lambda_{3} t\left(\frac{\theta_{3}}{t}+x_{3}-i_{3}+\beta_{3}-j_{3}+\gamma_{3}\right)}}{t\left(\frac{\left(\theta_{1}-\theta_{2}\right)}{t}+x_{1}-i_{1}+\beta_{1}-j_{1}+\gamma_{1}\right)} \\
{\left[\frac{\left(1-e^{-\lambda_{3} t\left(\frac{\left(\theta_{2}-\theta_{3}\right)}{t}+x_{2}-i_{2}+\beta_{2}-j_{2}+\gamma_{2}\right)}\right)}{t\left(\frac{\left(\theta_{2}-\theta_{3}\right)}{t}+x_{2}-i_{2}+\beta_{2}-j_{2}+\gamma_{2}\right)}-\frac{\left(1-e^{-\lambda_{3} t\left(\frac{\left(\theta_{1}-\theta_{3}\right)}{t}+x_{1}-i_{1}+\beta_{1}-j_{1}+\gamma_{1}+x_{2}-i_{2}+\beta_{2}-j_{2}+\gamma_{2}\right)}\right)}{t\left(\frac{\left(\theta_{1}-\theta_{3}\right)}{t}+x_{1}-i_{1}+\beta_{1}-j_{1}+\gamma_{1}+x_{2}-i_{2}+\beta_{2}-j_{2}+\gamma_{2}\right)}\right]}
\end{array}
$$




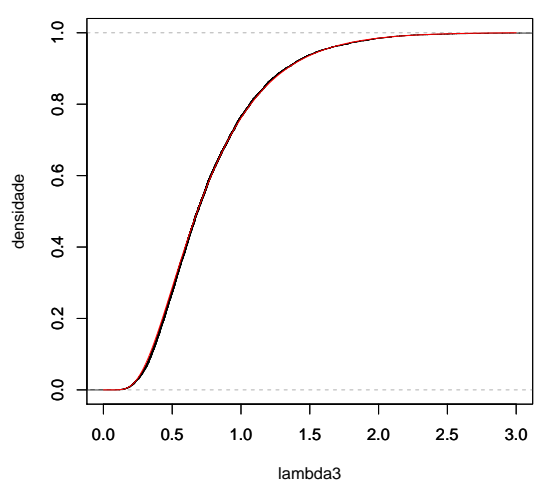

Figura 5.8: Densidade acumulada de $\lambda_{3}$ (em vermelho) e densidade simulada (em preto).

A demonstração de como foram obtidas as expressões 5.10, 5.11, 5.12, 5.13, 5.14, 5.15 e 5.16 podem ser encontradas no apêndice D. A partir das figuras 5.2, 5.3, 5.4, 5.5, 5.6, 5.7 e 5.8, pode-se verificar a boa aderência da simulação via algoritmo Rejeição 1, comparativamente à distribuição exata a posteriori dos parâmetros.

\subsubsection{Algoritmo Rejeição 2}

Assim como feito em 4.1.2, iremos utilizar o algoritmo Rejeição 2 para verificar a influência do limitador $\varepsilon$ na simulação da posteriori dos parâmetros. Agora, temos que $\mathbb{T}_{N}^{5}=\left\{\left(x_{1}, x_{2}, x_{3}, z, m\right) \in\right.$ $\left.\mathbb{N}^{5}: x_{1}+x_{2}+x_{3}+z+m=N\right\}$, que possui $\left(\begin{array}{c}N+4 \\ 4\end{array}\right)=(N+4)(N+3)(N+2)(N+1) / 24$ elementos. Para $N=8$, temos que $\left|\mathbb{T}_{8}^{5}\right|=495$.

A figura 5.9 apresenta o histograma da distância euclidiana entre cada elemento de $\mathbb{T}_{8}^{5}$ e o vetor observado $(3,1,2,1,1)$.

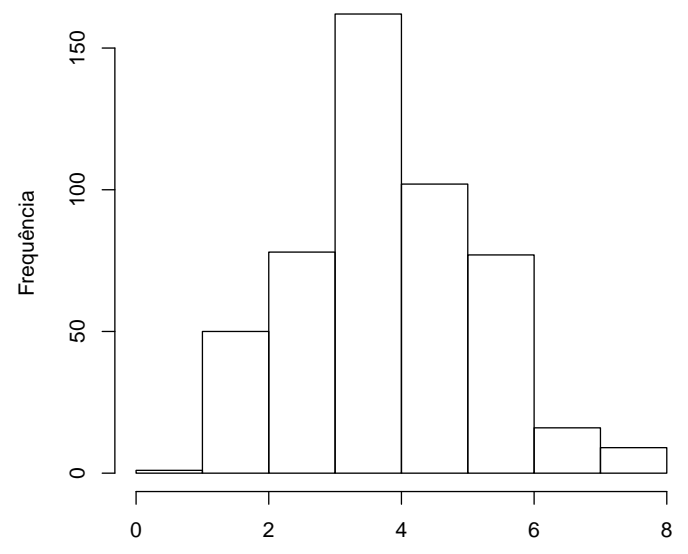

Figura 5.9: Histograma da distância euclidiana entre os elementos de $\mathbb{T}_{8}^{5}$ e $(3,1,2,1,1)$

A figura 5.10 apresenta as densidades simuladas dos parâmetros, variando-se o valor de $\varepsilon$.

Assim como em 4.1.2, vemos que quanto menor o valor de $\varepsilon$, mais a densidade simulada se aproxima da posteriori exata. Verificamos que, para $\lambda_{1}, \lambda_{2}$ e $\lambda_{3}$, quando $\varepsilon \geq 2$ o valor de $\varepsilon$ parece 

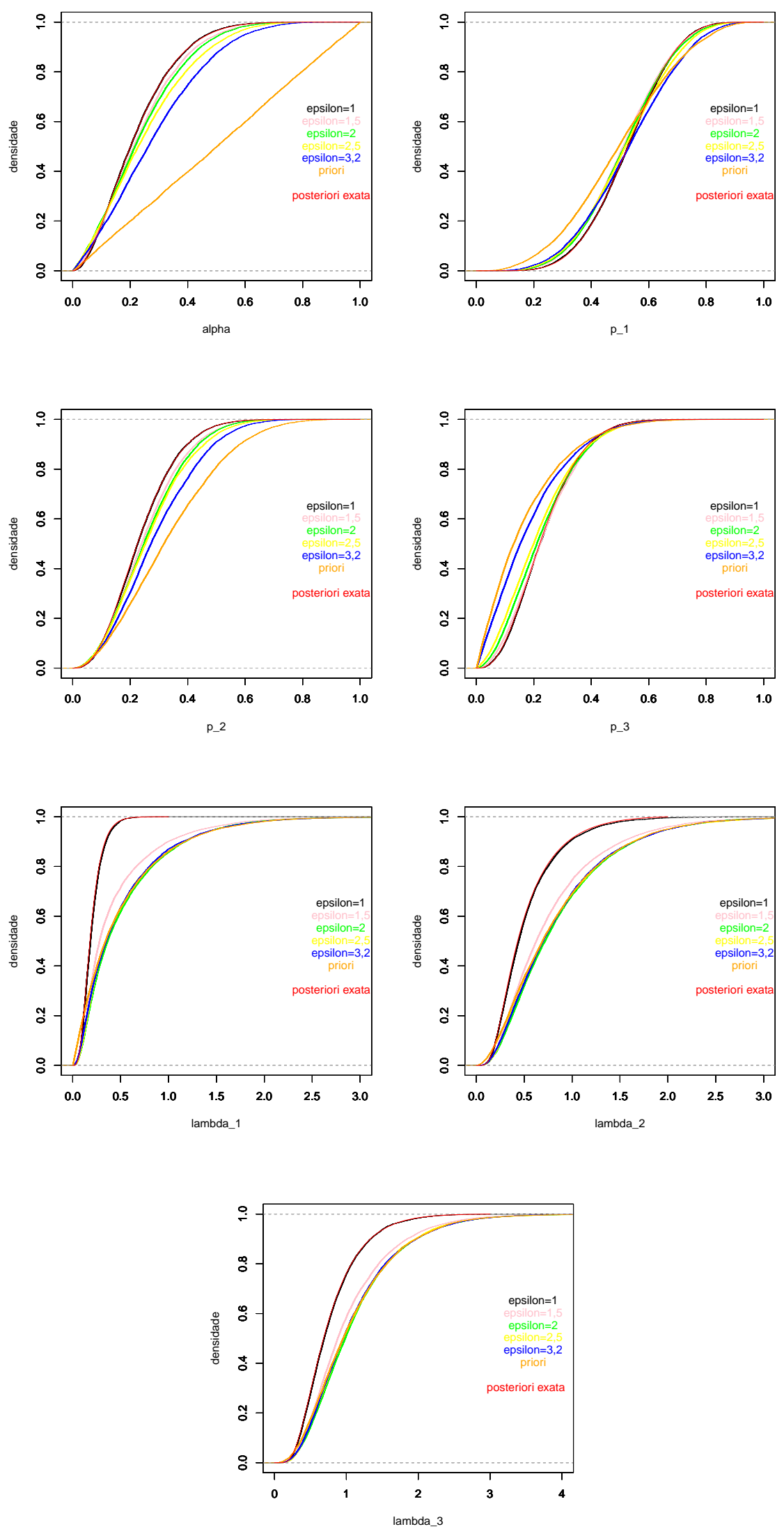

Figura 5.10: Densidades simuladas de $\alpha, p_{1}, p_{2}, p_{3}, \lambda_{1}, \lambda_{2}$ e $\lambda_{3}$ para diferentes valores de $\varepsilon$ 
ter pouca influência na densidade simulada, gerando valores próximos à priori. Quando $\varepsilon=1,5$ (curva rosa), a densidade simulada se aproxima mais da posteriori, bem como quando $\varepsilon=1$, que é o caso em que simulamos exatamente a posteriori, conforme a figura 5.9.

\subsection{Exemplos numéricos para $N$ grande}

Mantendo as distribuições a priori apresentadas em 5.2.1, podemos utilizar a expressão 5.8 para encontrar o tempo $t$ que minimiza o custo esperado por peça a priori. Aqui, simulamos valores das prioris e, por meio do método de Monte Carlo, calculamos o custo esperado para cada tempo $t$ entre 0 e 30 unidades. Para isto, vamos considerar que $c_{1}=0,01, c_{2}=40, c_{3}=100, d_{1}=10, d_{2}=1$, $d_{3}=0,01, e_{1}=0,01$ e $e_{2}=1$. O gráfico 5.11 apresenta o custo esperado, a priori, para cada tempo $t$.

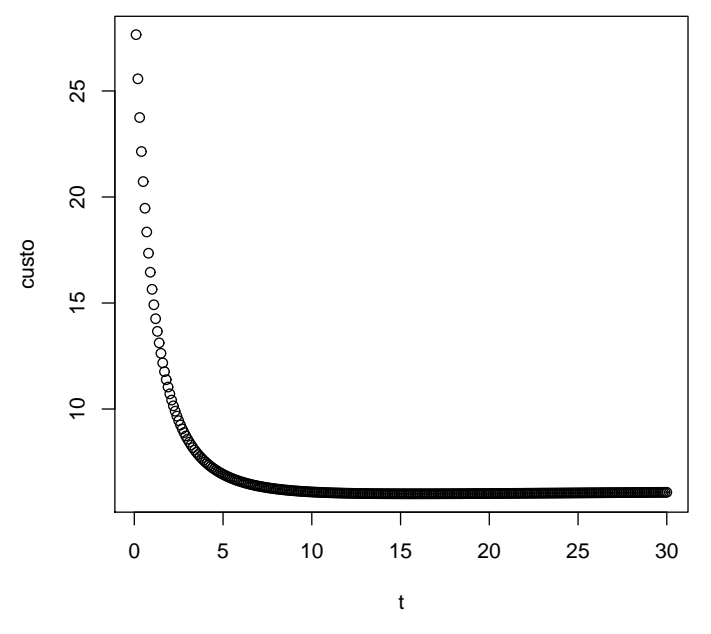

Figura 5.11: Função de risco a priori

O valor de $t$ que minimiza a função apresentada na figura 5.11 é 15,8 unidades de tempo.

Vamos utilizar o algoritmo Rejeição 2 para simular 10.000 valores da distribuição condicional de $\left(\alpha, p_{1}, p_{2}, p_{3}, \lambda_{1}, \lambda_{2}, \lambda_{3} \mid x_{1}, x_{2}, x_{3}, z, m\right)$. Para este exemplo, consideramos um lote de $N=1000$ peças, que foram submetidas ao procedimento de estresse durante 15,8 unidades de tempo. Após o estresse, observou-se que 470 peças falharam e foram diagnosticadas como de qualidade 1, 280 falharam e foram diagnosticadas como de qualidade 2, 20 falharam e foram diagnosticadas como de qualidade 3, 220 falharam no estresse e não foi possível identificar sua qualidade, e 10 peças sobreviveram ao estresse. Ou seja, $D=\left(x_{1}, x_{2}, x_{3}, z, m\right)=(470,280,20,220,10)$. Na figura 5.12, encontramos os histogramas dos valores simulados dos parâmetros, a posteriori. 

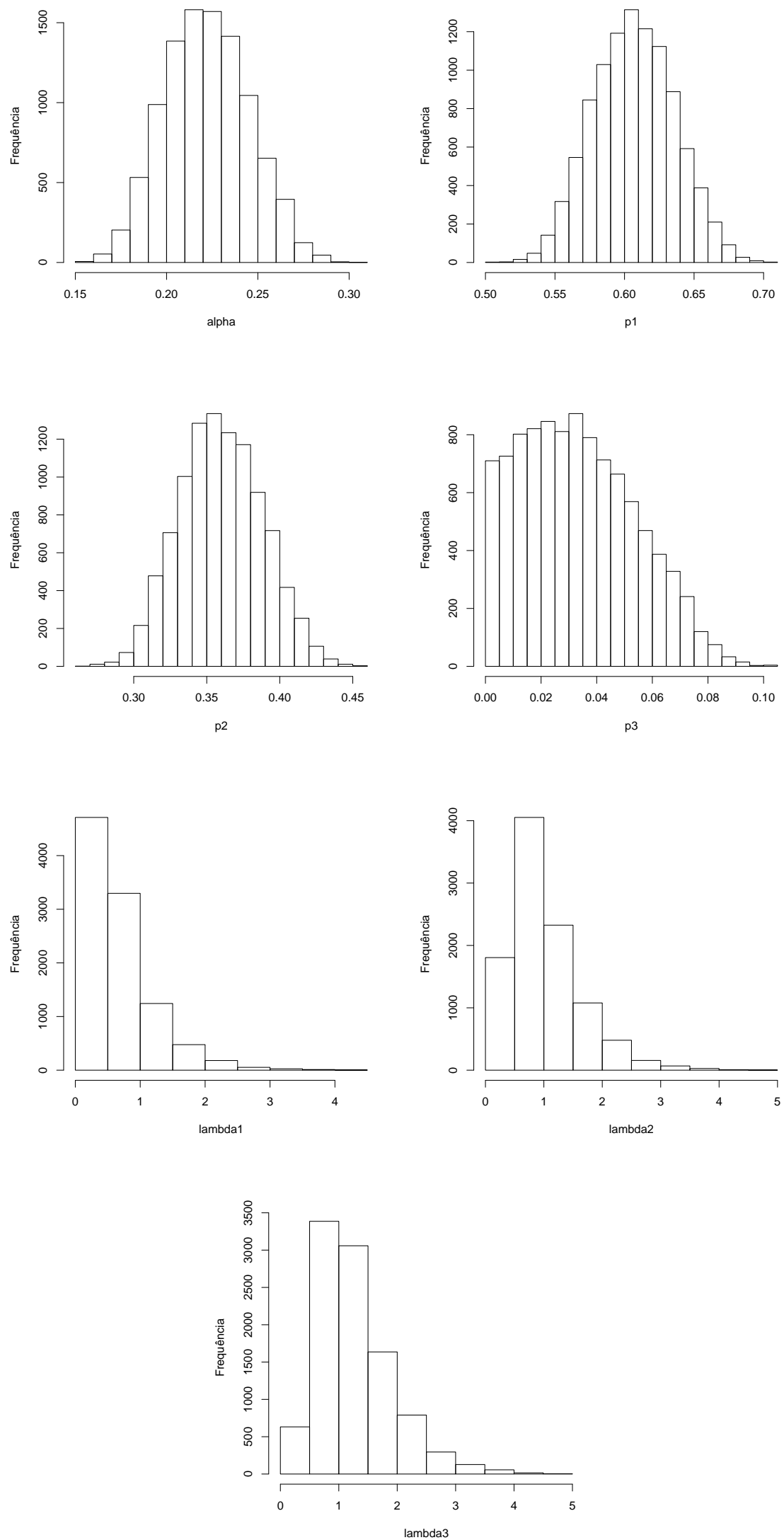

Figura 5.12: Histograma dos valores simulados de $\alpha, p_{1}, p_{2}, p_{3}, \lambda_{1}, \lambda_{2}$ e $\lambda_{3}$. 


\subsubsection{Função de custo}

Na figura 5.13, obtemos o gráfico da função de custo a posteriori dados os valores simulados no início da seção 5.3 .

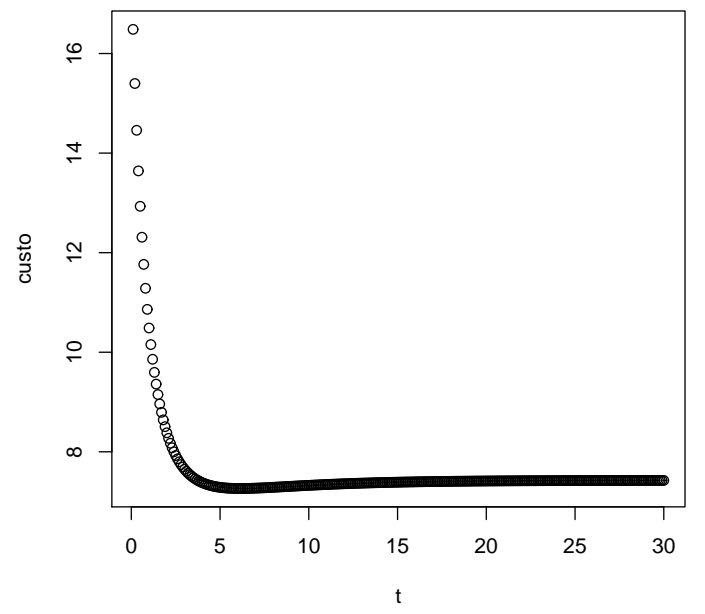

Figura 5.13: Função de risco a posteriori

O tempo ótimo obtido para a duração do estresse, a posteriori, é de 6,1 unidades de tempo. Portanto, a partir da informação amostral, obtém-se uma redução de 61,3\% no tempo de duração do teste de estresse. 


\section{Capítulo 6}

\section{Conclusões}

Neste trabalho, revisamos o conceito de algoritmos ABC (approximate Bayesian computation), motivando sua utilização em situações em que a verossimilhança (e a posteriori) são intratáveis analiticamente. Detalhamos os algoritmos básicos Rejeição 1 - no qual se obtém uma amostra exata da posteriori - e Rejeição 2 - no qual se obtém uma amostra aproximada da posteriori. Outros algoritmos $\mathrm{ABC}$ mais avançados foram brevemente descritos.

Exploramos o uso do ABC no contexto de Environmental Stress Screening (ESS), procedimento aplicado na indústria para deteç̧ão de peças defeituosas antes que sejam utilizadas no produto final. Por meio de uma quantificação proposta por Barlow et al. (1994), aplicamos os algoritmos Rejeição 1 (para uma quantidade pequena de peças) e Rejeição 2 (para uma quantidade qualquer de peças) no cenário em que existem peças de dois tipos: boas ou ruins.

Para uma quantidade pequena de peças, foi possível verificar que as distribuições a posteriori simuladas pelo algoritmo Rejeição 1 eram idênticas às posterioris exatas de cada um dos parâmetros. Por meio do algoritmo Rejeição 2 observamos a influência de $\varepsilon$ na qualidade das distribuições geradas.

Para um número grande de peças, utilizamos o algoritmo Rejeição 2 para obter observações simuladas das distribuições a posteriori dos parâmetros. A partir das observações simuladas, foi possível estimar o tempo ótimo de duração para um futuro teste de estresse que minimize o custo do teste por peça.

Propusemos, por fim, uma generalização do problema de ESS no qual existem $k$ tipos de peças, que se diferenciam pelo seu nível de qualidade. Propondo uma estrutura de custos, foi possível novamente estimar o tempo ótimo de duração de um futuro teste de estresse, a partir do algoritmo Rejeição 2.

\subsection{Sugestões para pesquisas futuras}

- Estudar variações para a função de distância utilizada na aplicação do ABC

- Verificar a eficiência de algoritmos ABC mais sofisticados na duração da simulação no contexto do ESS

- Propor uma quantificação do ESS na qual a taxa de falha é variável ao longo do tempo (possivelmente não-decrescente) para o mesmo tipo de peça

- Propor uma quantificação do ESS na qual erros de diagnóstico a respeito da qualidade das peças sejam possíveis 
- Estudar os possíveis impactos relacionados à ausência de identificabilidade do modelo, bem como possíveis restrições adicionais que garantam tal propriedade 


\section{Apêndice A}

\section{Desenvolvimento da Expressão 3.7}

$$
\begin{aligned}
& f\left(\alpha, p, \lambda_{b}, \lambda_{r} \mid x, y, z, m\right) \propto f\left(p, \lambda_{b}, \lambda_{r}\right) L\left(\alpha, p, \lambda_{b}, \lambda_{r} \mid x, y, z, m\right) \mathbb{1}_{(0 \leq \alpha \leq 1)} \propto \\
& \propto \theta \tau \frac{\Gamma(a+b)}{\Gamma(a) \Gamma(b)} p^{a-1}(1-p)^{b-1} e^{-\lambda_{b}(\theta-\tau)} e^{-\lambda_{r} \tau} \\
& {\left[(1-\alpha) p\left(1-e^{-\lambda_{r} t}\right)\right]^{x}\left[(1-\alpha)(1-p)\left(1-e^{-\lambda_{b} t}\right)\right]^{y}} \\
& {\left[\alpha p\left(1-e^{-\lambda_{r} t}\right)+\alpha(1-p)\left(1-e^{-\lambda_{b} t}\right)\right]^{z}\left[p e^{-\lambda_{r} t}+(1-p) e^{-\lambda_{b} t}\right]^{m}} \\
& \mathbb{1}_{(0 \leq \alpha \leq 1)} \mathbb{1}_{(0 \leq p \leq 1)} \mathbb{1}_{\left(\lambda_{r}>\lambda_{b}>0\right)} \propto \\
& \propto p^{a-1}(1-p)^{b-1} e^{-\lambda_{b}(\theta-\tau)} e^{-\lambda_{r} \tau}(1-\alpha)^{x} p^{x}\left(1-e^{-\lambda_{r} t}\right)^{x}(1-\alpha)^{y}(1-p)^{y}\left(1-e^{-\lambda_{b} t}\right)^{y} \\
& \alpha^{z}\left\{\sum_{k=0}^{z}\left(\begin{array}{l}
z \\
k
\end{array}\right)\left[p\left(1-e^{-\lambda_{r} t}\right)\right]^{k}\left[(1-p)\left(1-e^{-\lambda_{b} t}\right)\right]^{z-k}\right\}\left\{\sum_{l=0}^{m}\left(\begin{array}{c}
m \\
l
\end{array}\right)\left[p e^{-\lambda_{r} t}\right]^{l}\left[(1-p) e^{-\lambda_{b} t}\right]^{m-l}\right\} \\
& \mathbb{1}_{(0 \leq \alpha \leq 1)} \mathbb{1}_{(0 \leq p \leq 1)} \mathbb{1}_{\left(\lambda_{r}>\lambda_{b}>0\right)} \propto \\
& \propto \alpha^{z}(1-\alpha)^{x+y} p^{a+x-1}(1-p)^{b+y-1} e^{-\lambda_{b}(\theta-\tau)} e^{-\lambda_{r} \tau} \\
& \sum_{i=0}^{x}\left(\begin{array}{l}
x \\
i
\end{array}\right) 1^{i}\left(-e^{-\lambda_{r} t}\right)^{x-i} \sum_{j=0}^{y}\left(\begin{array}{c}
y \\
j
\end{array}\right) 1^{j}\left(-e^{-\lambda_{b} t}\right)^{y-j} \\
& \sum_{k=0}^{z}\left(\begin{array}{l}
z \\
k
\end{array}\right) p^{k}\left[\sum_{r=0}^{k}\left(\begin{array}{l}
k \\
r
\end{array}\right) 1^{r}\left(-e^{-\lambda_{r} t}\right)^{k-r}\right](1-p)^{z-k}\left[\sum_{s=0}^{z-k}\left(\begin{array}{c}
z-k \\
s
\end{array}\right) 1^{s}\left(-e^{-\lambda_{b} t}\right)^{z-k-s}\right] \\
& \sum_{l=0}^{m}\left(\begin{array}{c}
m \\
l
\end{array}\right) p^{l}\left(e^{-\lambda_{r} t}\right)^{l}(1-p)^{m-l}\left(e^{-\lambda_{b} t}\right)^{m-l} \\
& \mathbb{1}_{(0 \leq \alpha \leq 1)} \mathbb{1}_{(0 \leq p \leq 1)} \mathbb{1}_{\left(\lambda_{r}>\lambda_{b}>0\right)} \propto \\
& \propto \alpha^{z}(1-\alpha)^{x+y} \sum_{i=0}^{x} \sum_{j=0}^{y} \sum_{l=0}^{m} \sum_{k=0}^{z} \sum_{r=0}^{k} \sum_{s=0}^{z-k}\left(\begin{array}{l}
x \\
i
\end{array}\right)\left(\begin{array}{l}
y \\
j
\end{array}\right)\left(\begin{array}{c}
m \\
l
\end{array}\right)\left(\begin{array}{l}
z \\
k
\end{array}\right)\left(\begin{array}{c}
k \\
r
\end{array}\right)\left(\begin{array}{c}
z-k \\
s
\end{array}\right)(-1)^{x+y+z-i-j-r-s} \\
& p^{a+x+l+k-1}(1-p)^{b+y+z+m-l-k-1} e^{-\lambda_{r} t\left(\frac{\tau}{t}+x-i+k-r+l\right)} e^{-\lambda_{b} t\left(\frac{(\theta-\tau)}{t}+y-j+z-k-s+m-l\right)} \\
& \mathbb{1}_{(0 \leq \alpha \leq 1)} \mathbb{1}_{(0 \leq p \leq 1)} \mathbb{1}_{\left(\lambda_{r}>\lambda_{b}>0\right)}
\end{aligned}
$$


APÊNDICE A 


\section{Apêndice B}

\section{Posterioris marginais de $\alpha, p, \lambda_{b}$ e $\lambda_{r}$ para 2 tipos de peças}

\section{B.1 Posteriori marginal de $\alpha$}

A partir da expressão 3.7, obtemos a distribuição marginal de $\alpha$. Sabemos que vale:

$$
f\left(\alpha, p, \lambda_{b}, \lambda_{r} \mid x, y, z, m\right) \propto
$$

$$
\begin{array}{r}
\propto \alpha^{z}(1-\alpha)^{x+y} \sum_{i=0}^{x} \sum_{j=0}^{y} \sum_{l=0}^{m} \sum_{k=0}^{z} \sum_{r=0}^{k} \sum_{s=0}^{z-k}\left(\begin{array}{c}
x \\
i
\end{array}\right)\left(\begin{array}{c}
y \\
j
\end{array}\right)\left(\begin{array}{c}
m \\
l
\end{array}\right)\left(\begin{array}{c}
z \\
k
\end{array}\right)\left(\begin{array}{c}
k \\
r
\end{array}\right)\left(\begin{array}{c}
z-k \\
s
\end{array}\right)(-1)^{x+y+z-i-j-r-s} \\
p^{a+x+l+k-1}(1-p)^{b+y+z+m-l-k-1} e^{-\lambda_{r} t\left(\frac{\tau}{t}+x-i+k-r+l\right)} e^{-\lambda_{b} t\left(\frac{(\theta-\tau)}{t}+y-j+z-k-s+m-l\right)} \\
\mathbb{1}_{(0 \leq \alpha \leq 1)} \mathbb{1}_{(0 \leq p \leq 1)} \mathbb{1}_{\left(\lambda_{r}>\lambda_{b}>0\right)}= \\
=\alpha^{z}(1-\alpha)^{x+y} g\left(p, \lambda_{b}, \lambda_{r}\right) \mathbb{1}_{(0 \leq \alpha \leq 1)} .
\end{array}
$$

Temos que $g\left(p, \lambda_{b}, \lambda_{r}\right)$ não depende de $\alpha$ e, portanto,

$$
\begin{array}{r}
f(\alpha \mid x, y, z, m) \propto \\
\propto \alpha^{z}(1-\alpha)^{x+y} \int_{0}^{1} \int_{0}^{\infty} \int_{\lambda_{b}}^{\infty} g\left(p, \lambda_{b}, \lambda_{r}\right) \mathbf{d} \lambda_{r} \mathbf{d} \lambda_{b} \mathbf{d} p \mathbb{1}_{(0 \leq \alpha \leq 1)} \propto \\
\propto \mathbf{K} \alpha^{z}(1-\alpha)^{x+y} \mathbb{1}_{(0 \leq \alpha \leq 1)} \\
\propto \alpha^{z}(1-\alpha)^{x+y} \mathbb{1}_{(0 \leq \alpha \leq 1)}
\end{array}
$$




\section{B.2 Posteriori marginal de $p$}

Temos que:

$$
\begin{array}{r}
f\left(p, \lambda_{b}, \lambda_{r} \mid x, y, z, m\right) \propto \int_{0}^{1} \alpha^{z}(1-\alpha)^{x+y} g\left(p, \lambda_{b}, \lambda_{r}\right) \mathbf{d} \alpha \mathbb{1}_{(0 \leq p \leq 1)} \mathbb{1}_{\left(\lambda_{r}>\lambda_{b}>0\right)} \propto \\
\propto g\left(p, \lambda_{b}, \lambda_{r}\right) \frac{\Gamma(z+1) \Gamma(x+y+1)}{\Gamma(x+y+z+2)} \mathbb{1}_{(0 \leq p \leq 1)} \mathbb{1}_{\left(\lambda_{r}>\lambda_{b}>0\right)} \propto \\
\propto g\left(p, \lambda_{b}, \lambda_{r}\right) \mathbb{1}_{(0 \leq p \leq 1)} \mathbb{1}_{\left(\lambda_{r}>\lambda_{b}>0\right)}
\end{array}
$$

Na expressão B.3, temos que $g\left(p, \lambda_{b}, \lambda_{r}\right)$ é dada pelos termos de B.1 que não dependem de $\alpha$. Podemos concluir, então, que $\alpha$ não influi nas posterioris marginais e conjuntas relacionadas a $\left(p, \lambda_{b}, \lambda_{r}\right)$. A partir de B.3, temos: 


$$
\int_{0}^{\infty} \int_{\lambda_{b}}^{\infty} \sum_{i=0}^{x} \sum_{j=0}^{y} \sum_{l=0}^{m} \sum_{k=0}^{z} \sum_{r=0}^{k} \sum_{s=0}^{z-k}\left(\begin{array}{l}
x \\
i
\end{array}\right)\left(\begin{array}{l}
y \\
j
\end{array}\right)\left(\begin{array}{l}
m \\
l
\end{array}\right)\left(\begin{array}{l}
z \\
k
\end{array}\right)\left(\begin{array}{l}
k \\
r
\end{array}\right)\left(\begin{array}{c}
z-k \\
s
\end{array}\right)
$$

$(-1)^{x+y+z-i-j-r-s} p^{a+x+l+k-1}(1-p)^{b+y+z+m-l-k-1} e^{-\lambda_{r} t\left(\frac{\tau}{t}+x-i+k-r+l\right)} e^{-\lambda_{b} t\left(\frac{(\theta-\tau)}{t}+y-j+z-k-s+m-l\right)}$

$$
\mathbf{d} \lambda_{r} \mathbf{d} \lambda_{b} \mathbb{1}_{(0 \leq p \leq 1)} \propto
$$

$$
\begin{aligned}
& \propto \sum_{i=0}^{x} \sum_{j=0}^{y} \sum_{l=0}^{m} \sum_{k=0}^{z} \sum_{r=0}^{k} \sum_{s=0}^{z-k}\left(\begin{array}{l}
x \\
i
\end{array}\right)\left(\begin{array}{l}
y \\
j
\end{array}\right)\left(\begin{array}{l}
m \\
l
\end{array}\right)\left(\begin{array}{l}
z \\
k
\end{array}\right)\left(\begin{array}{l}
k \\
r
\end{array}\right)\left(\begin{array}{c}
z-k \\
s
\end{array}\right)(-1)^{x+y+z-i-j-r-s} \\
& p^{a+x+l+k-1}(1-p)^{b+y+z+m-l-k-1} \\
& \int_{0}^{\infty} e^{-\lambda_{b} t\left(\frac{(\theta-\tau)}{t}+y-j+z-k-s+m-l\right)} \int_{\lambda_{b}}^{\infty} e^{-\lambda_{r} t\left(\frac{\tau}{t}+x-i+k-r+l\right)} \mathbf{d} \lambda_{r} \mathbf{d} \lambda_{b} \mathbb{1}_{(0 \leq p \leq 1)} \propto \\
& \propto \sum_{i=0}^{x} \sum_{j=0}^{y} \sum_{l=0}^{m} \sum_{k=0}^{z} \sum_{r=0}^{k} \sum_{s=0}^{z-k}\left(\begin{array}{l}
x \\
i
\end{array}\right)\left(\begin{array}{l}
y \\
j
\end{array}\right)\left(\begin{array}{l}
m \\
l
\end{array}\right)\left(\begin{array}{l}
z \\
k
\end{array}\right)\left(\begin{array}{l}
k \\
r
\end{array}\right)\left(\begin{array}{c}
z-k \\
s
\end{array}\right)(-1)^{x+y+z-i-j-r-s} \\
& p^{a+x+l+k-1}(1-p)^{b+y+z+m-l-k-1} \\
& \int_{0}^{\infty} e^{-\lambda_{b} t\left(\frac{(\theta-\tau)}{t}+y-j+z-k-s+m-l\right)} \frac{e^{-\lambda_{b} t\left(\frac{\tau}{t}+x-i+k-r+l\right)}}{\tau+t(x-i+k-r+l)} \mathbf{d} \lambda_{b} \mathbb{1}_{(0 \leq p \leq 1)} \propto \\
& \propto \sum_{i=0}^{x} \sum_{j=0}^{y} \sum_{l=0}^{m} \sum_{k=0}^{z} \sum_{r=0}^{k} \sum_{s=0}^{z-k}\left(\begin{array}{l}
x \\
i
\end{array}\right)\left(\begin{array}{l}
y \\
j
\end{array}\right)\left(\begin{array}{l}
m \\
l
\end{array}\right)\left(\begin{array}{l}
z \\
k
\end{array}\right)\left(\begin{array}{l}
k \\
r
\end{array}\right)\left(\begin{array}{c}
z-k \\
s
\end{array}\right)(-1)^{x+y+z-i-j-r-s} \\
& p^{a+x+l+k-1}(1-p)^{b+y+z+m-l-k-1} \\
& \frac{1}{\tau+t(x-i+k-r+l)}(-1) \frac{0-1}{\theta+t(x-i+y-j+z-s-r+m)} \mathbb{1}_{(0 \leq p \leq 1)} \propto \\
& \propto \sum_{i=0}^{x} \sum_{j=0}^{y} \sum_{l=0}^{m} \sum_{k=0}^{z} \sum_{r=0}^{k} \sum_{s=0}^{z-k}\left(\begin{array}{l}
x \\
i
\end{array}\right)\left(\begin{array}{l}
y \\
j
\end{array}\right)\left(\begin{array}{l}
m \\
l
\end{array}\right)\left(\begin{array}{l}
z \\
k
\end{array}\right)\left(\begin{array}{l}
k \\
r
\end{array}\right)\left(\begin{array}{c}
z-k \\
s
\end{array}\right)(-1)^{x+y+z-i-j-r-s} \\
& \frac{p^{a+x+l+k-1}(1-p)^{b+y+z+m-l-k-1}}{[\tau+t(x-i+k-r+l)][\theta+t(x-i+y-j+z-s-r+m)]} \mathbb{1}_{(0 \leq p \leq 1)}
\end{aligned}
$$




\section{B.3 Posteriori marginal de $\lambda_{b}$}

Para os parâmetros $\lambda_{b}$ e $\lambda_{r}$, partindo de B.3, temos:

$$
f\left(\lambda_{b}, \lambda_{r} \mid x, y, z, m\right) \propto
$$

$$
\begin{array}{r}
\propto \int_{0}^{1} \sum_{i=0}^{x} \sum_{j=0}^{y} \sum_{l=0}^{m} \sum_{k=0}^{z} \sum_{r=0}^{k} \sum_{s=0}^{z-k}\left(\begin{array}{c}
x \\
i
\end{array}\right)\left(\begin{array}{c}
y \\
j
\end{array}\right)\left(\begin{array}{c}
m \\
l
\end{array}\right)\left(\begin{array}{c}
z \\
k
\end{array}\right)\left(\begin{array}{c}
k \\
r
\end{array}\right)\left(\begin{array}{c}
z-k \\
s
\end{array}\right)(-1)^{x+y+z-i-j-r-s} \\
p^{a+x+l+k-1}(1-p)^{b+y+z+m-l-k-1} e^{-\lambda_{r} t\left(\frac{\tau}{t}+x-i+k-r+l\right)} e^{-\lambda_{b} t\left(\frac{(\theta-\tau)}{t}+y-j+z-k-s+m-l\right)} \mathbf{d} p \\
\mathbb{1}_{\left(\lambda_{r}>\lambda_{b}>0\right)} \propto
\end{array}
$$

$$
\begin{array}{r}
\propto \sum_{i=0}^{x} \sum_{j=0}^{y} \sum_{l=0}^{m} \sum_{k=0}^{z} \sum_{r=0}^{k} \sum_{s=0}^{z-k}\left(\begin{array}{l}
x \\
i
\end{array}\right)\left(\begin{array}{c}
y \\
j
\end{array}\right)\left(\begin{array}{c}
m \\
l
\end{array}\right)\left(\begin{array}{l}
z \\
k
\end{array}\right)\left(\begin{array}{l}
k \\
r
\end{array}\right)\left(\begin{array}{c}
z-k \\
s
\end{array}\right)(-1)^{x+y+z-i-j-r-s} \\
e^{-\lambda_{r} t\left(\frac{\tau}{t}+x-i+k-r+l\right)} e^{-\lambda_{b} t\left(\frac{(\theta-\tau)}{t}+y-j+z-k-s+m-l\right)} \\
\propto \sum_{i=0}^{1} \sum_{j=0}^{a+x+l+k-1}(1-p)^{b+y+z+m-l-k-1} \mathbf{d} p \mathbb{1}_{\left(\lambda_{r}>\lambda_{b}>0\right)} \propto \\
\frac{\Gamma(a+x+k+k}{k} \sum_{r=0}^{z} \sum_{s=0}^{z-k}\left(\begin{array}{c}
x \\
i
\end{array}\right)\left(\begin{array}{c}
y \\
j
\end{array}\right)\left(\begin{array}{c}
m \\
l
\end{array}\right)\left(\begin{array}{l}
z \\
k
\end{array}\right)\left(\begin{array}{c}
k \\
r
\end{array}\right)\left(\begin{array}{c}
z-k \\
s
\end{array}\right)(-1)^{x+y+z-i-j-r-s} \\
e^{-\lambda_{r} t\left(\frac{\tau}{t}+x-i+k-r+l\right)} e^{-\lambda_{b} t\left(\frac{(\theta-\tau)}{t}+y-j+z-k-s+m-l\right)} \\
\Gamma(a+x+b+y+z+m)
\end{array}
$$

A expressão B.5 representa a densidade a posteriori conjunta de $\lambda_{b}$ e $\lambda_{r}$. Por fim, vamos calcular as marginais, começando por $\lambda_{b}$. 


$$
\propto \int_{\lambda_{b}}^{\infty} \sum_{i=0}^{x} \sum_{j=0}^{y} \sum_{l=0}^{m} \sum_{k=0}^{z} \sum_{r=0}^{k} \sum_{s=0}^{z-k}\left(\begin{array}{l}
x \\
i
\end{array}\right)\left(\begin{array}{l}
y \\
j
\end{array}\right)\left(\begin{array}{l}
m \\
l
\end{array}\right)\left(\begin{array}{l}
z \\
k
\end{array}\right)\left(\begin{array}{l}
k \\
r
\end{array}\right)\left(\begin{array}{c}
z-k \\
s
\end{array}\right)(-1)^{x+y+z-i-j-r-s}
$$

$\frac{\Gamma(a+x+k+l) \Gamma(z+m+b+y-l-k)}{\Gamma(a+x+b+y+z+m)} e^{-\lambda_{r} t\left(\frac{\tau}{t}+x-i+k-r+l\right)} e^{-\lambda_{b} t\left(\frac{(\theta-\tau)}{t}+y-j+z-k-s+m-l\right)} \mathbf{d} \lambda_{r} \mathbb{1}_{\left(0<\lambda_{b}\right)} \propto$

$$
\begin{aligned}
& \propto \sum_{i=0}^{x} \sum_{j=0}^{y} \sum_{l=0}^{m} \sum_{k=0}^{z} \sum_{r=0}^{k} \sum_{s=0}^{z-k}\left(\begin{array}{l}
x \\
i
\end{array}\right)\left(\begin{array}{l}
y \\
j
\end{array}\right)\left(\begin{array}{c}
m \\
l
\end{array}\right)\left(\begin{array}{l}
z \\
k
\end{array}\right)\left(\begin{array}{l}
k \\
r
\end{array}\right)\left(\begin{array}{c}
z-k \\
s
\end{array}\right)(-1)^{x+y+z-i-j-r-s} \\
& \frac{\Gamma(a+x+k+l) \Gamma(z+m+b+y-l-k)}{\Gamma(a+x+b+y+z+m)} e^{-\lambda_{b} t\left(\frac{(\theta-\tau)}{t}+y-j+z-k-s+m-l\right)} \\
& \int_{\lambda_{b}}^{\infty} e^{-\lambda_{r} t\left(\frac{\tau}{t}+x-i+k-r+l\right)} \mathbf{d} \lambda_{r} \mathbb{1}_{\left(0<\lambda_{b}\right)} \propto \\
& \propto \sum_{i=0}^{x} \sum_{j=0}^{y} \sum_{l=0}^{m} \sum_{k=0}^{z} \sum_{r=0}^{k} \sum_{s=0}^{z-k}\left(\begin{array}{l}
x \\
i
\end{array}\right)\left(\begin{array}{l}
y \\
j
\end{array}\right)\left(\begin{array}{l}
m \\
l
\end{array}\right)\left(\begin{array}{l}
z \\
k
\end{array}\right)\left(\begin{array}{l}
k \\
r
\end{array}\right)\left(\begin{array}{c}
z-k \\
s
\end{array}\right)(-1)^{x+y+z-i-j-r-s} \\
& \frac{\Gamma(a+x+k+l) \Gamma(z+m+b+y-l-k)}{\Gamma(a+x+b+y+z+m)} e^{-\lambda_{b} t\left(\frac{(\theta-\tau)}{t}+y-j+z-k-s+m-l\right)} \\
& \frac{-1}{\tau+t(x-i+k-r+l)}\left(0-e^{-\lambda_{b} t\left(\frac{\tau}{t}+x-i+k-r+l\right)}\right) \propto \\
& \propto \sum_{i=0}^{x} \sum_{j=0}^{y} \sum_{l=0}^{m} \sum_{k=0}^{z} \sum_{r=0}^{k} \sum_{s=0}^{z-k}\left(\begin{array}{l}
x \\
i
\end{array}\right)\left(\begin{array}{l}
y \\
j
\end{array}\right)\left(\begin{array}{l}
m \\
l
\end{array}\right)\left(\begin{array}{l}
z \\
k
\end{array}\right)\left(\begin{array}{l}
k \\
r
\end{array}\right)\left(\begin{array}{c}
z-k \\
s
\end{array}\right)(-1)^{x+y+z-i-j-r-s} \\
& \frac{\Gamma(a+x+k+l) \Gamma(b+y+z+m-k-l)}{\Gamma(a+x+b+y+z+m)} \frac{e^{-\lambda_{b} t\left(\frac{\theta}{t}+x+y+z+m-i-j-r-s\right)}}{\tau+t(x-i+k-r+l)} \mathbb{1}_{\left(0<\lambda_{b}\right)}
\end{aligned}
$$




\section{B.4 Posteriori marginal de $\lambda_{r}$}

Partindo novamente da expressão B.5, vamos agora encontrar a posteriori de $\lambda_{r}$. Temos:

$$
f\left(\lambda_{r} \mid x, y, z, m\right) \propto
$$

$$
\propto \int_{0}^{\lambda_{r}} \sum_{i=0}^{x} \sum_{j=0}^{y} \sum_{l=0}^{m} \sum_{k=0}^{z} \sum_{r=0}^{k} \sum_{s=0}^{z-k}\left(\begin{array}{l}
x \\
i
\end{array}\right)\left(\begin{array}{l}
y \\
j
\end{array}\right)\left(\begin{array}{c}
m \\
l
\end{array}\right)\left(\begin{array}{l}
z \\
k
\end{array}\right)\left(\begin{array}{l}
k \\
r
\end{array}\right)\left(\begin{array}{c}
z-k \\
s
\end{array}\right)(-1)^{x+y+z-i-j-r-s}
$$

$\frac{\Gamma(a+x+k+l) \Gamma(z+m+b+y-l-k)}{\Gamma(a+x+b+y+z+m)} e^{-\lambda_{r} t\left(\frac{\tau}{t}+x-i+k-r+l\right)} e^{-\lambda_{b} t\left(\frac{(\theta-\tau)}{t}+y-j+z-k-s+m-l\right)} \mathbf{d} \lambda_{b} \mathbb{1}_{\left(0<\lambda_{r}\right)} \propto$

$$
\begin{array}{r}
\propto \sum_{i=0}^{x} \sum_{j=0}^{y} \sum_{l=0}^{m} \sum_{k=0}^{z} \sum_{r=0}^{k} \sum_{s=0}^{z-k}\left(\begin{array}{l}
x \\
i
\end{array}\right)\left(\begin{array}{c}
y \\
j
\end{array}\right)\left(\begin{array}{c}
m \\
l
\end{array}\right)\left(\begin{array}{l}
z \\
k
\end{array}\right)\left(\begin{array}{l}
k \\
r
\end{array}\right)\left(\begin{array}{c}
z-k \\
s
\end{array}\right)(-1)^{x+y+z-i-j-r-s} \\
\frac{\Gamma(a+x+k+l) \Gamma(z+m+b+y-l-k)}{\Gamma(a+x+b+y+z+m)} e^{-\lambda_{r} t\left(\frac{\tau}{t}+x-i+k-r+l\right)} \\
\int_{0}^{\lambda_{r}} e^{-\lambda_{b} t\left(\frac{(\theta-\tau)}{t}+y-j+z-k-s+m-l\right)} \mathbf{d} \lambda_{b} \mathbb{1}_{\left(0<\lambda_{r}\right)} \propto
\end{array}
$$$$
\propto \sum_{i=0}^{x} \sum_{j=0}^{y} \sum_{l=0}^{m} \sum_{k=0}^{z} \sum_{r=0}^{k} \sum_{s=0}^{z-k}\left(\begin{array}{l}
x \\
i
\end{array}\right)\left(\begin{array}{c}
y \\
j
\end{array}\right)\left(\begin{array}{c}
m \\
l
\end{array}\right)\left(\begin{array}{l}
z \\
k
\end{array}\right)\left(\begin{array}{c}
k \\
r
\end{array}\right)\left(\begin{array}{c}
z-k \\
s
\end{array}\right)(-1)^{x+y+z-i-j-r-s}
$$$$
\frac{\Gamma(a+x+k+l) \Gamma(z+m+b+y-l-k)}{\Gamma(a+x+b+y+z+m)} e^{-\lambda_{r} t\left(\frac{\tau}{t}+x-i+k-r+l\right)}
$$$$
\frac{-1}{\theta-\tau+t(y-j+z-k-s+m-l)}\left[e^{-\lambda_{r} t\left(\frac{\theta-\tau}{t}+y-j+z-k-s+m-l\right)}-1\right] \mathbb{1}_{\left(0<\lambda_{r}\right)} \propto
$$

$$
\propto \sum_{i=0}^{x} \sum_{j=0}^{y} \sum_{l=0}^{m} \sum_{k=0}^{z} \sum_{r=0}^{k} \sum_{s=0}^{z-k}\left(\begin{array}{l}
x \\
i
\end{array}\right)\left(\begin{array}{l}
y \\
j
\end{array}\right)\left(\begin{array}{l}
m \\
l
\end{array}\right)\left(\begin{array}{l}
z \\
k
\end{array}\right)\left(\begin{array}{l}
k \\
r
\end{array}\right)\left(\begin{array}{c}
z-k \\
s
\end{array}\right)(-1)^{x+y+z-i-j-r-s}
$$

$\frac{\Gamma(a+x+k+l) \Gamma(b+y+z+m-k-l)}{\Gamma(a+x+b+y+z+m)} \frac{e^{-\lambda_{r} t\left(\frac{\tau}{t}+x-i+k-r+l\right)}\left[1-e^{-\lambda_{r} t\left(\frac{\theta-\tau}{t}+y-j+z-k-s+m-l\right)}\right]}{\theta-\tau+t(y-j+z-k-s+m-l)} \mathbb{1}_{\left(0<\lambda_{r}\right)}$

A expressão acima é válida caso $\theta-\tau+t(y-j+z-k-s+m-l)$ seja diferente de 0 . Para garantir que isto ocorra, uma possibilidade é estabelecer $\theta>\tau$ nas condições iniciais do experimento, dentre outras. 


\section{Apêndice $\mathrm{C}$}

\section{Desenvolvimento da Expressão 5.5}

Temos que

$$
\begin{aligned}
& f\left(\alpha, p_{1}, \ldots, p_{k-1}, \lambda_{1}, \ldots, \lambda_{k} \mid x_{1}, \ldots, x_{k}, z, m\right) \propto \\
& \propto f\left(p_{1}, \ldots, p_{k-1}, \lambda_{1}, \ldots, \lambda_{k}\right) L\left(\alpha, p_{1}, \ldots, p_{k-1}, \lambda_{1}, \ldots, \lambda_{k} \mid x_{1}, \ldots, x_{k}, z, m\right) \mathbb{1}_{(0<\alpha<1)} \propto \\
& \propto p_{1}^{\alpha_{1}-1} \ldots p_{k}^{\alpha_{k}-1} e^{-\theta_{1} \lambda_{1}} e^{-\theta_{2}\left(\lambda_{2}-\lambda_{1}\right)} \ldots e^{-\theta_{k}\left(\lambda_{k}-\lambda_{k-1}\right)} \\
& {\left[p_{1}\left(1-e^{-\lambda_{1} t}\right)(1-\alpha)\right]^{x_{1}}\left[p_{2}\left(1-e^{-\lambda_{2} t}\right)(1-\alpha)\right]^{x_{2}} \ldots\left[p_{k}\left(1-e^{-\lambda_{k} t}\right)(1-\alpha)\right]^{x_{k}}} \\
& {\left[\sum_{i=1}^{k} p_{i}\left(1-e^{-\lambda_{i} t}\right) \alpha\right]^{z}\left[\sum_{i=1}^{k} p_{i} e^{-\lambda_{i} t}\right]^{m}} \\
& \mathbb{1}_{\left(0 \leq p_{i} \leq 1, \forall i \in\{1, \ldots, k-1\}\right)} \mathbb{1}_{\left(\sum_{i=1}^{k-1} p_{i} \leq 1\right)} \mathbb{1}_{\left(0<\lambda_{1}<\ldots<\lambda_{k}\right)} \mathbb{1}_{(0<\alpha<1)} \propto \\
& \propto p_{1}^{\alpha_{1}-1} \ldots p_{k}^{\alpha_{k}-1} e^{-\theta_{1} \lambda_{1}} e^{-\theta_{2}\left(\lambda_{2}-\lambda_{1}\right)} \ldots e^{-\theta_{k}\left(\lambda_{k}-\lambda_{k-1}\right)} \\
& p_{1}^{x_{1}}\left[\sum_{i_{1}=0}^{x_{1}}\left(\begin{array}{c}
x_{1} \\
i_{1}
\end{array}\right)(1)^{i_{1}}\left(-e^{-\lambda_{1} t}\right)^{x_{1}-i_{1}}\right](1-\alpha)^{x_{1}} \ldots p_{k}^{x_{k}}\left[\sum_{i_{k}=0}^{x_{k}}\left(\begin{array}{c}
x_{k} \\
i_{k}
\end{array}\right)(1)^{i_{k}}\left(-e^{-\lambda_{k} t}\right)^{x_{k}-i_{k}}\right](1-\alpha)^{x_{k}} \\
& \alpha^{z}\left\{\sum_{|\beta|=z} \sum_{j_{1}=0}^{\beta_{1}} \ldots \sum_{j_{k}=0}^{\beta_{k}}\left(\begin{array}{c}
z \\
|\beta|
\end{array}\right)\left(\begin{array}{c}
\beta_{1} \\
j_{1}
\end{array}\right) \ldots\left(\begin{array}{c}
\beta_{k} \\
j_{k}
\end{array}\right) p_{1}^{\beta_{1}} \ldots p_{k}^{\beta_{k}}(-1)^{\sum_{i=1}^{k} \beta_{i}-j_{i}}\left(e^{-\lambda_{1} t}\right)^{\beta_{1}-j_{1}} \ldots\left(e^{-\lambda_{k} t}\right)^{\beta_{k}-j_{k}}\right\} \\
& \left\{\sum_{|\gamma|=m}\left(\begin{array}{c}
m \\
|\gamma|
\end{array}\right) p_{1}^{\gamma_{1}} \ldots p_{k}^{\gamma_{k}}\left(e^{-\lambda_{1} t}\right)^{\gamma_{1}} \ldots\left(e^{-\lambda_{k} t}\right)^{\gamma_{k}}\right\} \mathbb{1}_{\left(0 \leq p_{i} \leq 1, \forall i \in\{1, \ldots, k-1\}\right)} \mathbb{1}_{\left(\sum_{i=1}^{k-1} p_{i} \leq 1\right)} \mathbb{1}_{\left(0<\lambda_{1}<\ldots<\lambda_{k}\right)} \mathbb{1}_{(0<\alpha<1)} \propto \\
& \propto \alpha^{z}(1-\alpha)^{\sum_{i=1}^{k} x_{i}} \sum_{i_{1}=0}^{x_{1}} \ldots \sum_{i_{k}=0}^{x_{k}} \sum_{|\beta|=z} \sum_{j_{1}=0}^{\beta_{1}} \ldots \sum_{j_{k}=0}^{\beta_{k}} \sum_{|\gamma|=m}\left(\begin{array}{c}
x_{1} \\
i_{1}
\end{array}\right) \ldots\left(\begin{array}{c}
x_{k} \\
i_{k}
\end{array}\right)\left(\begin{array}{c}
z \\
|\beta|
\end{array}\right)\left(\begin{array}{c}
\beta_{1} \\
j_{1}
\end{array}\right) \ldots\left(\begin{array}{c}
\beta_{k} \\
j_{k}
\end{array}\right)\left(\begin{array}{c}
m \\
|\gamma|
\end{array}\right) \\
& p_{1}^{\left(\alpha_{1}+x_{1}+\beta_{1}+\gamma_{1}-1\right)} \ldots p_{k}^{\left(\alpha_{k}+x_{k}+\beta_{k}+\gamma_{k}-1\right)}(-1)^{\sum_{l=1}^{k}\left(x_{l}+\beta_{l}-i_{l}-j_{l}\right)} \\
& e^{-\lambda_{1} t\left(\frac{\theta_{1}-\theta_{2}}{t}+x_{1}-i_{1}+\beta_{1}-j_{1}+\gamma_{1}\right)} \ldots e^{-\lambda_{k-1} t\left(\frac{\theta_{k-1}-\theta_{k}}{t}+x_{k-1}-i_{k-1}+\beta_{k-1}-j_{k-1}+\gamma_{k-1}\right)} e^{-\lambda_{k} t\left(\frac{\theta_{k}}{t}+x_{k}-i_{k}+\beta_{k}-j_{k}+\gamma_{k}\right)} \\
& \mathbb{1}_{\left(0 \leq p_{i} \leq 1, \forall i \in\{1, \ldots, k-1\}\right)} \mathbb{1}_{\left(\sum_{i=1}^{k-1} p_{i} \leq 1\right)} \mathbb{1}_{\left(0<\lambda_{1}<\ldots<\lambda_{k}\right)} \mathbb{1}_{(0<\alpha<1)}
\end{aligned}
$$


Na expressão C.1, temos que $|\gamma|=m$ equivale a $\left\{\left(\gamma_{1}, \ldots, \gamma_{k}\right) \in \mathbb{N}^{k}: \sum_{i=1}^{k} \gamma_{i}=m\right\}$. Nesta mesma expressão, temos que $\left(\begin{array}{c}m \\ |\gamma|\end{array}\right)=\frac{m !}{\gamma_{1} ! \ldots \gamma_{k} !}$. 


\section{Apêndice D}

\section{Posterioris marginais de $\alpha, p_{1}, p_{2}, p_{3}, \lambda_{1}, \lambda_{2}$ e $\lambda_{3}$ para 3 tipos de peças}

\section{D.1 Posteriori marginal de $\alpha$}

O resultado é obtido a partir da expressão 5.5, na qual todos os termos dentro das somatórias não dependem de $\alpha$ e, portanto, temos:

$$
f\left(\alpha \mid x_{1}, x_{2}, x_{3}, z, m\right) \propto \alpha^{z}(1-\alpha)^{\left(x_{1}+x_{2}+x_{3}\right)} \mathbb{1}_{(0 \leq \alpha \leq 1)}
$$

Nota-se que $\alpha$ segue, portanto, uma distribuição Beta de parâmetros $z+1$ e $x_{1}+x_{2}+x_{3}+1$. 


\section{D.2 Posteriori marginal de $p_{1}$}

Primeiro, encontramos a marginal conjunta de $\left(p_{1}, p_{2}\right)$. Consideramos que $p_{3}=1-p_{1}-p_{2}$. Assim, para $0 \leq p_{1} \leq 1,0 \leq p_{2} \leq 1$ e $p_{1}+p_{2} \leq 1$, temos:

$\propto \int_{0}^{\infty} \int_{\lambda_{1}}^{\infty} \int_{\lambda_{2}}^{\infty} \sum_{i_{1}=0}^{x_{1}} \sum_{i_{2}=0}^{x_{2}} \sum_{i_{3}=0}^{x_{3}} \sum_{|\beta|=z} \sum_{j_{1}=0}^{\beta_{1}} \sum_{j_{2}=0}^{\beta_{2}} \sum_{j_{3}=0}^{\beta_{3}} \sum_{|\gamma|=m}\left(\begin{array}{c}x_{1} \\ i_{1}\end{array}\right)\left(\begin{array}{c}x_{2} \\ i_{2}\end{array}\right)\left(\begin{array}{c}x_{3} \\ i_{3}\end{array}\right)\left(\begin{array}{c}z \\ |\beta|\end{array}\right)\left(\begin{array}{c}\beta_{1} \\ j_{1}\end{array}\right)\left(\begin{array}{c}\beta_{2} \\ j_{2}\end{array}\right)\left(\begin{array}{c}\beta_{3} \\ j_{3}\end{array}\right)\left(\begin{array}{c}m \\ |\gamma|\end{array}\right)$
$p_{1}^{\left(a_{1}+x_{1}+\beta_{1}+\gamma_{1}-1\right)} p_{2}^{\left(a_{2}+x_{2}+\beta_{2}+\gamma_{2}-1\right)} p_{3}^{\left(a_{3}+x_{3}+\beta_{3}+\gamma_{3}-1\right)}$
$(-1)^{\left(x_{1}-i_{1}+\beta_{1}-j_{1}+x_{2}-i_{2}+\beta_{2}-j_{2}+x_{3}-i_{3}+\beta_{3}-j_{3}\right)}$
$e^{-\lambda_{1} t\left(\frac{\left(\theta_{1}-\theta_{2}\right)}{t}+x_{1}-i_{1}+\beta_{1}-j_{1}+\gamma_{1}\right)} e^{-\lambda_{2} t\left(\frac{\left(\theta_{2}-\theta_{3}\right)}{t}+x_{2}-i_{2}+\beta_{2}-j_{2}+\gamma_{2}\right)} e^{-\lambda_{3} t\left(\frac{\theta_{3}}{t}+x_{3}-i_{3}+\beta_{3}-j_{3}+\gamma_{3}\right)} \mathrm{d} \lambda_{3} \mathrm{~d} \lambda_{2} \mathrm{~d} \lambda_{1} \propto$ $\propto \int_{0}^{\infty} \int_{\lambda_{1}}^{\infty} \sum_{i_{1}=0}^{x_{1}} \sum_{i_{2}=0}^{x_{2}} \sum_{i_{3}=0}^{x_{3}} \sum_{|\beta|=z} \sum_{j_{1}=0}^{\beta_{1}} \sum_{j_{2}=0}^{\beta_{2}} \sum_{j_{3}=0}^{\beta_{3}} \sum_{|\gamma|=m}\left(\begin{array}{c}x_{1} \\ i_{1}\end{array}\right)\left(\begin{array}{c}x_{2} \\ i_{2}\end{array}\right)\left(\begin{array}{c}x_{3} \\ i_{3}\end{array}\right)\left(\begin{array}{c}z \\ |\beta|\end{array}\right)\left(\begin{array}{c}\beta_{1} \\ j_{1}\end{array}\right)\left(\begin{array}{c}\beta_{2} \\ j_{2}\end{array}\right)\left(\begin{array}{c}\beta_{3} \\ j_{3}\end{array}\right)\left(\begin{array}{c}m \\ |\gamma|\end{array}\right)$
$p_{1}^{\left(a_{1}+x_{1}+\beta_{1}+\gamma_{1}-1\right)} p_{2}^{\left(a_{2}+x_{2}+\beta_{2}+\gamma_{2}-1\right)} p_{3}^{\left(a_{3}+x_{3}+\beta_{3}+\gamma_{3}-1\right)}$
$\frac{e^{-\lambda_{1} t\left(\frac{\left(\theta_{1}-\theta_{2}\right)}{t}+x_{1}-i_{1}+\beta_{1}-j_{1}+\gamma_{1}\right)}}{t\left(\frac{\theta_{3}}{t}+x_{3}-i_{3}+\beta_{3}-j_{3}+i_{1}+\beta_{1}-j_{1}+x_{2}-i_{2}+\beta_{2}-j_{2}+x_{3}-i_{3}+\beta_{3}-j_{3}\right)}$
$e^{-\lambda_{2} t\left(\frac{\theta_{2}}{t}+x_{2}-i_{2}+\beta_{2}-j_{2}+\gamma_{2}+x_{3}-i_{3}+\beta_{3}-j_{3}+\gamma_{3}\right)} \mathrm{d} \lambda_{2} \mathrm{~d} \lambda_{1} \propto$ $\propto \int_{0}^{\infty} \sum_{i_{1}=0}^{x_{1}} \sum_{i_{2}=0}^{x_{2}} \sum_{i_{3}=0}^{x_{3}} \sum_{|\beta|=z} \sum_{j_{1}=0}^{\beta_{1}} \sum_{j_{2}=0}^{\beta_{2}} \sum_{j_{3}=0}^{\beta_{3}} \sum_{|\gamma|=m}\left(\begin{array}{c}x_{1} \\ i_{1}\end{array}\right)\left(\begin{array}{c}x_{2} \\ i_{2}\end{array}\right)\left(\begin{array}{c}x_{3} \\ i_{3}\end{array}\right)\left(\begin{array}{c}z \\ |\beta|\end{array}\right)\left(\begin{array}{c}\beta_{1} \\ j_{1}\end{array}\right)\left(\begin{array}{c}\beta_{2} \\ j_{2}\end{array}\right)\left(\begin{array}{c}\beta_{3} \\ j_{3}\end{array}\right)\left(\begin{array}{c}m \\ |\gamma|\end{array}\right)$ $p_{1}^{\left(a_{1}+x_{1}+\beta_{1}+\gamma_{1}-1\right)} p_{2}^{\left(a_{2}+x_{2}+\beta_{2}+\gamma_{2}-1\right)} p_{3}^{\left(a_{3}+x_{3}+\beta_{3}+\gamma_{3}-1\right)}$ $(-1)^{\left(x_{1}-i_{1}+\beta_{1}-j_{1}+x_{2}-i_{2}+\beta_{2}-j_{2}+x_{3}-i_{3}+\beta_{3}-j_{3}\right)}$ $\frac{e^{-\lambda_{1} t\left(\frac{\theta_{1}}{t}+x_{1}-i_{1}+\beta_{1}-j_{1}+\gamma_{1}+x_{2}-i_{2}+\beta_{2}-j_{2}+\gamma_{2}+x_{3}-i_{3}+\beta_{3}-j_{3}+\gamma_{3}\right)}}{t^{2}\left(\frac{\theta_{3}}{t}+x_{3}-i_{3}+\beta_{3}-j_{3}+\gamma_{3}\right)\left(\frac{\theta_{2}}{t}+x_{2}-i_{2}+\beta_{2}-j_{2}+\gamma_{2}+x_{3}-i_{3}+\beta_{3}-j_{3}+\gamma_{3}\right)} \mathrm{d} \lambda_{1} \propto$

$$
\begin{array}{r}
\propto \sum_{i_{1}=0}^{x_{1}} \sum_{i_{2}=0}^{x_{2}} \sum_{i_{3}=0}^{x_{3}} \sum_{|\beta|=z} \sum_{j_{1}=0}^{\beta_{1}} \sum_{j_{2}=0}^{\beta_{2}} \sum_{j_{3}=0}^{\beta_{3}} \sum_{|\gamma|=m}\left(\begin{array}{c}
x_{1} \\
i_{1}
\end{array}\right)\left(\begin{array}{c}
x_{2} \\
i_{2}
\end{array}\right)\left(\begin{array}{c}
x_{3} \\
i_{3}
\end{array}\right)\left(\begin{array}{c}
z \\
|\beta|
\end{array}\right)\left(\begin{array}{c}
\beta_{1} \\
j_{1}
\end{array}\right)\left(\begin{array}{c}
\beta_{2} \\
j_{2}
\end{array}\right)\left(\begin{array}{c}
\beta_{3} \\
j_{3}
\end{array}\right)\left(\begin{array}{c}
m \\
|\gamma|
\end{array}\right) \\
p_{1}^{\left(a_{1}+x_{1}+\beta_{1}+\gamma_{1}-1\right)} p_{2}^{\left(a_{2}+x_{2}+\beta_{2}+\gamma_{2}-1\right)} p_{3}^{\left(a_{3}+x_{3}+\beta_{3}+\gamma_{3}-1\right)} \\
\frac{1}{t^{3}\left(\frac{\theta_{3}}{t}+x_{3}-i_{3}+\beta_{3}-j_{3}+\gamma_{1}+\beta_{1}-j_{1}+x_{2}-i_{2}+\beta_{2}-j_{2}+x_{3}-i_{3}+\beta_{3}-j_{3}\right)}\left(\frac{\theta_{2}}{t}+x_{2}-i_{2}+\beta_{2}-j_{2}+\gamma_{2}+x_{3}-i_{3}+\beta_{3}-j_{3}+\gamma_{3}\right) \\
\frac{1}{\left(\frac{\theta_{1}}{t}+x_{1}-i_{1}+\beta_{1}-j_{1}+\gamma_{1}+x_{2}-i_{2}+\beta_{2}-j_{2}+\gamma_{2}+x_{3}-i_{3}+\beta_{3}-j_{3}+\gamma_{3}\right)}
\end{array}
$$


Agora, podemos calcular a marginal de $p_{1}$ :

$$
\begin{array}{r}
f\left(p_{1} \mid x_{1}, x_{2}, x_{3}, z, m\right) \propto \\
\propto \sum_{i_{1}=0}^{x_{1}} \sum_{i_{2}=0}^{x_{2}} \sum_{i_{3}=0}^{x_{3}} \sum_{|\beta|=z} \sum_{j_{1}=0}^{\beta_{1}} \sum_{j_{2}=0}^{\beta_{2}} \sum_{j_{3}=0}^{\beta_{3}} \sum_{|\gamma|=m}\left(\begin{array}{c}
x_{1} \\
i_{1}
\end{array}\right)\left(\begin{array}{c}
x_{2} \\
i_{2}
\end{array}\right)\left(\begin{array}{c}
x_{3} \\
i_{3}
\end{array}\right)\left(\begin{array}{c}
z \\
|\beta|
\end{array}\right)\left(\begin{array}{c}
\beta_{1} \\
j_{1}
\end{array}\right)\left(\begin{array}{c}
\beta_{2} \\
j_{2}
\end{array}\right)\left(\begin{array}{c}
\beta_{3} \\
j_{3}
\end{array}\right)\left(\begin{array}{c}
m \\
|\gamma|
\end{array}\right) \\
p_{1}^{\left(a_{1}+x_{1}+\beta_{1}+\gamma_{1}-1\right)}\left(1-p_{1}\right)^{\left(a_{2}+x_{2}+\beta_{2}+\gamma_{2}+a_{3}+x_{3}+\beta_{3}+\gamma_{3}-1\right)} \\
\frac{\Gamma\left(a_{2}+x_{2}+\beta_{2}+\gamma_{2}\right) \Gamma\left(a_{3}+x_{3}+\beta_{3}+\gamma_{3}\right)}{\Gamma\left(a_{2}+x_{2}+\beta_{2}+\gamma_{2}+a_{3}+x_{3}+\beta_{3}+\gamma_{3}\right)} \\
\frac{(-1)^{\left(x_{1}-i_{1}+\beta_{1}-j_{1}+x_{2}-i_{2}+\beta_{2}-j_{2}+x_{3}-i_{3}+\beta_{3}-j_{3}\right)}}{t^{3}\left(\frac{\theta_{3}}{t}+x_{3}-i_{3}+\beta_{3}-j_{3}+\gamma_{3}\right)\left(\frac{\theta_{2}}{t}+x_{2}-i_{2}+\beta_{2}-j_{2}+\gamma_{2}+x_{3}-i_{3}+\beta_{3}-j_{3}+\gamma_{3}\right)} \\
\frac{1}{\left(\frac{\theta_{1}}{t}+x_{1}-i_{1}+\beta_{1}-j_{1}+\gamma_{1}+x_{2}-i_{2}+\beta_{2}-j_{2}+\gamma_{2}+x_{3}-i_{3}+\beta_{3}-j_{3}+\gamma_{3}\right)} \\
\mathbb{1}_{\left(0 \leq p_{1} \leq 1\right)}
\end{array}
$$




\section{D.3 Posteriori marginal de $p_{2}$}

De maneira similar a D.3, temos:

$$
f\left(p_{2} \mid x_{1}, x_{2}, x_{3}, z, m\right) \propto
$$

$$
\begin{array}{r}
\propto \sum_{i_{1}=0}^{x_{1}} \sum_{i_{2}=0}^{x_{2}} \sum_{i_{3}=0}^{x_{3}} \sum_{|\beta|=z} \sum_{j_{1}=0}^{\beta_{1}} \sum_{j_{2}=0}^{\beta_{2}} \sum_{j_{3}=0}^{\beta_{3}} \sum_{|\gamma|=m}\left(\begin{array}{c}
x_{1} \\
i_{1}
\end{array}\right)\left(\begin{array}{c}
x_{2} \\
i_{2}
\end{array}\right)\left(\begin{array}{c}
x_{3} \\
i_{3}
\end{array}\right)\left(\begin{array}{c}
z \\
|\beta|
\end{array}\right)\left(\begin{array}{c}
\beta_{1} \\
j_{1}
\end{array}\right)\left(\begin{array}{c}
\beta_{2} \\
j_{2}
\end{array}\right)\left(\begin{array}{c}
\beta_{3} \\
j_{3}
\end{array}\right)\left(\begin{array}{c}
m \\
|\gamma|
\end{array}\right) \\
p_{2}^{\left(a_{2}+x_{2}+\beta_{2}+\gamma_{2}-1\right)}\left(1-p_{2}\right)^{\left(a_{1}+x_{1}+\beta_{1}+\gamma_{1}+a_{3}+x_{3}+\beta_{3}+\gamma_{3}-1\right)} \\
\frac{\Gamma\left(a_{1}+x_{1}+\beta_{1}+\gamma_{1}\right) \Gamma\left(a_{3}+x_{3}+\beta_{3}+\gamma_{3}\right)}{\Gamma\left(a_{1}+x_{1}+\beta_{1}+\gamma_{1}+a_{3}+x_{3}+\beta_{3}+\gamma_{3}\right)} \\
\frac{1-1)^{\left(x_{1}-i_{1}+\beta_{1}-j_{1}+x_{2}-i_{2}+\beta_{2}-j_{2}+x_{3}-i_{3}+\beta_{3}-j_{3}\right)}}{t^{3}\left(\frac{\theta_{3}}{t}+x_{3}-i_{3}+\beta_{3}-j_{3}+\gamma_{3}\right)\left(\frac{\theta_{2}}{t}+x_{2}-i_{2}+\beta_{2}-j_{2}+\gamma_{2}+x_{3}-i_{3}+\beta_{3}-j_{3}+\gamma_{3}\right)} \\
\frac{1}{\left(\frac{\theta_{1}}{t}+x_{1}-i_{1}+\beta_{1}-j_{1}+\gamma_{1}+x_{2}-i_{2}+\beta_{2}-j_{2}+\gamma_{2}+x_{3}-i_{3}+\beta_{3}-j_{3}+\gamma_{3}\right)} \\
\mathbb{1}_{\left(0 \leq p_{2} \leq 1\right)}
\end{array}
$$




\section{D.4 Posteriori marginal de $p_{3}$}

Iremos também calcular a posteriori para $p_{3}=1-p_{1}-p_{2}$ :

$$
f\left(p_{3} \mid x_{1}, x_{2}, x_{3}, z, m\right) \propto
$$

$$
\begin{array}{r}
\propto \sum_{i_{1}=0}^{x_{1}} \sum_{i_{2}=0}^{x_{2}} \sum_{i_{3}=0}^{x_{3}} \sum_{|\beta|=z} \sum_{j_{1}=0}^{\beta_{1}} \sum_{j_{2}=0}^{\beta_{2}} \sum_{j_{3}=0}^{\beta_{3}} \sum_{|\gamma|=m}\left(\begin{array}{c}
x_{1} \\
i_{1}
\end{array}\right)\left(\begin{array}{c}
x_{2} \\
i_{2}
\end{array}\right)\left(\begin{array}{c}
x_{3} \\
i_{3}
\end{array}\right)\left(\begin{array}{c}
z \\
|\beta|
\end{array}\right)\left(\begin{array}{c}
\beta_{1} \\
j_{1}
\end{array}\right)\left(\begin{array}{c}
\beta_{2} \\
j_{2}
\end{array}\right)\left(\begin{array}{c}
\beta_{3} \\
j_{3}
\end{array}\right)\left(\begin{array}{l}
m \\
|\gamma|
\end{array}\right) \\
p_{3}^{\left(a_{3}+x_{3}+\beta_{3}+\gamma_{3}-1\right)}\left(1-p_{3}\right)^{\left(a_{1}+x_{1}+\beta_{1}+\gamma_{1}+a_{2}+x_{2}+\beta_{2}+\gamma_{2}-1\right)} \\
\frac{\Gamma\left(a_{1}+x_{1}+\beta_{1}+\gamma_{1}\right) \Gamma\left(a_{2}+x_{2}+\beta_{2}+\gamma_{2}\right)}{\Gamma\left(a_{1}+x_{1}+\beta_{1}+\gamma_{1}+a_{2}+x_{2}+\beta_{2}+\gamma_{2}\right)} \\
\frac{1-1)^{\left(x_{1}-i_{1}+\beta_{1}-j_{1}+x_{2}-i_{2}+\beta_{2}-j_{2}+x_{3}-i_{3}+\beta_{3}-j_{3}\right)}}{t^{3}\left(\frac{\theta_{3}}{t}+x_{3}-i_{3}+\beta_{3}-j_{3}+\gamma_{3}\right)\left(\frac{\theta_{2}}{t}+x_{2}-i_{2}+\beta_{2}-j_{2}+\gamma_{2}+x_{3}-i_{3}+\beta_{3}-j_{3}+\gamma_{3}\right)} \\
\frac{1}{\left(\frac{\theta_{1}}{t}+x_{1}-i_{1}+\beta_{1}-j_{1}+\gamma_{1}+x_{2}-i_{2}+\beta_{2}-j_{2}+\gamma_{2}+x_{3}-i_{3}+\beta_{3}-j_{3}+\gamma_{3}\right)} \\
\mathbb{1}_{\left(0 \leq p_{3} \leq 1\right)}
\end{array}
$$




\section{D.5 Posteriori marginal de $\lambda_{1}$}

Primeiramente, iremos calcular a marginal conjunta de $\left(\lambda_{1}, \lambda_{2}, \lambda_{3}\right)$. Temos:

$$
f\left(\lambda_{1}, \lambda_{2}, \lambda_{3} \mid x_{1}, x_{2}, x_{3}, z, m\right)=\iint_{p_{1}+p_{2} \leq 1} \int_{0}^{1} f\left(\alpha, p_{1}, p_{2}, \lambda_{1}, \lambda_{2}, \lambda_{3} \mid x_{1}, x_{2}, x_{3}, z, m\right) \mathrm{d} \alpha \mathrm{d} p_{1} \mathrm{~d} p_{2}
$$

Temos que $\alpha$ não influi na posteriori dos $\lambda$ 's. Integrando nas proporções $\left(p_{1}, p_{2}\right)$ temos, do modelo Dirichlet, que:

$$
\begin{gathered}
\iint_{p_{1}+p_{2} \leq 1} p_{1}^{\left(a_{1}+x_{1}+\beta_{1}+\gamma_{1}-1\right)} p_{2}^{\left(a_{2}+x_{2}+\beta_{2}+\gamma_{2}-1\right)}\left(1-p_{1}-p_{2}\right)^{\left(a_{3}+x_{3}+\beta_{3}+\gamma_{3}-1\right)} \\
\frac{\Gamma\left(a_{1}+x_{1}+\beta_{1}+\gamma_{1}+a_{2}+x_{2}+\beta_{2}+\gamma_{2}+a_{3}+x_{3}+\beta_{3}+\gamma_{3}\right)}{\Gamma\left(a_{1}+x_{1}+\beta_{1}+\gamma_{1}\right) \Gamma\left(a_{2}+x_{2}+\beta_{2}+\gamma_{2}\right) \Gamma\left(a_{3}+x_{3}+\beta_{3}+\gamma_{3}\right)} \mathrm{d} p_{1} \mathrm{~d} p_{2}=1
\end{gathered}
$$

Com isso,

$$
f\left(\lambda_{1}, \lambda_{2}, \lambda_{3} \mid x_{1}, x_{2}, x_{3}, z, m\right) \propto
$$

$$
\begin{array}{r}
\propto \sum_{i_{1}=0}^{x_{1}} \sum_{i_{2}=0}^{x_{2}} \sum_{i_{3}=0}^{x_{3}} \sum_{|\beta|=z} \sum_{j_{1}=0}^{\beta_{1}} \sum_{j_{2}=0}^{\beta_{2}} \sum_{j_{3}=0}^{\beta_{3}} \sum_{|\gamma|=m}\left(\begin{array}{c}
x_{1} \\
i_{1}
\end{array}\right)\left(\begin{array}{c}
x_{2} \\
i_{2}
\end{array}\right)\left(\begin{array}{c}
x_{3} \\
i_{3}
\end{array}\right)\left(\begin{array}{c}
z \\
|\beta|
\end{array}\right)\left(\begin{array}{c}
\beta_{1} \\
j_{1}
\end{array}\right)\left(\begin{array}{c}
\beta_{2} \\
j_{2}
\end{array}\right)\left(\begin{array}{c}
\beta_{3} \\
j_{3}
\end{array}\right)\left(\begin{array}{c}
m \\
|\gamma|
\end{array}\right) \\
\frac{\Gamma\left(a_{1}+x_{1}+\beta_{1}+\gamma_{1}\right) \Gamma\left(a_{2}+x_{2}+\beta_{2}+\gamma_{2}\right) \Gamma\left(a_{3}+x_{3}+\beta_{3}+\gamma_{3}\right)}{\Gamma\left(a_{1}+x_{1}+\beta_{1}+\gamma_{1}+a_{2}+x_{2}+\beta_{2}+\gamma_{2}+a_{3}+x_{3}+\beta_{3}+\gamma_{3}\right)} \\
(-1)^{\left(x_{1}-i_{1}+\beta_{1}-j_{1}+x_{2}-i_{2}+\beta_{2}-j_{2}+x_{3}-i_{3}+\beta_{3}-j_{3}\right)} \\
e^{-\lambda_{1} t\left(\frac{\left(\theta_{1}-\theta_{2}\right)}{t}+x_{1}-i_{1}+\beta_{1}-j_{1}+\gamma_{1}\right)} e^{-\lambda_{2} t\left(\frac{\left(\theta_{2}-\theta_{3}\right)}{t}+x_{2}-i_{2}+\beta_{2}-j_{2}+\gamma_{2}\right)} e^{-\lambda_{3} t\left(\frac{\theta_{3}}{t}+x_{3}-i_{3}+\beta_{3}-j_{3}+\gamma_{3}\right)} \\
\mathbb{1}_{\left(0<\lambda_{1}<\lambda_{2}<\lambda_{3}\right)}
\end{array}
$$


Marginalmente, temos, para $\lambda_{1}>0$, que:

$f\left(\lambda_{1} \mid x_{1}, x_{2}, x_{3}, z, m\right) \propto$

$\propto \int_{\lambda_{1}}^{\infty} \int_{\lambda_{2}}^{\infty} \sum_{i_{1}=0}^{x_{1}} \sum_{i_{2}=0}^{x_{2}} \sum_{i_{3}=0}^{x_{3}} \sum_{|\beta|=z} \sum_{j_{1}=0}^{\beta_{1}} \sum_{j_{2}=0}^{\beta_{2}} \sum_{j_{3}=0}^{\beta_{3}} \sum_{|\gamma|=m}\left(\begin{array}{c}x_{1} \\ i_{1}\end{array}\right)\left(\begin{array}{c}x_{2} \\ i_{2}\end{array}\right)\left(\begin{array}{c}x_{3} \\ i_{3}\end{array}\right)\left(\begin{array}{c}z \\ |\beta|\end{array}\right)\left(\begin{array}{c}\beta_{1} \\ j_{1}\end{array}\right)\left(\begin{array}{c}\beta_{2} \\ j_{2}\end{array}\right)\left(\begin{array}{c}\beta_{3} \\ j_{3}\end{array}\right)\left(\begin{array}{c}m \\ |\gamma|\end{array}\right)$
$\frac{\Gamma\left(a_{1}+x_{1}+\beta_{1}+\gamma_{1}\right) \Gamma\left(a_{2}+x_{2}+\beta_{2}+\gamma_{2}\right) \Gamma\left(a_{3}+x_{3}+\beta_{3}+\gamma_{3}\right)}{\Gamma\left(a_{1}+x_{1}+\beta_{1}+\gamma_{1}+a_{2}+x_{2}+\beta_{2}+\gamma_{2}+a_{3}+x_{3}+\beta_{3}+\gamma_{3}\right)}$
$(-1)\left(x_{1}-i_{1}+\beta_{1}-j_{1}+x_{2}-i_{2}+\beta_{2}-j_{2}+x_{3}-i_{3}+\beta_{3}-j_{3}\right)$
$e^{-\lambda_{1} t\left(\frac{\left(\theta_{1}-\theta_{2}\right)}{t}+x_{1}-i_{1}+\beta_{1}-j_{1}+\gamma_{1}\right)} e^{-\lambda_{2} t\left(\frac{\left(\theta_{2}-\theta_{3}\right)}{t}+x_{2}-i_{2}+\beta_{2}-j_{2}+\gamma_{2}\right)} e^{-\lambda_{3} t\left(\frac{\theta_{3}}{t}+x_{3}-i_{3}+\beta_{3}-j_{3}+\gamma_{3}\right)} \mathrm{d} \lambda_{3} \mathrm{~d} \lambda_{2} \propto$

$$
\begin{aligned}
& \propto \int_{\lambda_{1}}^{\infty} \sum_{i_{1}=0}^{x_{1}} \sum_{i_{2}=0}^{x_{2}} \sum_{i_{3}=0}^{x_{3}} \sum_{|\beta|=z} \sum_{j_{1}=0}^{\beta_{1}} \sum_{j_{2}=0}^{\beta_{2}} \sum_{j_{3}=0}^{\beta_{3}} \sum_{|\gamma|=m}\left(\begin{array}{c}
x_{1} \\
i_{1}
\end{array}\right)\left(\begin{array}{c}
x_{2} \\
i_{2}
\end{array}\right)\left(\begin{array}{c}
x_{3} \\
i_{3}
\end{array}\right)\left(\begin{array}{c}
z \\
|\beta|
\end{array}\right)\left(\begin{array}{c}
\beta_{1} \\
j_{1}
\end{array}\right)\left(\begin{array}{c}
\beta_{2} \\
j_{2}
\end{array}\right)\left(\begin{array}{c}
\beta_{3} \\
j_{3}
\end{array}\right)\left(\begin{array}{c}
m \\
|\gamma|
\end{array}\right) \\
& \frac{\Gamma\left(a_{1}+x_{1}+\beta_{1}+\gamma_{1}\right) \Gamma\left(a_{2}+x_{2}+\beta_{2}+\gamma_{2}\right) \Gamma\left(a_{3}+x_{3}+\beta_{3}+\gamma_{3}\right)}{\Gamma\left(a_{1}+x_{1}+\beta_{1}+\gamma_{1}+a_{2}+x_{2}+\beta_{2}+\gamma_{2}+a_{3}+x_{3}+\beta_{3}+\gamma_{3}\right)} \\
& (-1)^{\left(x_{1}-i_{1}+\beta_{1}-j_{1}+x_{2}-i_{2}+\beta_{2}-j_{2}+x_{3}-i_{3}+\beta_{3}-j_{3}\right)} \\
& \frac{e^{-\lambda_{1} t\left(\frac{\left(\theta_{1}-\theta_{2}\right)}{t}+x_{1}-i_{1}+\beta_{1}-j_{1}+\gamma_{1}\right)}}{t\left(\frac{\theta_{3}}{t}+x_{3}-i_{3}+\beta_{3}-j_{3}+\gamma_{3}\right)} e^{-\lambda_{2} t\left(\frac{\theta_{2}}{t}+x_{2}-i_{2}+\beta_{2}-j_{2}+\gamma_{2}+x_{3}-i_{3}+\beta_{3}-j_{3}+\gamma_{3}\right)} \mathrm{d} \lambda_{2} \propto \\
& \propto \sum_{i_{1}=0}^{x_{1}} \sum_{i_{2}=0}^{x_{2}} \sum_{i_{3}=0}^{x_{3}} \sum_{|\beta|=z} \sum_{j_{1}=0}^{\beta_{1}} \sum_{j_{2}=0}^{\beta_{2}} \sum_{j_{3}=0}^{\beta_{3}} \sum_{|\gamma|=m}\left(\begin{array}{c}
x_{1} \\
i_{1}
\end{array}\right)\left(\begin{array}{c}
x_{2} \\
i_{2}
\end{array}\right)\left(\begin{array}{c}
x_{3} \\
i_{3}
\end{array}\right)\left(\begin{array}{c}
z \\
|\beta|
\end{array}\right)\left(\begin{array}{c}
\beta_{1} \\
j_{1}
\end{array}\right)\left(\begin{array}{c}
\beta_{2} \\
j_{2}
\end{array}\right)\left(\begin{array}{c}
\beta_{3} \\
j_{3}
\end{array}\right)\left(\begin{array}{c}
m \\
|\gamma|
\end{array}\right) \\
& \frac{\Gamma\left(a_{1}+x_{1}+\beta_{1}+\gamma_{1}\right) \Gamma\left(a_{2}+x_{2}+\beta_{2}+\gamma_{2}\right) \Gamma\left(a_{3}+x_{3}+\beta_{3}+\gamma_{3}\right)}{\Gamma\left(a_{1}+x_{1}+\beta_{1}+\gamma_{1}+a_{2}+x_{2}+\beta_{2}+\gamma_{2}+a_{3}+x_{3}+\beta_{3}+\gamma_{3}\right)} \\
& (-1)^{\left(x_{1}-i_{1}+\beta_{1}-j_{1}+x_{2}-i_{2}+\beta_{2}-j_{2}+x_{3}-i_{3}+\beta_{3}-j_{3}\right)} \\
& e^{-\lambda_{1} t\left(\frac{\theta_{1}}{t}+x_{1}-i_{1}+\beta_{1}-j_{1}+\gamma_{1}+x_{2}-i_{2}+\beta_{2}-j_{2}+\gamma_{2}+x_{3}-i_{3}+\beta_{3}-j_{3}+\gamma_{3}\right)} \\
& t^{2}\left(\frac{\theta_{3}}{t}+x_{3}-i_{3}+\beta_{3}-j_{3}+\gamma_{3}\right)\left(\frac{\theta_{2}}{t}+x_{2}-i_{2}+\beta_{2}-j_{2}+\gamma_{2}+x_{3}-i_{3}+\beta_{3}-j_{3}+\gamma_{3}\right)
\end{aligned}
$$




\section{D.6 Posteriori marginal de $\lambda_{2}$}

Temos, para $\lambda_{2}>0$, que:

$f\left(\lambda_{2} \mid x_{1}, x_{2}, x_{3}, z, m\right) \propto$

$$
\begin{array}{r}
\propto \int_{0}^{\lambda_{2}} \int_{\lambda_{2}}^{\infty} \sum_{i_{1}=0}^{x_{1}} \sum_{i_{2}=0}^{x_{2}} \sum_{i_{3}=0}^{x_{3}} \sum_{|\beta|=z} \sum_{j_{1}=0}^{\beta_{1}} \sum_{j_{2}=0}^{\beta_{2}} \sum_{j_{3}=0}^{\beta_{3}} \sum_{|\gamma|=m}\left(\begin{array}{c}
x_{1} \\
i_{1}
\end{array}\right)\left(\begin{array}{c}
x_{2} \\
i_{2}
\end{array}\right)\left(\begin{array}{c}
x_{3} \\
i_{3}
\end{array}\right)\left(\begin{array}{c}
z \\
|\beta|
\end{array}\right)\left(\begin{array}{c}
\beta_{1} \\
j_{1}
\end{array}\right)\left(\begin{array}{c}
\beta_{2} \\
j_{2}
\end{array}\right)\left(\begin{array}{c}
\beta_{3} \\
j_{3}
\end{array}\right)\left(\begin{array}{c}
m \\
|\gamma|
\end{array}\right) \\
\frac{\Gamma\left(a_{1}+x_{1}+\beta_{1}+\gamma_{1}\right) \Gamma\left(a_{2}+x_{2}+\beta_{2}+\gamma_{2}\right) \Gamma\left(a_{3}+x_{3}+\beta_{3}+\gamma_{3}\right)}{\Gamma\left(a_{1}+x_{1}+\beta_{1}+\gamma_{1}+a_{2}+x_{2}+\beta_{2}+\gamma_{2}+a_{3}+x_{3}+\beta_{3}+\gamma_{3}\right)} \\
(-1)^{\left(x_{1}-i_{1}+\beta_{1}-j_{1}+x_{2}-i_{2}+\beta_{2}-j_{2}+x_{3}-i_{3}+\beta_{3}-j_{3}\right)} \\
e^{-\lambda_{1} t\left(\frac{\left(\theta_{1}-\theta_{2}\right)}{t}+x_{1}-i_{1}+\beta_{1}-j_{1}+\gamma_{1}\right)} e^{-\lambda_{2} t\left(\frac{\left(\theta_{2}-\theta_{3}\right)}{t}+x_{2}-i_{2}+\beta_{2}-j_{2}+\gamma_{2}\right)} e^{-\lambda_{3} t\left(\frac{\theta_{3}}{t}+x_{3}-i_{3}+\beta_{3}-j_{3}+\gamma_{3}\right)} \mathrm{d} \lambda_{3} \mathrm{~d} \lambda_{1} \propto
\end{array}
$$

$$
\begin{array}{r}
\propto \int_{0}^{\lambda_{2}} \sum_{i_{1}=0}^{x_{1}} \sum_{i_{2}=0}^{x_{2}} \sum_{i_{3}=0}^{x_{3}} \sum_{|\beta|=z} \sum_{j_{1}=0}^{\beta_{1}} \sum_{j_{2}=0}^{\beta_{2}} \sum_{j_{3}=0}^{\beta_{3}} \sum_{|\gamma|=m}\left(\begin{array}{c}
x_{1} \\
i_{1}
\end{array}\right)\left(\begin{array}{c}
x_{2} \\
i_{2}
\end{array}\right)\left(\begin{array}{c}
x_{3} \\
i_{3}
\end{array}\right)\left(\begin{array}{c}
z \\
|\beta|
\end{array}\right)\left(\begin{array}{c}
\beta_{1} \\
j_{1}
\end{array}\right)\left(\begin{array}{c}
\beta_{2} \\
j_{2}
\end{array}\right)\left(\begin{array}{c}
\beta_{3} \\
j_{3}
\end{array}\right)\left(\begin{array}{c}
m \\
|\gamma|
\end{array}\right) \\
\frac{\Gamma\left(a_{1}+x_{1}+\beta_{1}+\gamma_{1}\right) \Gamma\left(a_{2}+x_{2}+\beta_{2}+\gamma_{2}\right) \Gamma\left(a_{3}+x_{3}+\beta_{3}+\gamma_{3}\right)}{\Gamma\left(a_{1}+x_{1}+\beta_{1}+\gamma_{1}+a_{2}+x_{2}+\beta_{2}+\gamma_{2}+a_{3}+x_{3}+\beta_{3}+\gamma_{3}\right)}
\end{array}
$$$$
(-1)^{\left(x_{1}-i_{1}+\beta_{1}-j_{1}+x_{2}-i_{2}+\beta_{2}-j_{2}+x_{3}-i_{3}+\beta_{3}-j_{3}\right)}
$$$$
\frac{e^{-\lambda_{2} t\left(\frac{\theta_{2}}{t}+x_{2}-i_{2}+\beta_{2}-j_{2}+\gamma_{2}+x_{3}-i_{3}+\beta_{3}-j_{3}+\gamma_{3}\right)}}{t\left(\frac{\theta_{3}}{t}+x_{3}-i_{3}+\beta_{3}-j_{3}+\gamma_{3}\right)} e^{-\lambda_{1} t\left(\frac{\left(\theta_{1}-\theta_{2}\right)}{t}+x_{1}-i_{1}+\beta_{1}-j_{1}+\gamma_{1}\right)} \mathrm{d} \lambda_{1} \propto
$$

$$
\begin{array}{r}
\propto \sum_{i_{1}=0}^{x_{1}} \sum_{i_{2}=0}^{x_{2}} \sum_{i_{3}=0}^{x_{3}} \sum_{|\beta|=z} \sum_{j_{1}=0}^{\beta_{1}} \sum_{j_{2}=0}^{\beta_{2}} \sum_{j_{3}=0}^{\beta_{3}} \sum_{|\gamma|=m}\left(\begin{array}{c}
x_{1} \\
i_{1}
\end{array}\right)\left(\begin{array}{c}
x_{2} \\
i_{2}
\end{array}\right)\left(\begin{array}{c}
x_{3} \\
i_{3}
\end{array}\right)\left(\begin{array}{c}
z \\
|\beta|
\end{array}\right)\left(\begin{array}{c}
\beta_{1} \\
j_{1}
\end{array}\right)\left(\begin{array}{c}
\beta_{2} \\
j_{2}
\end{array}\right)\left(\begin{array}{c}
\beta_{3} \\
j_{3}
\end{array}\right)\left(\begin{array}{c}
m \\
|\gamma|
\end{array}\right) \\
\frac{\Gamma\left(a_{1}+x_{1}+\beta_{1}+\gamma_{1}\right) \Gamma\left(a_{2}+x_{2}+\beta_{2}+\gamma_{2}\right) \Gamma\left(a_{3}+x_{3}+\beta_{3}+\gamma_{3}\right)}{\Gamma\left(a_{1}+x_{1}+\beta_{1}+\gamma_{1}+a_{2}+x_{2}+\beta_{2}+\gamma_{2}+a_{3}+x_{3}+\beta_{3}+\gamma_{3}\right)}
\end{array}
$$

$$
(-1)^{\left(x_{1}-i_{1}+\beta_{1}-j_{1}+x_{2}-i_{2}+\beta_{2}-j_{2}+x_{3}-i_{3}+\beta_{3}-j_{3}\right)}
$$

$\frac{e^{-\lambda_{2} t\left(\frac{\theta_{2}}{t}+x_{2}-i_{2}+\beta_{2}-j_{2}+\gamma_{2}+x_{3}-i_{3}+\beta_{3}-j_{3}+\gamma_{3}\right)}-e^{-\lambda_{2} t\left(\frac{\theta_{1}}{t}+x_{1}-i_{1}+\beta_{1}-j_{1}+\gamma_{1}+x_{2}-i_{2}+\beta_{2}-j_{2}+\gamma_{2}+x_{3}-i_{3}+\beta_{3}-j_{3}+\gamma_{3}\right)}}{t^{2}\left(\frac{\theta_{3}}{t}+x_{3}-i_{3}+\beta_{3}-j_{3}+\gamma_{3}\right)\left(\frac{\left(\theta_{1}-\theta_{2}\right)}{t}+x_{1}-i_{1}+\beta_{1}-j_{1}+\gamma_{1}\right)}$

De maneira similar à observada em B.4, para que a expressão acima seja válida é necessário garantir que $\left(\frac{\left(\theta_{1}-\theta_{2}\right)}{t}+x_{1}-i_{1}+\beta_{1}-j_{1}+\gamma_{1}\right)$ seja diferente de 0 . Isto pode ser feito, por exemplo, estabelecendo $\theta_{1}>\theta_{2}$. 


\section{D.7 Posteriori marginal de $\lambda_{3}$}

Por fim temos, para $\lambda_{3}>0$, que:

$f\left(\lambda_{3} \mid x_{1}, x_{2}, x_{3}, z, m\right) \propto$

$$
\begin{aligned}
& \propto \int_{0}^{\lambda_{3}} \int_{0}^{\lambda_{2}} \sum_{i_{1}=0}^{x_{1}} \sum_{i_{2}=0}^{x_{2}} \sum_{i_{3}=0}^{x_{3}} \sum_{|\beta|=z} \sum_{j_{1}=0}^{\beta_{1}} \sum_{j_{2}=0}^{\beta_{2}} \sum_{j_{3}=0}^{\beta_{3}} \sum_{|\gamma|=m}\left(\begin{array}{c}
x_{1} \\
i_{1}
\end{array}\right)\left(\begin{array}{c}
x_{2} \\
i_{2}
\end{array}\right)\left(\begin{array}{c}
x_{3} \\
i_{3}
\end{array}\right)\left(\begin{array}{c}
z \\
|\beta|
\end{array}\right)\left(\begin{array}{c}
\beta_{1} \\
j_{1}
\end{array}\right)\left(\begin{array}{c}
\beta_{2} \\
j_{2}
\end{array}\right)\left(\begin{array}{c}
\beta_{3} \\
j_{3}
\end{array}\right)\left(\begin{array}{c}
m \\
|\gamma|
\end{array}\right) \\
& \frac{\Gamma\left(a_{1}+x_{1}+\beta_{1}+\gamma_{1}\right) \Gamma\left(a_{2}+x_{2}+\beta_{2}+\gamma_{2}\right) \Gamma\left(a_{3}+x_{3}+\beta_{3}+\gamma_{3}\right)}{\Gamma\left(a_{1}+x_{1}+\beta_{1}+\gamma_{1}+a_{2}+x_{2}+\beta_{2}+\gamma_{2}+a_{3}+x_{3}+\beta_{3}+\gamma_{3}\right)} \\
& (-1)^{\left(x_{1}-i_{1}+\beta_{1}-j_{1}+x_{2}-i_{2}+\beta_{2}-j_{2}+x_{3}-i_{3}+\beta_{3}-j_{3}\right)} \\
& e^{-\lambda_{1} t\left(\frac{\left(\theta_{1}-\theta_{2}\right)}{t}+x_{1}-i_{1}+\beta_{1}-j_{1}+\gamma_{1}\right)} e^{-\lambda_{2} t\left(\frac{\left(\theta_{2}-\theta_{3}\right)}{t}+x_{2}-i_{2}+\beta_{2}-j_{2}+\gamma_{2}\right)} e^{-\lambda_{3} t\left(\frac{\theta_{3}}{t}+x_{3}-i_{3}+\beta_{3}-j_{3}+\gamma_{3}\right)} \mathrm{d} \lambda_{1} \mathrm{~d} \lambda_{2} \propto \\
& \propto \int_{0}^{\lambda_{3}} \sum_{i_{1}=0}^{x_{1}} \sum_{i_{2}=0}^{x_{2}} \sum_{i_{3}=0}^{x_{3}} \sum_{|\beta|=z} \sum_{j_{1}=0}^{\beta_{1}} \sum_{j_{2}=0}^{\beta_{2}} \sum_{j_{3}=0}^{\beta_{3}} \sum_{|\gamma|=m}\left(\begin{array}{c}
x_{1} \\
i_{1}
\end{array}\right)\left(\begin{array}{c}
x_{2} \\
i_{2}
\end{array}\right)\left(\begin{array}{c}
x_{3} \\
i_{3}
\end{array}\right)\left(\begin{array}{c}
z \\
|\beta|
\end{array}\right)\left(\begin{array}{c}
\beta_{1} \\
j_{1}
\end{array}\right)\left(\begin{array}{c}
\beta_{2} \\
j_{2}
\end{array}\right)\left(\begin{array}{c}
\beta_{3} \\
j_{3}
\end{array}\right)\left(\begin{array}{c}
m \\
|\gamma|
\end{array}\right) \\
& \frac{\Gamma\left(a_{1}+x_{1}+\beta_{1}+\gamma_{1}\right) \Gamma\left(a_{2}+x_{2}+\beta_{2}+\gamma_{2}\right) \Gamma\left(a_{3}+x_{3}+\beta_{3}+\gamma_{3}\right)}{\Gamma\left(a_{1}+x_{1}+\beta_{1}+\gamma_{1}+a_{2}+x_{2}+\beta_{2}+\gamma_{2}+a_{3}+x_{3}+\beta_{3}+\gamma_{3}\right)} \\
& (-1)^{\left(x_{1}-i_{1}+\beta_{1}-j_{1}+x_{2}-i_{2}+\beta_{2}-j_{2}+x_{3}-i_{3}+\beta_{3}-j_{3}\right)} \\
& \frac{e^{-\lambda_{3} t\left(\frac{\theta_{3}}{t}+x_{3}-i_{3}+\beta_{3}-j_{3}+\gamma_{3}\right)}}{t\left(\frac{\left(\theta_{1}-\theta_{2}\right)}{t}+x_{1}-i_{1}+\beta_{1}-j_{1}+\gamma_{1}\right)} \\
& {\left[e^{-\lambda_{2} t\left(\frac{\left(\theta_{2}-\theta_{3}\right)}{t}+x_{2}-i_{2}+\beta_{2}-j_{2}+\gamma_{2}\right)}-e^{-\lambda_{2} t\left(\frac{\left(\theta_{1}-\theta_{3}\right)}{t}+x_{1}-i_{1}+\beta_{1}-j_{1}+\gamma_{1}+x_{2}-i_{2}+\beta_{2}-j_{2}+\gamma_{2}\right)}\right] \mathrm{d} \lambda_{2} \propto} \\
& \propto \sum_{i_{1}=0}^{x_{1}} \sum_{i_{2}=0}^{x_{2}} \sum_{i_{3}=0}^{x_{3}} \sum_{|\beta|=z} \sum_{j_{1}=0}^{\beta_{1}} \sum_{j_{2}=0}^{\beta_{2}} \sum_{j_{3}=0}^{\beta_{3}} \sum_{|\gamma|=m}\left(\begin{array}{c}
x_{1} \\
i_{1}
\end{array}\right)\left(\begin{array}{c}
x_{2} \\
i_{2}
\end{array}\right)\left(\begin{array}{c}
x_{3} \\
i_{3}
\end{array}\right)\left(\begin{array}{c}
z \\
|\beta|
\end{array}\right)\left(\begin{array}{c}
\beta_{1} \\
j_{1}
\end{array}\right)\left(\begin{array}{c}
\beta_{2} \\
j_{2}
\end{array}\right)\left(\begin{array}{c}
\beta_{3} \\
j_{3}
\end{array}\right)\left(\begin{array}{c}
m \\
|\gamma|
\end{array}\right) \\
& \frac{\Gamma\left(a_{1}+x_{1}+\beta_{1}+\gamma_{1}\right) \Gamma\left(a_{2}+x_{2}+\beta_{2}+\gamma_{2}\right) \Gamma\left(a_{3}+x_{3}+\beta_{3}+\gamma_{3}\right)}{\Gamma\left(a_{1}+x_{1}+\beta_{1}+\gamma_{1}+a_{2}+x_{2}+\beta_{2}+\gamma_{2}+a_{3}+x_{3}+\beta_{3}+\gamma_{3}\right)} \\
& (-1)^{\left(x_{1}-i_{1}+\beta_{1}-j_{1}+x_{2}-i_{2}+\beta_{2}-j_{2}+x_{3}-i_{3}+\beta_{3}-j_{3}\right)} \\
& e^{-\lambda_{3} t\left(\frac{\theta_{3}}{t}+x_{3}-i_{3}+\beta_{3}-j_{3}+\gamma_{3}\right)} \\
& \overline{t\left(\frac{\left(\theta_{1}-\theta_{2}\right)}{t}+x_{1}-i_{1}+\beta_{1}-j_{1}+\gamma_{1}\right)} \\
& {\left[\frac{\left(1-e^{-\lambda_{3} t\left(\frac{\left(\theta_{2}-\theta_{3}\right)}{t}+x_{2}-i_{2}+\beta_{2}-j_{2}+\gamma_{2}\right)}\right)}{t\left(\frac{\left(\theta_{2}-\theta_{3}\right)}{t}+x_{2}-i_{2}+\beta_{2}-j_{2}+\gamma_{2}\right)}-\frac{\left(1-e^{-\lambda_{3} t\left(\frac{\left(\theta_{1}-\theta_{3}\right)}{t}+x_{1}-i_{1}+\beta_{1}-j_{1}+\gamma_{1}+x_{2}-i_{2}+\beta_{2}-j_{2}+\gamma_{2}\right)}\right)}{t\left(\frac{\left(\theta_{1}-\theta_{3}\right)}{t}+x_{1}-i_{1}+\beta_{1}-j_{1}+\gamma_{1}+x_{2}-i_{2}+\beta_{2}-j_{2}+\gamma_{2}\right)}\right]}
\end{aligned}
$$

Para que a expressão acima seja válida e não encontremos nenhum denominador igual a 0 , uma possibilidade é estabelecer $\theta_{3}>\theta_{2}>\theta_{1}$. 
APÊNDICE D 


\section{Referências Bibliográficas}

Barlow et al.(1990) Richard E. Barlow, Igor Bazovsky e Sérgio Wechsler. Classical and Bayes approaches to environmental stress screening (ESS): a comparision. Em Proceedings of Annual Reliability and Maintainability Symposium, University of California, Berkeley, páginas 81-84. Citado na pág. 1, 9

Barlow et al.(1994) Richard E. Barlow, Carlos A.B. Pereira e Sérgio Wechsler. A Bayesian approach to environmental stress screening. Naval Research Logistics, 41:215-228. Citado na pág. $1,9,10,11,12,18,39$

Beaumont(2010) Mark A. Beaumont. Approximate Bayesian computation in evolution and ecology. Annual Review of Ecology, Evolution and Systematics, 41:379-406. Citado na pág. 6

Beaumont et al.(2002) Mark A. Beaumont, Wenyang Zhang e David J. Balding. Approximate Bayesian computation in population genetics. Genetics, 162:2025-2035. Citado na pág. 5

Beaumont et al.(2009) Mark A. Beaumont, Jean-Marie Cornuet, Jean-Michel Marin e Christian P. Robert. Adaptative approximate Bayesian computation. Biometrika, asp052:1-8. Citado na pág. 6

Bonassi(2013) Fernando V. Bonassi. Approximate Bayesian computation for complex dynamic systems. Tese de Doutorado, Department of Statistical Science, Duke University. Citado na pág. 1, 4

Campos e Wechsler(2012) Thiago F. Campos e Sérgio Wechsler. ABC for kids. Em XI Brazilian Meeting on Bayesian Statistics: EBEB 2012, volume 1490, páginas 67-74. Citado na pág. 3

Devroye(1986) Luc Devroye. Non-Uniform Random Variate Generation. Springer-Verlag, primeira edição. Citado na pág. 27

Fearnhead e Prangle(2012) Paul Fearnhead e Dennis Prangle. Constructing summary statistics for approximate Bayesian computation: semi-automatic approximate Bayesian computation. Journal of the Royal Statistical Society, 74(3):419-474. Citado na pág. 6

Jasra et al.(2012) Ajay Jasra, Summetpal S. Singh, James S. Martin e Emma McCoy. Filtering via approximate Bayesian computation. Stat Comput, 22:1223-1237. Citado na pág. 7

Marin et al.(2011) Jean-Michael Marin, Pierre Pudlo, Christian P. Robert e Robin J. Ryder. Approximate Bayesian computational methods. Statistics and Computing, 21(2):289-291. Citado na pág. $1,3,4,5,6$

Marjoram et al.(2003) Paul Marjoram, John Molitor, Vincent Plagnol e Simon Tavaré. Markov chain Monte Carlo without likelihoods. Proceedings of the National Academy of Sciences, 100: 15324-15328. Citado na pág. 5

Perlstein et al.(1987) Howard J. Perlstein, John W. Littlefield e Igor Bazovsky. The quantification of environmental stress screening. Proceedings of the Institute of Environmental Sciences, páginas 202-208. Citado na pág. 9 
Pritchard et al.(1999) Jonathan K. Pritchard, Mark T. Seielstad, Anna Perez-Lezaun e Marcus W. Feldman. Population growth of human Y chromosomes: a study of Y chromosome microsatellites. Molecular Biology and Evolution, 16:1791-1798. Citado na pág. 4

Reddy e Dietrich(1994) Rajasekhar K. Reddy e Duane L. Dietrich. A 2-level environmentalstress-screening (ess) model: a mixed-distribution approach. IEEE Transactions on Reliability, 43:85-90. Citado na pág. 1

Rubin(1984) Donald B. Rubin. Bayesianly justifiable and relevant frequency calculations for the applied statistician. Annals of Statistics, 12(4):1151-1172. Citado na pág. 3

Sisson et al.(2007) Scott A. Sisson, Yanan Fan e Mark M. Tanaka. Sequential Monte Carlo without likelihoods. Proceedings of the National Academy of Sciences of the United States, 104:1760-1765. Citado na pág. 6

Tavaré et al.(1997) Simon Tavaré, David J. Balding, Robert C. Griffiths e Peter Donnely. Inferring coalescence times from DNA sequence data. Genetics, 145(2):505-518. Citado na pág. 4

Toni et al.(2009) Tina Toni, David Welch, Natalja Strelkowa, Andreas Ipsen e Michael P.H. Stumpf. Approximate Bayesian computation scheme for parameter inference and model selection in dynamical systems. Journal of the Royal Society Interface, 6:187-202. Citado na pág. 6

Turner e Van Zandt(2012) Brandon M. Turner e Trisha Van Zandt. A tutorial on approximate Bayesian computation. Journal of Mathematical Psychology, 56:69-85. Citado na pág. 5, 6

Wilkinson(2009) Richard D. Wilkinson. Approximate Bayesian computation (ABC) gives exact results under the assumption of model error. Tech. Rep. 0811.3355 arXiv.org, 2009. Citado na pág. 6

Yang(2002) Guangbin Yang. Environmental-stress-screening using degradation measurements. IEEE Transactions on Reliability, 51(3):288-293. Citado na pág. 9 